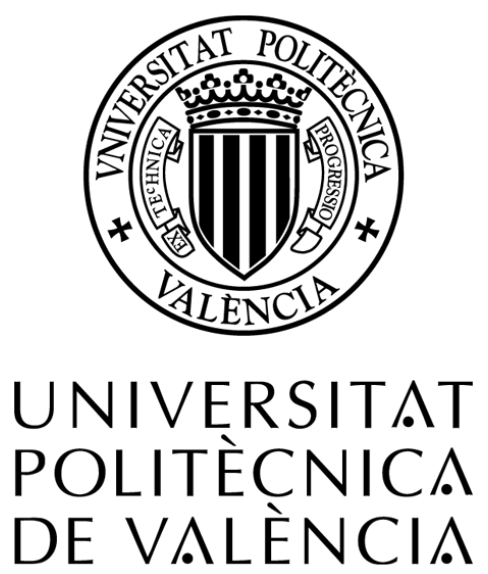

\title{
CONJUGACY CLASSES \\ AND \\ FACTORISED GROUPS
}

Author

Víctor Manuel Ortiz Sotomayor

\section{Supervisors}

Ph. D. María José Felipe Román

Ph. D. Ana Martínez Pastor

Valencia, May 2019 

Doña María José Felipe Román, Titular de Universidad de la Universitat Politècnica de València, y Doña Ana Martínez Pastor, Catedrática de Universidad de la Universitat Politècnica de València

\section{CERTIFICAN}

que la presente memoria "Conjugacy classes and factorised groups" ha sido realizada bajo nuestra dirección por Víctor Manuel Ortiz Sotomayor y constituye su tesis para optar al grado de Doctor en Matemáticas, con mención "Doctor Internacional”.

Y para que así conste en cumplimiento de la legislación vigente presentamos y apadrinamos ante la Escuela de Doctorado de la Universitat Politècnica de València la referida tesis firmando el presente certificado.

Valencia, mayo de 2019

Las directoras

María José Felipe Román

Ana Martínez Pastor 


\begin{abstract}
Adivina adivinanza
dime la ciudad que es

dos columnas

non plus ultra

su nombre empieza por $C$

tiene un pasado fenicio

y al mar abierto sus puertas

unos me dicen que es Cai

y otros me dicen que Ceuta.
\end{abstract}

- Comparsa "Los del ferry"

CARNAVAl DE CÁDIZ 2019 
$I^{2}$ have been some time thinking about the best language choice for this significant part of my thesis. Honestly, I believe that the main purpose of the acknowledgements is that they reach with clarity to the people they are intended. Therefore, I apologise to the reader for the discourtesy of expressing my gratitude mainly in my mother tongue, and also in Italian and Valencian.

First of all, I would like to acknowledge to all the external evaluators and members of my thesis committee, for the thorough reading of this dissertation and for all the recommendations which certainly improved it.

Respecto a los agradecimientos más personales, entre todas las personas que me han acompañado en este largo camino, me gustaría resaltar especialmente a dos de ellas, fuera de todo formalismo. Todo este trabajo hubiese sido imposible sin la inestimable ayuda de mis directoras de tesis, Ana y María José. En primer lugar, os doy las gracias por transmitirme con generosidad vuestros conocimientos en este área de las matemáticas, por vuestra infinita paciencia y por la confianza que siempre habéis depositado en mí desde el primer día que nos conocimos. Gracias por saber encontrar en vuestras agendas el tiempo y la dedicación que esta tarea requiere, por las cuidadosas lecturas de trabajos e informes, que no han sido pocos, y vuestras siempre significantes mejoras. Ha sido un placer poder aprender estos años de vuestro instinto investigador, vuestra experiencia y vuestra capacidad de trabajo. Pero no solo destacáis por vuestras representaciones, sino sobre todo por vuestros excepcionales caracteres. Habéis ejercido también especialmente como tutoras y en todo momento he estado orientado y motivado por vuestros consejos y experiencias personales. Al igual que el tema principal de esta tesis, habéis combinado dos personalidades diferentes, dando lugar a un tándem perfecto como codirectoras. Sé que no todo el mundo lo puede decir, pero me llevo una relación de amistad con ustedes. Teneros como directoras ha sido un regalo.

Vorrei esprimere la mia completa gratitudine al Professor Silvio Dolfi, per avermi accettato e inserito nel Suo gruppo ricercatore dell'Università di Firenze e per avermi fatto scoprire un'altra interessante area di ricerca. Senza dubbio è stato un autentico privilegio potere imparare da Lei e da tutto il Suo gruppo, dalla Vostra conoscenza, dal Vostro gran intuito nell'approccio ai problemi e dalla Vostra abilità e competenza. Ringrazio Lei e il dipartimento tutto per avermi dimostrato in ogni momento vicinanza e affetto, che senza dubbio hanno reso la mia permanenza lì un dono. Grazie Emanuele Pacifici, per avermi invitato a conoscere l'Università di Milano e i suoi componenti. Gracias especialmente a Lucía Sanus, la mejor guía de Florencia y de parte de Italia, por ayudarme durante mis estancias en innumerables aspectos cotidianos e investigadores.

Me gustaría darles las gracias a los miembros del Instituto Universitario de Matemática Pura y Aplicada de la Universitat Politècnica de València por proporcionarme un sitio de trabajo y estudio relajado y con todas las necesidades cubiertas. En particular, agradecer a todo el equipo investigador de álgebra la calurosa acogida que me brindaron, tanto los miembros UPV como 
los pertenecientes a la Universitat de València y la Universitat Jaume I de Castelló. Gracias Félix Martínez y Minerva Báguena por los incontables trámites con los que me habéis ayudado. En especial, gracias Pepe Bonet por tu trato siempre amable y cercano y por tu apoyo inicial cuando no tenía financiación. Muchas gracias a todos los profesores del Departamento de Matemática Aplicada de la Escuela de Informática, sobre todo a Llanos Gascón y Paco Monserrat, por facilitarme mi etapa docente en dicha escuela.

Gràcies Xaro Soler per, segurament sense saber-ho, generar en mi un gust especial per l'àlgebra i especialment per la teoria de grups al llarg del Grau en Matemàtiques en la Universitat d'Alacant. Sense dubte, tens el do de transmetre la teua passió pels grups. Ser el teu alumne i veure't donar classe ha sigut un plaer. Gràcies pels teus consells i conversacions que seguim compartint des de llavors.

Aprovecho para dedicarles estas líneas a mi familia y en especial a mis padres, por la libertad que siempre me habéis brindado a la hora de elegir mi camino académico. Sin duda, en este proyecto ha resultado fundamental la constancia, la perseverancia y el sacrificio que siempre me inculcasteis. Agradeceros el apoyo, el cariño y la educación que me habéis dado. Os doy las gracias por sacrificar muchos momentos buenos de vuestras vidas, para poder después disfrutarlos en los míos. No hay un manual de cómo ejercer de madre y de padre, pero sinceramente creo que lo habéis hecho de maravilla. Os quiero.

Echo la vista atrás y recuerdo que llegué a esta parte de España sin conocer prácticamente a nadie. Sin embargo, estos años he tenido la oportunidad de relacionarme con muchas personas de diferentes lugares que me han acompañado durante este camino. Agradeceros Mario y Joselu los innumerables buenos momentos vividos desde la carrera y vuestra entrañable amistad iluminada por la sonrisa de Fer, quien siempre estará con nosotros. Gracias Pablo y Cristina, mis compañeros de viaje, por ser mis pilares de confianza incluso desde la distancia. Grazie mille Spagnuolo per i tuoi sempre utili consigli e per le eccezionali traduzioni. Gràcies Vicent per les xarrades en valencià que tantíssim em van ajudar. Agradecerte Villacampa tus psicológicas conversaciones tan necesarias siempre. Especialmente, gracias a ti Laura por tu calma, por hacerlo todo más sencillo y por compartir conmigo en estos últimos años tantos momentos felices. Agradecerte también el diseño paciente y cariñoso de la cubierta de esta tesis. Sería imposible hacer una lista completa de todos, pero espero que os sintáis igualmente reflejados en estas palabras.

En el año 2010 dejé mi ciudad natal para realizar mis estudios universitarios, siempre a la vera del Mediterráneo. Aunque esta comunidad autónoma me ha ofrecido muchas oportunidades, cada día siento más nostalgia de mi Ceuta, mi familia, mis amistades... y es que uno siempre es de donde ha nacido. Gracias a mis amigos caballas por los buenos momentos en todos estos años. También agradezco a Ceuta las oportunidades y la formación académica que me proporcionó, la tolerancia cultural que me enseñó y la personalidad andaluza que me inculcó. Aprovecho para invitar a todo aquel que lea estas letras a visitar esta gran desconocida parte de España, la "perla del Mediterráneo".

Valencia, mayo de 2019

Víctor M. Ortiz Sotomayor 
This dissertation has been elaborated at the Instituto Universitario de Matemática Pura y Aplicada de la Universitat Politècnica de València (IUMPA-UPV), thanks mainly to the financial support of the predoctoral grant ACIF/2016/170 from Generalitat Valenciana (Spain). The first academic year was supported by Proyecto Prometeo II/2013/013 from Generalitat Valenciana. The institute IUMPA has financed some travel expenses of the author's attendances to research conferences. This research has been partially supported by Proyecto PGC2018-096872-B-I00, Ministerio de Ciencia, Innovación y Universidades.

The mobility grant BEFPI/2018/025 from Generalitat Valenciana has allowed the author to perform a research stay of three months (March-May 2018) at the Dipartimento di Matematica e Informatica "U. Dini" (DIMAI) of Università di Firenze (Italy). The author has also been granted with a Borsa Ferran Sunyer i Balaguer for a research stay at Università di Firenze in April 2019. 



\section{List of included articles}

This dissertation is a compendium of research publications. It consists of an introductory part and the following manuscripts:

[A] M. J. Felipe, A. Martínez-Pastor, and V. M. Ortiz-Sotomayor. "Square-free class sizes in products of groups". J. Algebra 491 (2017) 190-206.

[B] M. J. Felipe, A. Martínez-Pastor, and V. M. Ortiz-Sotomayor. "Prime power indices in factorised groups". Mediterr. J. Math. 14.6 (2017) article: 225.

[C] M. J. Felipe, A. Martínez-Pastor, and V. M. Ortiz-Sotomayor. "Zeros of irreducible characters in factorised groups". Ann. Mat. Pura Appl. 198 (2019) 129-142.

[D] M. J. Felipe, A. Martínez-Pastor, and V. M. Ortiz-Sotomayor. "Products of groups and class sizes of $\pi$-elements". Accepted up to final revision in Mediterr. J. Math. (2019).

All the above articles are either published or accepted in journals included in the Journal Citation Reports (JCR). In particular, $[\mathrm{D}]$ is currently accepted up to final revision in Mediterranean Journal of Mathematics. The versions presented here are adaptations for the thesis due to university regulations. Each of them appears as an appendix having exactly the same structure and bibliography as the original published version. 



\section{Contents}

Acknowledgements v v

\begin{tabular}{ll}
\hline List of included articles & ix
\end{tabular}

Summary xiii

$\begin{array}{lll}1 & \text { Introduction } & 1\end{array}$

2 General discussion of the results 5

$2.1 \quad$ Square-free and prime power class sizes $\ldots \ldots \ldots \ldots \ldots$

2.2 Zeros of irreducible characters and $\pi$-elements . . . . . . . . . . . . . . 8

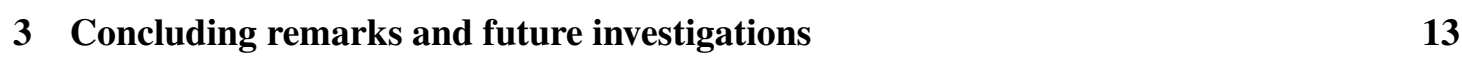

\begin{tabular}{ll}
\hline Bibliography & 17
\end{tabular}

\begin{tabular}{|l|l|}
\hline A Square-free class sizes in products of groups & 19
\end{tabular}

\begin{tabular}{|l|}
\hline B Prime power indices in factorised groups \\
\hline
\end{tabular}

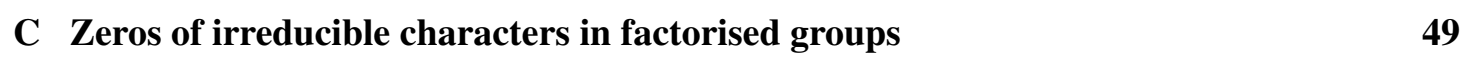

\begin{tabular}{|l|l|l|l|l|l}
\hline Products of groups and class sizes of $\pi$-elements & 65
\end{tabular} 



\title{
Summary
}

\author{
A person who is tired of group theory is a person who \\ is tired of life.
}

- SidNey COLEMAN

\section{I | English}

$\mathrm{T}$

he influence of the conjugacy class sizes on the structure of a group has been a widely investigated problem within finite group theory. In the last decades, several researchers have obtained new progress in this direction. Specially, some relevant information is provided by the class sizes of certain subsets of elements of the group, as prime power order elements, $p$-regular elements, etc. Other subsets of elements that have recently attracted interest are defined via the character table of the group, as vanishing elements and real elements.

In parallel to this research on conjugacy classes, the study of groups which can be factorised as a product of two subgroups has gained increasing interest. In particular, the structure of factorised groups such that different families of subgroups of the factors satisfy certain permutability conditions has recently been analysed.

In this thesis we aim to combine in a novel way both perspectives of group theory. In this framework of very scarce literature, our main purpose is to obtain new contributions about the global structure of a factorised group when the class lengths of some elements in its factors verify certain arithmetical properties.

Square-free class length conditions on ( $p$-regular) prime power order elements are considered for products of two subgroups, occasionally mutually permutable. Prime power class sizes are investigated for arbitrary products of two groups, avoiding the use of permutability conditions between the factors. The concept of a core-factorisation of a group, which particularly extends products of mutually permutable subgroups, is introduced for the first time in this dissertation, and it has been revealed determinant within this context. Precisely, this notion emerges when discussing the above arithmetical properties on the class sizes of vanishing elements, interplaying as a novelty character theory and the research on factorised groups. Core-factorisations are also exploited when analysing $\pi$-number and $\pi^{\prime}$-number class lengths for (prime power order) $\pi$-elements in the factors of a factorised group.

\section{II | Spanish}

Ч ${ }^{\mathrm{n}}$ problema clásico en la teoría de grupos finitos es el estudio de cómo los tamaños de las clases de conjugación influyen sobre la estructura del grupo. En las últimas décadas, numerosos investigadores han obtenido nuevos avances en esta línea. Especialmente, se han probado resultados interesantes a partir de la información proporcionada por los tamaños de clase de algún subconjunto de elementos del 
grupo, como los elementos de orden potencia de primo, elementos $p$-regulares, etc. Además, ciertos subconjuntos de elementos definidos a través de la tabla de caracteres del grupo están siendo investigados recientemente, como los elementos anuladores y los elementos reales.

Por otra parte, en los últimos años, el estudio de grupos factorizados como producto de subgrupos ha sido objeto de creciente interés. En particular, diversos autores han analizado la estructura de grupos factorizados en los que diferentes familias de subgrupos de los factores satisfacen ciertas condiciones de permutabilidad.

En esta tesis pretendemos conjugar ambas perspectivas de actualidad en la teoría de grupos de manera novedosa. Así, en este contexto de literatura escasa, el objetivo es obtener nuevas contribuciones acerca de la estructura global de un grupo factorizado a partir de ciertas propiedades aritméticas de los tamaños de las clases de algunos elementos de sus factores.

Estudiamos productos de dos subgrupos, eventualmente mutuamente permutables, donde los elementos ( $p$-regulares) de orden potencia de primo de los factores tienen tamaños de clase libres de cuadrados. Analizamos el caso de tamaños de clase potencias de primos para grupos factorizados arbitrarios, evitando el uso de condiciones de permutabilidad entre los factores involucrados. El concepto de una core-factorización de un grupo, que extiende en particular a los productos mutuamente permutables, es introducido por primera vez en esta tesis y ha resultado crucial dentro de este contexto. Esta noción surge precisamente cuando consideramos las anteriores propiedades aritméticas para los tamaños de clase de elementos anuladores, interrelacionando novedosamente la teoría de caracteres con la investigación en grupos factorizados. Finalmente, estudiamos grupos que poseen una core-factorización cuyos tamaños de clase de $\pi$-elementos (de orden potencia de primo) son $\pi$-números o $\pi^{\prime}$-números.

\section{Valencian}

T n problema clàssic dins de la teoria de grups finits és l'estudi de com els tamanys de les classes de conjugació influeixen sobre l'estructura del grup. En les últimes dècades, nombrosos investigadors han obtingut nous avanços en aquesta línia. Especialment, s'han provat resultats interessants a partir de la informació proporcionada pels tamanys de classe d'algun subconjunt d'elements del grup, com els elements d'ordre potència de primer, elements $p$-regulars, etc. A més, certs subconjunts d'elements definits a través de la taula de caràcters del grup estan sent investigats recentment, com els elements anul-ladors i els elements reals.

D'altra banda, en els últims anys, l'estudi de grups factoritzats com a producte de subgrups ha sigut objecte de creixent interés. En particular, diversos autors han analitzat l'estructura de grups factoritzats en els quals diferents famílies de subgrups dels factors satisfan certes condicions de permutabilitat.

En aquesta tesi pretenem conjugar ambdues perspectives d'actualitat en la teoria de grups de manera innovadora. En aquest context de literatura escassa, l'objectiu és obtenir noves contribucions sobre l'estructura global d'un grup factoritzat a partir de certes propietats aritmètiques dels tamanys de les classes d'alguns elements dels seus factors.

Estudiem productes de dos subgrups, eventualment mútuament permutables, on els elements $(p$ regulars) d'ordre potència de primer dels factors tenen tamany de classe llibre de quadrats. Analitzem el cas de tamanys de classe potències de primers per a grups factoritzats arbitraris, evitant l'ús de condicions de permutabilitat entre els factors involucrats. El concepte d'una core-factorització d'un grup, que estén particularment als productes mútuament permutables, és introduït per primera vegada en aquesta tesi $\mathrm{i}$ ha resultat determinant dins d'aquest context. Aquesta noció sorgeix precisament quan considerem les propietats aritmètiques anteriors per als tamanys de classe d'elements anul-ladors, interrelacionant innovadorament la teoria de caràcters amb la investigació en grups factoritzats. Finalment, estudiem grups els quals posseeixen una core-factorització on els tamanys de classe dels $\pi$-elements (d'ordre potència de primer) són $\pi$-números o $\pi^{\prime}$-números. 


\title{
Introduction
}

\author{
Innovation is taking two things that already exist \\ and putting them together in a new way.
}

- TOM FRESTON

$\mathrm{T}$ he main topic of this thesis is framed within finite group theory. Groups are a basic algebraic structure, which turn out to be a significant tool in the analysis of different situations where symmetries are involved. Within mathematics itself, groups have an instrumental character, since numerous mathematical structures can be studied from the point of view of groups that act on them. Furthermore, there are innumerable applications of this algebraic theory in diverse scientific and technical areas: Informatics and Telecommunications (automata theory and languages, coding theory, cryptography, ...), Physics, Chemistry, Biology, Economy, Architecture, and even Fine Arts.

The research developed in this dissertation aims to contribute to the knowledge of the internal structure of a finite group, from the analysis of its arithmetical properties and the relationship between its subgroups and the group itself. More concretely, the thesis combines two wellestablished research lines in finite group theory: the influence of the conjugacy class sizes on the group structure and the study of groups which can be written as a product of subgroups.

During the last decades, the connection between the structure of a group and certain sets of positive integers which are naturally associated to it has been very widely investigated, and it continues being an active line of research. One of the most classical ones is the set of the conjugacy class sizes. This subject is closely related to the research on representation and character theory, which is currently a significant area of investigation. Indeed, several problems on conjugacy class sizes have also been analysed from the character perspective, and they surprisingly behave often in a dual way. Besides, some results in this context require for their achievement the use of representation and character theory. We can mention as an example the celebrated Burnside's $p^{\alpha}$-lemma about the non-simplicity of groups with a conjugacy class of prime power size.

Moreover, instead of considering the full set of conjugacy class sizes, several researchers have also examined whether some subsets of it are enough in order to provide structural criteria. For instance, an important role has been played by prime power order elements, $p$-regular elements for a given prime $p$, vanishing elements or real elements. This last approach involves a greater difficulty, since some information about the group is partially lost, and some results derived from the classification of finite simple groups (CFSG) are often needed. 
Within this research line, in 1953 Baer characterised finite groups such that every prime power order element has prime power class size $([\overline{\mathbf{6}}])$. Chillag and Herzog $([\mathbf{1 6}])$ proved in 1990 that a given prime $p$ does not divide any class size of a group $G$ if and only if $G$ has a Sylow $p$-subgroup in its centre. Later on, Liu, Wang and Wei showed in [31] that a group has a Sylow $p$-subgroup as a direct factor if and only if all the class sizes of $p$-regular prime power order elements are not divisible by $p$, for which they made use of the CFSG through a known result about transitive actions of permutation groups. This arithmetical condition on the class sizes has also been discussed for vanishing elements in [14], proving that such groups are $p$-nilpotent. Recently, the problem about the existence of nilpotent and abelian Hall $\pi$-subgroups, for a set of primes $\pi$, has been characterised via the set of class sizes in [10]. Several international research groups, among them the one of the supervisor Felipe, have deeply discussed this issue. The exhaustive report [15] due to Camina and Camina describes a general perspective about the subject until 2011, and the recent case of vanishing elements is treated in [22] by Dolfi, Pacifici and Sanus.

In parallel with the previous developments, the research on groups which can be factorised as a product of subgroups has become increasingly relevant, in the universe of finite groups as well as in the infinite one. In this line, a good number of authors have carried out in-depth investigations with the purpose of understanding how some information about the subgroups that appear in the factorisation affects the whole group structure. We refer the interested reader to the book [2] which gathers excellently this progress, specially in the infinite case.

The origin of this strand of research is remote, and it could be traced back to 1903, when Burnside proved the aforementioned result which can be rephrased as follows: a group factorised as a product of a Sylow subgroup and the centraliser of a non-trivial element cannot be simple. Thirty years later, Hall laid the foundations for fruitful developments on the theory of soluble groups, and in particular he proved that a finite soluble group is characterised by being the product of pairwise permutable Sylow subgroups. The next milestone in this context is the elementary proof due to Itô in the 50's of the following fact: a (not necessarily finite) group is metabelian whenever it is the product of two abelian groups. All these results inspired the conjecture about the solubility of a finite group which is the product of two nilpotent subgroups. That conjecture was proved in the positive way by Wielandt in 1958 for the case of factors of relatively prime orders, and later by Kegel in the general case. Indeed, Fitting had previously demonstrated that the product of two normal nilpotent subgroups is nilpotent. In contrast to this result, it is well-known that a finite group does not need to be supersoluble whenever it is the product of two normal supersoluble subgroups, although central and direct products of supersoluble subgroups so are. This last fact motivated the inquiry of new permutability properties between the factors, stronger than normality, aiming to obtain supersolubility criteria for products of supersoluble subgroups.

The starting point of these investigations could be located in the paper [5] due to Asaad and Shaalan. These authors proved, among other results, that a product $G=A B$ of supersoluble subgroups $A$ and $B$ is supersoluble if either every subgroup of $A$ permutes with every subgroup of $B$, or $A$ permutes with every subgroup of $B, B$ permutes with every subgroup of $A$, and $G^{\prime}$ is nilpotent. Subgroups $A$ and $B$ satisfying the latter conditions are called totally and mutually permutable, respectively, and products of such subgroups somehow form extensions of central and normal products. In relation to the first permutability property, the notion of total (complete) conditional permutability is studied in $[3,4,27]$. These concepts are still under active research, as shown by the vast bibliography on the subject with contributions from several international research groups, in particular that of the supervisor Martínez-Pastor. The book $[\mathbf{8}]$ surveys excellently the most significant achievements in this framework until 2010.

A new research area arises when both current perspectives are joined, i.e. the analysis of the 
structure of a factorised group via the conjugacy class sizes in the whole group of the elements in its factors. The pioneering paper within the scarce literature in this line could be situated in [31], where the authors proved that if a group is the product of two subgroups that permute with any other subgroup, and all the elements in the factors have square-free class sizes, then it is supersoluble. This publication is the motivation of [7] due to Ballester-Bolinches, Cossey and $\mathrm{Li}$, where analogous problems are examined for products of mutually permutable subgroups. A natural path of research opens up by considering other arithmetical conditions on the class sizes of the elements in the factors of a factorised group, as well as different families of elements lying in that factors.

This current and incipient study that combines both perspectives is expected to be a useful tool in order to get a better knowledge about the structure of finite groups. In this spirit, the contributions of this thesis represent a considerable advance in this research line. This development provides further evidence that in mathematics, and in particular in group theory, different areas are not in watertight compartments. Not only the research lines of factorised groups and conjugacy class sizes are interwoven in this dissertation, but also character theory and products of groups are joined in $[\bar{C}]$. Besides, this investigation introduces a new way of filtering the set of all the conjugacy class sizes of a group with the purpose of obtaining structural criteria for it.

In all the papers presented in this dissertation we have focused on the case of a group $G$ which is the product of two subgroups $A$ and $B$, so $G=A B$, as this is the first natural case to explore. A key fact frequently used in this framework is the following one: in a factorised group $G=A B$, for any prime $p$ there exists a Sylow $p$-subgroup $P$ of $G$ such that $P=(P \cap A)(P \cap B)$ being $P \cap A$ and $P \cap B$ Sylow $p$-subgroups of $A$ and $B$, respectively. This result, which is a slight reformulation of [2, Corollary 1.3.3], allows us to obtain a Sylow subgroup which somehow inherits the factorisation of the whole group. This also holds for Hall $\pi$-subgroups when the group is $\pi$-separable, and for products of more than two subgroups whenever they are pairwise mutually permutable ([8 . Proposition 4.1.45]). Nevertheless, we have aimed to avoid, as far as possible, the use of conditions of permutability between the subgroups appearing in the factorisation. In fact, one of the central open questions in this research area is whether it is possible to remove, or at least to weaken, the permutability hypotheses on the factors in the existing results. In this sense, in $[\mathrm{B}]$ we have not used any permutability condition on the factors; and in $[\bar{C}]$ we have introduced one of the main concepts of this thesis, core-factorisations of a group, which is a much weaker assumption than having products of mutually permutable subgroups. As we shall see, factorisations of this kind naturally arise when dealing with minimal normal subgroups contained in one of the factors, and they are characterised by the existence of a chief series related to the factorisation of the group (see [C, Lemma 2]).

It is a fact of common knowledge that (normal) subgroups of factorised groups might not inherit the factorisation of the whole group, which emphasises even more the relevance of the aforementioned result about Sylow subgroups. If we focus now on the framework of conjugacy classes, we remark that the information about class sizes of elements which are product of one element in each factor is lost in this factorised-group context. Furthermore, it is widely known that there is no relation a priori between the primes that divide the class sizes of the elements in a subgroup and the primes that divide the corresponding class sizes in the whole group. This fact certainly happens in our case for a factorised group and its factors. However, as it will be shown, we have been able to obtain some surprising connection between these primes in special situations.

In the following chapter a more detailed description of our main achievements is discussed. In the first section we concentrate on square-free and prime power class sizes of ( $p$-regular) prime power order elements in products of two subgroups, occasionally mutually permutable. In the second section we consider other families of elements as vanishing elements and $\pi$-elements, 
in the context of core-factorisations of groups. Finally, some conclusions and open questions about the research developed are treated in the last chapter. All the results in this thesis appear in the manuscripts listed at the beginning, which are attached as appendices in chronological order at the final part of this dissertation. Although each publication has its own notation and terminology explained in its introduction, hereafter we unify it for comprehensiveness. In the sequel, all groups under consideration are finite, and $G$ will denote a group. For an element $x \in G$ the conjugacy class of $x$ in $G$, i.e. the set of all conjugates to $x$ in $G$, is denoted by $x^{G}$, and its size is $\left|x^{G}\right|=\left|G: \mathbf{C}_{G}(x)\right|$. A $p$-regular element is such an element whose order is not divisible by $p$, where $p$ will always be a prime number. We represent the set of all prime divisors of a natural number $n$ by $\pi(n)$. As customary, the set of all Sylow $p$-subgroups of $G$ is expressed by $\operatorname{Syl}_{p}(G)$, whilst $\operatorname{Hall}_{\pi}(G)$ is the set of all Hall $\pi$-subgroups of $G$ for a set of primes $\pi$. A group such that $G=\mathbf{O}_{\pi}(G) \times \mathbf{O}_{\pi^{\prime}}(G)$ is said to be $\pi$-decomposable. Given a group $G=A B$ which is the product of the subgroups $A$ and $B$, a subgroup $S$ is called prefactorised (with respect to this factorisation) if $S=(S \cap A)(S \cap B)$. As usual, CFSG will denote the classification of finite simple groups. The remaining notation and terminology is standard within the theory of finite groups and we mainly follow the book [19]. 


\section{General discussion of the results}

Groups, as people, will be known by their actions.

- Guillermo Moreno

\subsection{Square-free and prime power class sizes}

$\mathrm{T}$ his section summarises the main results of the manuscripts $[\mathrm{A}]$ and $[\mathrm{B}]$. As previously said, the background of this strand of research concerning conjugacy class sizes and factorised groups is limited. Its origin could be traced back to the paper [31] of Liu, Wang and Wei in 2005. These authors initially studied products $G=A B$ of two normal subgroups such that their prime power order elements in $A \cup B$ have square-free class sizes in $G$, and they proved that such groups are supersoluble (cf. [31, Proposition 9]). Indeed, they also obtained the same thesis under the weaker assumption that $A$ and $B$ permute with every subgroup of $G$ (i.e. $A$ and $B$ are permutable in $G$ ), but strengthening the square-free class size hypothesis to all the elements in $A \cup B$, instead of considering only the prime power order ones ([31. Theorem 10]). Nonetheless, both impositions on the factors are strong since, for instance, $A$ and $B$ inherit the class size hypotheses. This is due to the fact that they are subnormal in $G$, so by elementary class size properties it holds that $\left|x^{A}\right|$ divides $\left|x^{G}\right|$ for any $x \in A$ (and the same happens for $B$ ).

The next contribution in this area did not appear until the year 2013 with the paper [7] due to Ballester-Bolinches, Cossey and Li. A first purpose was to relax the above permutability hypotheses and, in this scene, products of mutually permutable subgroups naturally emerge. We recall that two subgroups $A$ and $B$ are mutually permutable if $A$ permutes with every subgroup of $B$ and $B$ permutes with every subgroup of $A$ (that is, $H A=A H$ for all subgroups $H$ of $B$ and $K B=B K$ for all subgroups $K$ of $A$ ). These researchers obtained another supersolubility criterion for a product $G=A B$. More precisely, they proved that if $A$ and $B$ are mutually permutable and $p^{2}$ does not divide $\left|x^{G}\right|$ for every prime $p$ and every $p$-regular element $x \in A \cup B$, then $G$ is supersoluble. Observe that, if $A$ and $B$ are either normal or permutable in $G$, then they are certainly mutually permutable. Thus, the above cited Theorem 10 of [31] can be regarded as a particular case of this last result. Besides, they also handled products of pairwise mutually permutable subgroups (for more than two factors) whose elements in the factors have class lengths not divisible by a fixed prime $p$.

Groups all of whose conjugacy classes have square-free sizes were originally examined by Chillag and Herzog in 1990, and later on by Cossey and Wang (cf. [16, Theorem 1] and [18. 
Theorem 2], respectively). They not only proved the supersolubility of $G$, but also provided extra structural information on $G / \mathbf{F}(G)$ and $G^{\prime}$. Notice that if we consider the trivial factorisation $G=A=B$ in the aforementioned results for factorised groups, then the supersolubility of the group is obtained. Consequently, it is natural to wonder whether some features of $G / \mathbf{F}(G)$ and $G^{\prime}$ are still valid in the factorised-group context. In this direction, and by means of certain properties of mutually permutable products, we demonstrate in $[\mathrm{A}]$ the next result.

Theorem 2.1.1. ([A, Corollary $\mathrm{G}])$ Let $G=A B$ be the product of the mutually permutable subgroups $A$ and $B$. Suppose that $\left|x^{G}\right|$ is square-free for every $x \in A \cup B$. Then we have:

(1) $G$ is supersoluble.

(2) $G^{\prime}$ is abelian.

(3) $G / \mathbf{F}(G)$ and $G^{\prime}$ have elementary abelian Sylow subgroups.

(4) $G / \mathbf{F}(G)$ and $\mathbf{F}(G)^{\prime}$ have Sylow p-subgroups of order at most $p^{2}$, for each prime $p$.

We also prove in $[\mathrm{A}]$ that some of the above statements remain true when only prime power order elements (or $p$-regular elements) in the factors are considered, and we provide examples which show the scope of the hypotheses in each case. As a consequence, the above cited results of [7] and [31] now appear as corollaries.

Cossey and Wang also localised in [18. Theorem 1] this type of assumptions for a fixed prime $p$, that is, they considered groups such that all their conjugacy class sizes are not divisible by $p^{2}$. Following this philosophy, in 2014, Qian and Wang ([33]) went a step further by considering just conjugacy class sizes of $p$-regular elements of prime power order. As probably expected, they made use of the CFSG, through the next strong result in [24]: each transitive permutation group has a fixed-point free element of prime power order. Motivated by their results, we handle in [A] this situation for products of groups.

Theorem 2.1.2. ([A, Theorem $\mathrm{B}])$ Let $G=A B$ be the product of the mutually permutable subgroups $A$ and $B$, and let $p$ be a prime such that $\operatorname{gcd}(p-1,|G|)=1$. If $p^{2}$ does not divide $\left|x^{G}\right|$ for every $p$-regular element $x \in A \cup B$ of prime power order, then:

(1) $G$ is soluble.

(2) $G$ is p-nilpotent.

(3) The Sylow p-subgroups of $G / \mathbf{O}_{p}(G)$ are elementary abelian.

The initial result of Cossey and Wang, which involved all the class sizes of a group $G$, actually supplied more information about $G$. More concretely, it affirmed that " $P$ ' has order $p$ for a Sylow $p$-subgroup $P$ of $G$ " and " $G / \mathbf{O}_{p}(G)$ has Sylow $p$-subgroups of order at most $p$ ". Regarding this last claim, we have found an error in its proof. In fact, we present in $[\mathrm{A}]$ a counterexample suggested by Cossey himself, and checked with GAP $([\overline{34}])$, which shows that the claim is not true. Concerning the other assertion, we extend it whenever $p$-elements in $A \cup B$ are also considered in Theorem 2.1.2. Indeed, we prove a result for factorised $p$-groups.

Theorem 2.1.3. ([A, Theorem A]) Let $p$ be a prime number and let $P=A B$ be a $p$-group such that $p^{2}$ does not divide $\left|x^{P}\right|$ for all $x \in A \cup B$. Then $P^{\prime} \leqslant \mathbf{\Phi}(P) \leqslant \mathbf{Z}(P)$ and $P^{\prime}$ is elementary abelian of order at most $p^{2}$.

We point out that no permutability condition on the factors is imposed in this case. This result generalises for products of groups the well-known Knoche's theorem (see [30]). However, and in contrast to this result, we show in [A] that the converse of Theorem 2.1.3 does not hold.

Besides, in Theorem $\mathrm{C}$ of $[\overline{\mathrm{A}}]$ we extend a $p$-supersolubility criterion obtained in [7], by imposing the class size hypothesis only on $p$-regular elements of prime power order. 
It is worth remarking that, in products of mutually permutable subgroups, the conditions imposed on the conjugacy class sizes are not generally acquired by the subgroups in the factorisation (cf. [A, Remark 1(b)]). This significantly differs from what happens in products of two normal (or permutable) subgroups, as handled in [31].

The development discussed so far, together with a collection of classical results on squarefree class sizes, is gathered in the expository paper [25] which corresponds to the Proceedings of the Ischia Group Theory international conference in 2016.

Within this setting of products of groups and conjugacy class sizes, we also addressed the analysis of other arithmetical properties on the class lengths. In this regard, our next contributions are motivated by the mentioned Baer's characterisation in $[\mathbf{6}]$ concerning groups in which all the conjugacy classes of prime power order elements have prime power lengths. This result affirms that such groups have the structure $G=G_{1} \times \cdots \times G_{k}$ where the direct factors have pairwise relatively prime orders, and if $G_{i}$ is not of prime power order, then its order is divisible by two primes and its Sylow subgroups are abelian. Some decades later, Camina and Camina provided in [15] an alternative shorter proof of Baer's theorem, by means of analysing groups all of whose $p$-elements have prime power class sizes, for a given prime $p$. Accordingly to the terminology used by these authors in [15], such a group is called a $p$-Baer group, and a Baer group is analogously defined as a group such that every prime power order element has prime power class size. Following this terminology, we introduce in $[\bar{B}]$ the next concepts for a factorised group.

Definition 2.1.4. Let $G=A B$ be the product of the subgroups $A$ and $B$, and let $p$ be a prime.

- $G=A B$ is a $p$-Baer factorisation if $\left|x^{G}\right|$ is a prime power for every $p$-element $x \in A \cup B$.

- $G=A B$ is a Baer factorisation if $\left|x^{G}\right|$ is a prime power for every prime power order element $x \in A \cup B$, i.e. if it is a $p$-Baer factorisation for all $p$.

It is not difficult to find examples of such factorisations. As a sample, any product $G=A B$ of two ( $p$-)Baer groups $A$ and $B$ provides a ( $p$-)Baer factorisation whenever the commutator subgroup $[A, B]$ is trivial (this is called a central product).

Based on the research carried out in [6] and [15], we study the structural properties of a group which possesses a ( $p$-)Baer factorisation. First, we show in $[\mathrm{B}$, Theorem A], among other facts, that groups $G$ with a $p$-Baer factorisation are $p$-soluble of $p$-length at most $1, G / \mathbf{F}(G)$ has a unique abelian Sylow $p$-subgroup, and $G / \mathbf{C}_{G}\left(\mathbf{O}_{p}(G)\right)$ is $p$-decomposable. A key result used in its proof is due to Camina and Camina: any element of a group $G$ with prime power conjugacy class length lie in $\mathbf{F}_{2}(G)$, the second term of the Fitting series of $G$ (cf. [15. Theorem 1]). Further, we get additional information about the primes appearing as class sizes of the $p$-elements in the factors.

Theorem 2.1.5. ([B, Theorem $\mathrm{B}])$ Let $G=A B$ be a $p$-Baer factorisation, and let $P \in \operatorname{Syl}_{p}(G)$. Then there exist unique primes $q$ and $r$ such that $\left|x^{G}\right|$ is a q-number for every p-element $x \in A$, and $\left|y^{G}\right|$ is an $r$-number for every $p$-element $y \in B$, respectively. (Possibly $p \in\{q, r\}$ or $q=r$.) Moreover, $P \leqslant \mathbf{C}_{G}\left(\mathbf{O}_{\{q, r\}^{\prime}}(\mathbf{F}(G))\right)$, and $P \mathbf{O}_{q}(G) \mathbf{O}_{r}(G)$ is normal in $G$. Further:

(1) If $q=r=p$, then $G$ is $p$-decomposable.

(2) If $p \notin\{q, r\}$, then $P$ is abelian.

This relevant theorem allows us to retrieve the main result of [15] regarding $p$-Baer groups, and consequently it provides a new proof of Baer's characterisation (see $[\mathrm{B}$, Theorem 1]). We point out that a crucial fact for proving Theorem 2.1.5 is Lemma 4 of [11] (which is [B, Lemma 4]), that is of interest in its own right. In that paper, Berkovich and Kazarin also studied several problems involving prime power class sizes in finite groups. 
Afterwards, we consider all the prime divisors of the order of $G$, that is, we examine groups $G$ with a Baer factorisation. We show in Corollary C of $[\overline{\mathrm{B}}]$ that such groups verify that $G / \mathbf{F}(G)$ is abelian, and if $\mathbf{F}(G)$ is abelian, then $G$ is an A-group (that is, all the Sylow subgroups are abelian). Nonetheless, one of the most significant results in this direction is that there is a close relationship between the primes that divide the class lengths in a factor and in the whole group.

Theorem 2.1.6. ([ $[\mathrm{B}$, Proposition $\mathrm{D}])$ Let $G=A B$ be a Baer factorisation, and let $X \in\{A, B\}$. If $x \in X$ is a prime power order element, then $\left|x^{X}\right|$ divides $\left|x^{G}\right|$. In particular, $A$ and $B$ are Baer groups.

Thus the structure of $A$ and $B$ in a Baer factorisation $G=A B$ is well-known. Once more, in $[\bar{B}]$ we frequently insist in examples which show the scope of the hypotheses handled. For instance, the analogous conclusion to Theorem 2.1.6 fails in general for a $p$-Baer factorisation, since it may happen that $\left|x^{X}\right|$ and $\left|x^{G}\right|$ are relatively prime for $X \in\{A, B\}$.

Example 2.1.7. ([B, Final examples (2)]) Let $G$ be the semidirect product of a non-abelian group of order 21 acting on an elementary abelian group of order 8 , in such a way that the subgroup of order 7 permutes the involutions transitively. Then there is a subgroup $A$ of order 24 and a subgroup $B$ of order 7 such that $G=A B$ is a 2 -Baer factorisation. Observe that every 2-element in $A \cup B$ has conjugacy class size in $G$ equal to 7 , but there exists a 2-element $x \in A$ such that $\left|x^{A}\right|=3$.

Furthermore, in contrast to Baer groups, a Baer factorisation might not be the direct product of Hall subgroups for disjoint sets of primes. Even so, we have attained an arithmetical characterisation of Baer factorisations via the indices of the centralisers of the Sylow subgroups of the factors.

Theorem 2.1.8. ([ $[\mathrm{B}$, Theorem $\mathrm{F}])$ Let $G=A B$ be the product of the subgroups $A$ and $B$. Then this is a Baer factorisation if and only if $\left|G: \mathbf{C}_{G}\left(A_{p}\right)\right|$ and $\left|G: \mathbf{C}_{G}\left(B_{p}\right)\right|$ are prime powers, for $A_{p} \in \operatorname{Syl}_{p}(A)$ and $B_{p} \in \operatorname{Syl}_{p}(B)$, and for every prime $p$.

We highlight the absence of any permutability condition on the factors in all the results presented in $[\vec{B}]$. Our contributions in this section are surveyed in $[\mathbf{2 6}]$, published in the Proceedings of Groups St Andrews 2017 in Birmingham.

\section{2 $\quad$ Zeros of irreducible characters and $\pi$-elements}

I

$\mathrm{n}$ the previous section we have frequently imposed arithmetical conditions on the class sizes of not all the elements in the factors of a factorised group, but only the ( $p$-regular) prime power order ones. Indeed, providing structural criteria for a group from certain subsets of its class sizes is a classical research area. In this spirit, this section is devoted to present the main results of $[\mathrm{C}]$ and $[\bar{D}]$, where we handle other families of elements in the factors of a factorised group. Concretely, vanishing elements and $\pi$-elements are involved, respectively. In both situations, core-factorisations of products of two groups have been revealed a fundamental tool.

Recently, numerous researchers have filtered the full set of class sizes of a group through its character table, and zeros of the irreducible characters have played an important role since the manuscript [29] of Isaac, Navarro and Wolf in 1999. In that paper, the authors introduced the next concept: an element $g$ of a group $G$ is said to be vanishing in $G$ whenever $\chi(g)=$ 0 for some irreducible character $\chi$ of $G$; otherwise $g$ is non-vanishing in $G$. These authors obtained elegant results that localise the non-vanishing elements of certain groups. Surprisingly, non-vanishing elements somehow violate the standard duality which frequently arises between 
the irreducible characters and the conjugacy classes of a group, since a non-linear irreducible complex character always vanishes on some element (this is a celebrated result in character theory due to Burnside, see [28. Theorem 3.15]). As an immediate consequence, it holds that a group has no vanishing elements if and only if it is abelian. Several problems concerning such elements have been addressed in the last few years. As a sample, groups with no vanishing $p$-elements were investigated by Dolfi, Pacifici, Sanus and Spiga in [23]; Moretó and Sangroniz classified in [32] groups whose irreducible characters vanish on "few" conjugacy classes. We refer the interested reader to the expository paper [22] for more information on the subject. It is to be said that the CFSG is usually needed in this development.

The terminus a quo of a current research on the so-called vanishing conjugacy class sizes (i.e. the class sizes of vanishing elements) could be situated in 2010, when Dolfi, Pacifici and Sanus stated that if a prime $p$ does not divide each vanishing class size of a group $G$, then $G$ has a normal $p$-complement and abelian Sylow $p$-subgroups ([21, Theorem A]). Notice that this is a "vanishing version" of the result of Chillag and Herzog mentioned in the Introduction: a group $G$ has a Sylow $p$-subgroup in its centre if and only if $p$ does not divide any class length of $G$. Later on, Brough and Kong addressed in [13, 14] some problems regarding square-free class sizes of vanishing elements (of prime power order), motivated by the results in [[18] mentioned in the previous section. Moreover, a graph associated to the primes that divide the vanishing class sizes is studied in [12] by Bianchi, Brough, Camina and Pacifici. Nevertheless, aside from those ones, no other arithmetical condition on such class sizes has been considered so far, which will probably lead to fruitful investigations in the coming years.

Our purpose in $[\overline{\mathrm{C}}]$ is to analyse the vanishing versions of some results concerning prime power class lengths, as well as to address the main results of [21] and [14] in the context of factorised groups. In this respect, we combine as a novelty the research on irreducible characters with the study of products of groups, since we obtain information about a factorised group from the vanishing columns of its character table that correspond to the elements in the factors. It is worthwhile noting that the product of two vanishing elements does not need to be vanishing in general. Moreover, an element in a (normal) subgroup can be vanishing in the whole group but not in that subgroup. Despite of these facts, non-vanishing elements behave well with respect to quotients groups, and non-abelian minimal normal subgroups always contain vanishing elements of the whole group. This suggests the significance of having at least one minimal normal subgroup contained in one of the factors for each quotient group, which is ensured for instance when the factors are (totally) mutually permutable (cf. [8. Theorem 4.3.11]). Since we also aim to weaken this permutability condition, we introduce in $[\bar{C}]$ the following concept.

Definition 2.2.1. Let $1 \neq G=A B$ be the product of the subgroups $A$ and $B$. We say that $G=$ $A B$ is a core-factorisation if for every proper normal subgroup $K$ of $G$ it holds that there exists a normal subgroup $1 \neq M / K$ of $G / K$ such that either $M / K \leqslant A K / K$ or $M / K \leqslant B K / K$.

This definition generalises products of two subgroups which are mutually permutable, as well as totally completely conditionally permutable (see Definition 1.2 and Lemma 2.5 of [4]). If we adopt the bar convention for the quotients over $K$, then the above condition means that core $_{\bar{G}}(\bar{A}) \operatorname{core}_{\bar{G}}(\bar{B}) \neq 1$, where $\operatorname{core}_{X}(H)$ denotes the core in a group $X$ of a subgroup $H$, i.e. the largest normal subgroup of $X$ contained in $H$. This illustrates the given name for such factorisations. It is not difficult to prove that factorisations of this kind are inherited by quotient groups. Further, we characterise in [C, Lemma 2] a core-factorisation of a factorised group $G=A B$ by means of the existence of a chief series of $G$ where all the chief factors are covered by either $A$ or $B$ (we recall that a subgroup $U$ covers a section $V / W$ of a group $G$ if $W(U \cap V)=V)$.

In $[\mathrm{D}]$ we show that this notion can also be approached somehow as a generalisation of the $\pi$-separability of a group. Observe that if $\pi$ is a set of primes and $G$ is a group that possesses 
both Hall $\pi$-subgroups and Hall $\pi^{\prime}$-subgroups, say $H$ and $L$ respectively, then $G=H L$ is a $\pi$-separable group if and only if for a chief series of $G$ it holds that each chief factor is covered by either $H$ or $L$. Therefore, we say that a product $G=A B$ is a core-factorisation whenever $G$ possesses a chief series such that each chief factor of $G$ is covered by either $A$ or $B$. We point out that this definition is equivalent to the one given before in virtue of [C, Lemma 2]. Moreover, it allows us to prove in [D] that if $G=A B$ is a core-factorisation of a $\pi$-separable group $G$, and $H=(H \cap A)(H \cap B)$ is a prefactorised Hall $\pi$-subgroup of $G$ with $H \cap A \in \operatorname{Hall}_{\pi}(A)$ and $H \cap B \in \operatorname{Hall}_{\pi}(B)$ (which always exists), then $H=(H \cap A)(H \cap B)$ is actually a core-factorisation.

Coming back to vanishing class sizes, we impose first prime power class length conditions on the $p$-elements in the factors of a core-factorisation that vanish in the whole group. In Theorem 2 of $[\overline{\mathrm{C}}]$ we prove that such groups are $p$-soluble of $p$-length at most 1 , and if all the considered class sizes are precisely $p$-powers for the same prime $p$, then the Sylow $p$-subgroup is unique. We also study this class length assumption for every prime power order element in the factors that vanishes in the whole group, obtaining the next theorem.

Theorem 2.2.2. ([C, Corollary 4]) Let $G=A B$ be a core-factorisation. If every prime power order element $x \in A \cup B$ vanishing in $G$ has prime power class size, then $G / \mathbf{F}(G)$ is abelian.

In particular, if these prime powers are actually p-numbers for a prime $p$, then $G$ has a normal Sylow p-subgroup and abelian Hall p'-subgroups.

In this case, when we take a trivial factorisation, we obtain new contributions for arbitrary groups not necessarily factorised. In addition, observe that these results form the vanishing versions of those in the previous section for a $(p$-)Baer factorisation. However, we emphasise that the techniques used in both approaches are totally different.

Our next contributions analyse to what extent the results in [21] and [14] remain true for a group with a core-factorisation. We first treat in [C, Theorem 3] core-factorisations $G=$ $A B$ such that every ( $p$-regular) prime power order element $x \in A \cup B$ vanishing in $G$ has conjugacy class not divisible by a fixed prime $p$. As an immediate consequence, we obtain a generalisation for prime power order elements of the main result of [21], although we use some similar techniques.

Regarding square-free class sizes of vanishing elements, we answer a posed question of Brough in [13] through the next vanishing version of Theorem 2.1.2.

Theorem 2.2.3. ([C, Theorem 4]) Let $G=A B$ be a core-factorisation, and let $p$ be a prime such that $(p-1,|G|)=1$. Suppose that $\left|x^{G}\right|$ is not divisible by $p^{2}$ for every prime power order element $x \in A \cup B$ vanishing in $G$. It follows that:

(1) $G$ is soluble.

(2) $G$ is p-nilpotent.

(3) If $P \in \operatorname{Syl}_{p}(G)$, then $P^{\prime} \leqslant \mathbf{\Phi}(P) \leqslant \mathbf{Z}(P)$, $P^{\prime}$ is elementary abelian and $\left|P^{\prime}\right| \leq p^{2}$.

The third assertion follows from the fact that in Theorem 2.1.3 it is enough to consider only the vanishing elements in the factors, since the non-vanishing ones are central due to [29. Theorem B].

It is not always possible to get analogous theorems to those about ordinary class sizes in the context of vanishing elements, and we show in $[\bar{C}]$ some examples in this line. An instance of this fact is provided by the next example about the corresponding assertion to Theorem 2.1.2 (3) in the vanishing case.

Example 2.2.4. [C, Example 5] Let $G=[A] B$ be the semidirect product of a cyclic group $B$ of order 4 which acts transitively on a cyclic group $A$ of order 5 . Let the prime $p=2$. Then 
$G=A B$ is a core-factorisation, and all the vanishing elements of $G$ (not only those lying in $A \cup B$ ) have class sizes not divisibly by 4 . However, $\mathbf{O}_{2}(G)=1$ and $G / \mathbf{O}_{2}(G)$ does not have elementary abelian Sylow 2-subgroups.

Brough and Kong also gave a supersolubility criterion for a group such that the class size of any vanishing prime power order element is square free ([14, Theorem 3.2]). Using different arguments, we not only get an extension of this fact for a group $G$ with a core-factorisation, but also enhance their result by giving more information on the structure of such a group: $G^{\prime}$ is abelian with elementary abelian Sylow subgroups, and $\mathbf{F}(G)^{\prime}$ has Sylow $p$-subgroups of order at most $p^{2}$ for each prime $p$ ([C, Theorem 7]). In fact, this result partially generalises Theorem 2.1.1 for vanishing elements of prime power order.

Within this research, we frequently need to understand the structure of factorised groups such that the factors contain no vanishing prime power order elements, and the next theorem turns out to be a crucial result. The CFSG is used via [C, Proposition 2].

Theorem 2.2.5. ([C, Corollary 3]) Let $G=A B$ be a core-factorisation. The following statements are pairwise equivalent:

(1) Every element $x \in A \cup B$ is non-vanishing in $G$.

(2) Every prime power order element $x \in A \cup B$ is non-vanishing in $G$

(3) $G$ is abelian.

As it has been said before, from a Burnside's result it is elementary to show that a group is abelian if and only if it has no vanishing elements. If we take a trivial factorisation in the previous theorem, then it follows that this last characterisation remains true when only prime power order elements are involved.

Finally, we put focus on the class lengths of other family of elements in the factors of a factorised group: the (prime power order) $\pi$-elements. More concretely, we summarise the most significant results of [D], in which we study the $\pi$-structure of products of groups when the class sizes of some $\pi$-elements in the factors are either $\pi$-numbers or $\pi^{\prime}$-numbers, for a set of primes $\pi$.

In Theorem A of $[\bar{D}]$ we prove a characterisation of $\pi$-decomposability for groups with a core-factorisation: if $G=A B$ is a core-factorisation, and $G$ possesses a Hall $\pi$-subgroup, then $\left|x^{G}\right|$ is a $\pi$-number for every prime power order $\pi$-element $x \in A \cup B$ if and only if $G=$ $\mathbf{O}_{\pi}(G) \times \mathbf{O}_{\pi^{\prime}}(G)$. We point out that the proof makes use of the knowledge on the automorphism groups of the non-abelian simple groups. An analogous result also appears in [35] for factorised groups $G=A B$ with one subnormal factor. We remark that, due to divisibility relations, if $A$ is the subnormal factor, then $A$ clearly inherits the class size assumptions. Further, there exists a normal subgroup of $G$ which contains $A$, so this normal subgroup is prefactorised. Note that groups with a core-factorisation and factorised groups with one subnormal factor are generally different types of groups.

The dual condition on the conjugacy class sizes of prime power order $\pi$-elements is also studied in [D, Proposition 1], i.e. when they are not divisible by any prime in $\pi$.

The reader may observe that under the assumptions of the mentioned [D, Theorem A], if a $\pi$-element in $A \cup B$ lies in the centre of a Hall $\pi$-subgroup of $G$, then this element has necessarily to be central in $G$ since its class size in $G$ is a $\pi$-number. Thus, a more general approach is to analyse products of groups where the $\pi$-elements in the factors have class sizes equal to either a $\pi$-number or a $\pi^{\prime}$-number. Precisely, in Theorem $\mathrm{B}$ of $[\mathrm{D}]$ we address this problem for a $\pi$-separable group that possesses a core-factorisation, and as a direct corollary we get the next result. 
Theorem 2.2.6. ([D, Corollary $\mathrm{C}])$ Let $G$ be a $\pi$-separable group. Then the following statements are pairwise equivalent:

(1) Each $\pi$-element $x \in G$ has class size either a $\pi$-number or a $\pi^{\prime}$-number.

(2) Either $G$ is $\pi$-decomposable or it has abelian Hall $\pi$-subgroups and its $\pi$-length is at most 1.

(3) For every $\pi$-element $x \in G$, either all $\left|x^{G}\right|$ are $\pi$-numbers or they are all $\pi^{\prime}$-numbers.

A similar condition was investigated in $[20]$ by Dolfi, who characterised the structure of groups all of whose class sizes are either $\pi$-numbers or $\pi^{\prime}$-numbers. He called such a group a class- $\pi$-separable group. In this spirit, we introduce in $[\mathrm{D}]$ a factorised-group version of this concept: $G=A B$ is a class- $\pi$-separable factorisation whenever $\left|x^{G}\right|$ is either a $\pi$-number or a $\pi^{\prime}$-number for every element $x \in A \cup B$. Observe that, a priori, we cannot affirm that if $G=A B$ is class- $\pi$-separable factorisation, then $A$ and $B$ are class- $\pi$-separable groups, since the sets $\pi\left(\left|x^{X}\right|\right)$ and $\pi\left(\left|x^{G}\right|\right)$ are not related in general, for some $x \in X \in\{A, B\}$. Surprisingly, if we further assume that $G=A B$ is a core-factorisation, then we determine that this phenomenon actually happens.

Theorem 2.2.7. ([D, Theorem $\mathrm{D}])$ Let $G=A B$ be the product of the subgroups $A$ and $B$, and assume that $G=A B$ is both a core-factorisation and a class- $\pi$-separable factorisation. Then, up to abelian direct factors, one of the following two possibilities holds for any $X \in\{A, B\}$ :

(1) $X$ is either a $\pi$-group or a $\pi^{\prime}$-group.

(2) Up to interchanging $\pi$ and $\pi^{\prime}, X=X_{\pi} X_{\pi^{\prime}}$ where $X_{\pi} \in \operatorname{Hall}_{\pi}(X), X_{\pi^{\prime}} \in \operatorname{Hall}_{\pi^{\prime}}(X)$, $X_{\pi^{\prime}} \unlhd X$, both $X_{\pi}$ and $X_{\pi^{\prime}}$ are abelian, and $X / \mathrm{O}_{\pi}(X)$ is a Frobenius group. Indeed, $\mathbf{O}_{\pi}(X)=\mathbf{Z}(X)$, the class sizes of $X$ are $\left\{1,\left|X_{\pi} / \mathbf{O}_{\pi}(X)\right|,\left|X_{\pi^{\prime}}\right|\right\}$, and $X$ is soluble.

In particular, both $A$ and $B$ are class- $\pi$-separable groups.

The mentioned characterisation of Dolfi now directly follows from the above theorem. We also present in $[\mathrm{D}]$ an example which shows that a core-factorisation of two class- $\pi$-separable groups may not be a class- $\pi$-separable factorisation.

To conclude, we also study in Theorem $\mathrm{E}$ of [D] groups with a core-factorisation such that all the $\pi$-elements in the factors have prime power class lengths, generalising the main result of [9]. Indeed, we provide an alternative proof of it, since the techniques used by those authors are based on a graph associated to the class sizes of the $\pi$-elements of a group. 


\title{
Concluding remarks and future investigations
}

\author{
Algebra and money are essentially levellers, the \\ first intellectually, the second effectively.
}

- Simone Weil

$S^{c}$ everal conclusions arise from the research developed within this dissertation. The main goal has been to study structural features of factorised groups via the conjugacy class sizes of some elements in the factors. The results presented are new contributions in this respect and, in many cases, they substantially improve already known ones. It is worth noting that these results produce a new way of filtering the whole set of class lengths of a group with the objective of obtaining structural properties of it. Moreover, further evidence is provided to confirm that the whole group structure is affected by the class sizes of ( $p$-regular) prime power order elements, as well as $\pi$-elements and vanishing elements. Through the consideration of this last kind of elements, it is combined as a novelty the research on factorised groups with representation and character theory, answering in particular an open question in [13]. Indeed, the approach considered in this dissertation can be viewed as a way of obtaining information about factorised groups that can be read from their character tables, concretely from the columns (i.e. class sizes) that correspond to the elements in the factors. In particular, the columns of the character table sometimes determine structural properties of the subgroups appearing in the factorisation of the group, as Theorems 2.1.6 and 2.2.7 reveal. Finally, the notion of corefactorisations of a group, introduced for the first time in this thesis, has turned out very useful, and it might be interesting by itself in the research on factorised groups.

Within this recent research line which combines products of groups and conjugacy classes, it can still be raised many interesting problems, on some of which we are currently working. A first inquiry is whether it is possible to relax, or even to remove, the permutability conditions imposed on the factors in some cases in the previous development. As a sample, in [D, Theorem A] we extend the following result in [7] due to Ballester-Bolinches, Cossey and Li: a mutually permutable product has a Sylow $p$-subgroup in its centre if and only if all the class lengths of the elements in the factors are not divisible by $p$, where $p$ is a fixed prime. Note that in [D. Theorem A] we consider a set of primes $\pi$ instead of $p^{\prime}$, and we weaken the assumption of having mutual permutable products by the consideration of core-factorisations. A certainly ambitious objective is to prove an analogous result for an arbitrary factorisation of two subgroups. A positive evidence may be based on the proof of the following non-simplicity conjecture: if the product $G=A B$ verifies that a fixed prime $p$ does not divide any class size of the elements in $A \cup B$, then $G$ is non-simple. The use of the CFSG in this context seems unavoidable. We have 
obtained some partial positive results concerning this conjecture, for example when either $p=2$ or $p$ coincides with the characteristic of the field in a simple group of Lie type.

Besides, there are other questions which arise from our contributions that still remain open. For instance, regarding square-free class sizes, we recall that in [7] it is also proved that if a product $G=A B$ of two mutually permutable subgroups satisfies that $p^{2}$ does not divide each class size of a $p$-regular element in $A \cup B$, then a Sylow $p$-subgroup of each chief factor of $G$ has order at most $p$. In particular, if $G$ is $p$-soluble, then $G$ is $p$-supersoluble. In Theorem C of [A] we generalise the second assertion through the consideration of only $p$-regular prime power order elements in the factors. However, in this situation we have not been able to demonstrate the first claim. The core of the proof of Ballester-Bolinches, Cossey and $\mathrm{Li}$ is the next result in [16], which relies heavily on the CFSG: if a fixed prime $p$ divides $\left|\mathbf{C}_{G}(x)\right|$ for all $x \in G$, then $G$ is not a non-abelian simple group. An open question of interest by itself is whether this last result is valid when only prime power order elements are involved. If $p$ is the largest prime divisor of the order of $G$, then so is certainly in virtue of [1], Theorem 1.3].

Concerning prime power class lengths, in Theorem 2.1.6 we showed that in a Baer factorisation $G=A B$ there exists a divisibility relation between the class sizes of the prime power order elements in the factors and the corresponding class sizes in the whole group. As a consequence, $A$ and $B$ are Baer groups. We have provided in Example 2.1.7 a 2-Baer factorisation $G=A B$ where $\left|x^{A}\right|=3$ and $\left|x^{G}\right|=7$ for a 2-element $x \in A$, so clearly the conclusion of Theorem 2.1.6 fails in this case. However, the class size in the factor $A$ is still a prime power, which leads to wonder whether the factors of a $p$-Baer factorisation are also $p$-Baer groups.

If we focus on the zeros of the irreducible characters, then it has not been yet tackled the vanishing version of Theorem 2.1.5 about the uniqueness of the primes that divide the class lengths, even for an arbitrary group not necessarily factorised. More concretely, when all the vanishing $p$-elements of a group have prime power class sizes, is it true that there exists a unique prime $q$ such that all the class sizes are $q$-powers?

In view of the developments presented in the previous sections, it is also natural to consider the class sizes of other families of elements in the factors. For instance, it may be interesting to analyse the influence on the whole group structure of the class sizes of real elements of a factorised group that lie in the factors. We recall that an element $x$ of a group $G$ is said to be a real element of $G$ if it is conjugate to its inverse. Equivalently, $x$ is a real element of $G$ if and only if every irreducible character of $G$ takes a real value on $x$, so again we are filtering the full set of class sizes of $G$ by its character table. The properties of this type of elements, and in particular of their class sizes, have been extensively examined in the literature. Notice that in the factorised-group case, a real element of the whole group that lie in a factor might not be a real element of that factor.

The imposition of other arithmetical properties on the class lengths gives rise to new attractive problems. In this sense, Cossey and $\mathrm{Li}$ investigated in [17] (which cites our work [A]) products of two mutually permutable subgroups whose prime power order elements in the factors have class sizes not divisible by $p^{p-1}$, for a given prime $p$. We are currently analysing whether the mutual permutability hypothesis can be weakened by considering a core-factorisation. Another arithmetical condition that may be intriguing is when the class sizes of the elements in the factors have the same $p$-part, i.e. when the greatest $p$-power that divides each of these class lengths is unique.

With respect to core-factorisations $G=A B$, we can construct the following normal series which may be useful for further investigations: $1 \unlhd \mathfrak{C}_{A}(G) \unlhd \mathfrak{C}_{A, B}(G) \unlhd \mathfrak{C}_{A, B, A}(G) \unlhd \cdots \unlhd G$, where $\mathfrak{C}_{A}(G):=\operatorname{core}_{G}(A), \mathfrak{C}_{A, B}(G) / \mathfrak{C}_{A}(G):=\operatorname{core}_{G / \mathfrak{C}_{A}(G)}\left(B \mathfrak{C}_{A}(G) / \mathfrak{C}_{A}(G)\right.$ ), etc. This series reachs $G$ due to [D, Lemmas 1 and 2]. The length of this series and its bounds possibly control the global structure of $G$. 
Finally, a general issue is to extend the development exposed so far to products of more than two groups. As a sample, the aforementioned result in [7] on the $p$-decomposability of a mutually permutable product is actually proved for $n$ factors. Probably, the concept of corefactorisations for $n \geq 2$ factors may play an important role, although prefactorised Sylow subgroups might not automatically exist in this scene (as mentioned in the Introduction). 



\section{Bibliography}

[1] B. Amberg, A. Carocca, and L.S. Kazarin. "Criteria for the solubility and non-simplicity of finite groups". $J$. Algebra 285 (2005) 58-72.

[2] B. Amberg, S. Franciosi, and F. de Giovanni. Products of groups. Oxford University Press Inc., 1992.

[3] M. Arroyo-Jordá, P. Arroyo-Jordá, A. Martínez-Pastor, and M.D. Pérez-Ramos. "On conditional permutability and factorized groups". Ann. Mat. Pura Appl. 193 (2014) 1123-1138.

[4] M. Arroyo-Jordá, P. Arroyo-Jordá, and M.D. Pérez-Ramos. "On conditional permutability and saturated formations". Proc. Edinb. Math. Soc. 54 (2011) 309-319.

[5] M. Asaad and A. Shaalan. "On the supersolvability of finite groups". Arch. Math. (Basel) 53 (1989) 318-326.

[6] R. Baer. "Group elements of prime power index". Trans. Amer. Math. Soc. 75 (1953) 20-47.

[7] A. Ballester-Bolinches, J. Cossey, and Y. Li. "Mutually permutable products and conjugacy classes". Monatsh. Math. 170 (2013) 305-310.

[8] A. Ballester-Bolinches, R. Esteban-Romero, and M. Asaad. Products of finite groups. Vol. 53. W. de Gruyter Expositions In Mathematics, 2010.

[9] A. Beltrán and M. J. Felipe. "Prime powers as conjugacy class lengths of $\pi$-elements". Bull. Austral. Math. Soc. 69 (2004) 317-325.

[10] A. Beltrán, M. J. Felipe, G. Malle, A. Moretó, G. Navarro, L. Sanus, R. Solomon, and P. H. Tiep. "Nilpotent and abelian Hall subgroups in finite groups". Trans. Amer. Math. Soc. 368 (2016) 2497-2513.

[11] Y. Berkovich and L. S. Kazarin. "Indices of elements and normal structure of finite groups". J. Algebra 283 (2005) 564-583.

[12] M. Bianchi, J. Brough, R.D. Camina, and E. Pacifici. "On vanishing class sizes in finite groups". J. Algebra 489 (2017) 446-459.

[13] J. Brough. "On vanishing criteria that control finite group structure". J. Algebra 458 (2016) 207-215.

[14] J. Brough and Q. Kong. "On vanishing criteria that control finite group structure II". Bull. Aust. Math. Soc. 98 (2018) 251-257.

[15] A. R. Camina and R. D. Camina. "Implications of conjugacy class size". J. Group Theory 1 (1998) 257-269.

[16] D. Chillag and M. Herzog. "On the length of the conjugacy classes of finite groups". J. Algebra 131 (1990) $110-125$.

[17] J. Cossey and Y. Li. "On the structure of a mutually permutable product of finite groups". Acta Math. Hungar. 154 (2018) 525-529.

[18] J. Cossey and Y. Wang. "Remarks on the length of conjugacy classes of finite groups". Comm. Algebra 27 (1999) 4347-4353.

[19] K. Doerk and T. Hawkes. Finite Soluble Groups. Vol. 4. W. de Gruyter Expositions In Mathematics, 1992.

[20] S. Dolfi. "Arithmetical conditions on the length of the conjugacy classes of a finite group". J. Algebra 174 (1995) 753-771.

[21] S. Dolfi, E. Pacifici, and L. Sanus. "Groups whose vanishing class sizes are not divisible by a given prime". Arch. Math. (Basel) 94 (2010) 311-317.

[22] S. Dolfi, E. Pacifici, and L. Sanus. "On Zeros of Characters of Finite Groups”. Ed. by Sastry N. and Yadav M. Group Theory and Computation, Indian Statistical Institute Series. Springer Nature Singapore, (2018) 41-58.

[23] S. Dolfi, E. Pacifici, L. Sanus, and P. Spiga. "On the orders of zeros of irreducible characters". J. Algebra 321 (2009) 345-352. 
[24] B. Fein, W. M. Kantor, and M. Schacher. "Relative Brauer groups II". J. Reine Angew. Math. 328 (1981) 39-57.

[25] M. J. Felipe, A. Martínez-Pastor, and V. M. Ortiz-Sotomayor. "On finite groups with square-free conjugacy class sizes". Int. J. Group Theory 7 (2018) 17-24.

[26] M. J. Felipe, A. Martínez-Pastor, and V. M. Ortiz-Sotomayor. "Structural criteria in factorised groups via conjugacy class sizes". Ed. by C. M. Campbell et al. Vol. 455. Proceedings of Groups St. Andrews 2017 in Birmingham, London Math. Soc. Lecture Note Series. Cambridge University Press, (2019) 289-299.

[27] W. Guo, K.P. Shum, and A.N. Skiba. "Conditionally permutable subgroups and supersolubility of finite groups". South. Asian Bull. Math. 29 (2005) 493-510.

[28] I. M. Isaacs. Character theory of finite groups. Academic Press Inc., 1976.

[29] I. M. Isaacs, G. Navarro, and T. R. Wolf. "Finite group elements where no irreducible character vanishes". $J$. Algebra 222 (1999) 413-423.

[30] H. G. Knoche. "Über den Frobeniusschen Klassenbegriff in nilpotenten Gruppen”. Math. Z. 55 (1951) 7183.

[31] X. Liu, Y. Wang, and H. Wei. "Notes on the length of conjugacy classes of finite groups". J. Pure Appl. Algebra 196 (2005) 111-117.

[32] A. Moretó and J. Sangroniz. "On the number of conjugacy classes of zeros of characters". Israel J. Math. 142 (2004) 163-187.

[33] G. Qian and Y. Wang. "On conjugacy class sizes and character degrees of finite groups”. J. Algebra Appl. 13 (2014) 1350100 (9 pages).

[34] The GAP Group, GAP - Groups, Algorithms, and Programming, Version 4.10.0. 2018. URL: https:// Www.gap-system.org.

[35] X. H. Zhao, X. Y. Guo, and J. Y. Shi. "On the conjugacy class sizes of prime power order $\pi$-elements". Southeast Asian Bull. Math. 35 (2011) 735-740. 
It is tribute to the genius of Galois that he recognised that those subgroups for which the left and right cosets coincide are distinguished ones. Very often in mathematics the crucial problem is to recognise and to discover what are the relevant concepts; once this is accomplished the job may be more than half done.

— ISRAEl Nathan Herstein 



\title{
Square-free class sizes in products of groups
}

\author{
M. J. FELIPE · A. MARTÍNEZ-PASTOR · V. M. ORTIZ-SOTOMAYOR*
}

\begin{abstract}
We obtain some structural properties of a factorised group $G=A B$, given that the conjugacy class sizes of certain elements in $A \cup B$ are not divisible by $p^{2}$, for some prime $p$. The case when $G=A B$ is a mutually permutable product is specially considered.
\end{abstract}

Keywords Finite groups $\cdot$ Soluble groups $\cdot$ Products of subgroups $\cdot$ Conjugacy classes

2010 MSC $20 \mathrm{D} 10 \cdot 20 \mathrm{D} 40 \cdot 20 \mathrm{E} 45$

\section{Introduction}

All groups considered throughout this paper are finite. Over the last years, many authors have investigated the influence of conjugacy class sizes on the structure of finite groups. In the meantime, numerous studies in the framework of group theory have focused in factorised groups. In this setting, a central question is how the structure of the factors affects the structure of the whole group, in particular when they are connected by certain permutability properties. The purpose of this paper is to show new achievements which combine both current perspectives in finite groups. More precisely, our aim is to get some information about a factorised group, provided that the conjugacy class sizes of some elements of its factors are square-free.

The earlier starting point of our investigation can be traced back to the paper of Chillag and Herzog $([\overline{5}])$, where the structure of a group in which all elements have square-free conjugacy class sizes was first analysed. Next, in [6], Cossey and Wang localised one of the main theorems in [5] for a fixed prime $p$, that is, they considered conjugacy class sizes not divisible by $p^{2}$, for certain prime $p$. Later on, this study was improved by Li in [11], and by Liu, Wang, and Wei in [12], by replacing conditions on all conjugacy classes by those referring only to conjugacy classes of either $p$-regular elements or prime power order elements, using the classification theorem of finite simple groups (CFSG). These authors also first obtained some preliminary results

\footnotetext{
*The first author is supported by Proyecto Prometeo II/2015/011, Generalitat Valenciana (Spain), and the second author is supported by Proyecto MTM2014-54707-C3-1-P, Ministerio de Economía, Industria y Competitividad (Spain). The results in this paper are part of the third author's Ph.D. thesis, and he acknowledges the predoctoral grant ACIF/2016/170, Generalitat Valenciana (Spain). The authors wish to thank John Cossey for helpful conversations during his last visit to Valencia.
}

Instituto Universitario de Matemática Pura y Aplicada (IUMPA-UPV), Universitat Politècnica de València, Camino de Vera s/n, 46022, Valencia, Spain.

凶: mfelipe@mat.upv.es, anamarti@mat.upv.es, vicorso@doctor.upv.es

ORCID iDs: 0000-0002-6699-3135, 0000-0002-0208-4098, 0000-0001-8649-5742 
in factorised groups. This research was extended in 2012 by Ballester-Bolinches, Cossey and $\mathrm{Li}$ in [2], through mutually permutable products. More recently, in 2014, Qian and Wang ([13]) have gone a step further by considering just conjugacy class sizes of $p$-regular elements of prime power order (although not in factorised groups).

In the context of factorised groups, and aiming to obtain criteria for products of supersoluble subgroups to be supersoluble, several authors have considered products in which certain subgroups of the factors permute (see $[\mathbf{3}]$ for a detailed account). In this scene, we are interested in mutually permutable products, factorised groups $G=A B$ such that the subgroups $A$ and $B$ are mutually permutable, i.e., $A$ permutes with every subgroup of $B$ and $B$ permutes with every subgroup of $A$ (see also [4]). Obviously, if $A$ and $B$ are normal in $G$, then they are mutually permutable.

We recall that, for a group $G$, the set $x^{G}=\left\{g^{-1} x g: g \in G\right\}$ is the conjugacy class of the element $x \in G$, and $\left|x^{G}\right|$ denotes the conjugacy class size of $x$. If $p$ is a prime number, we say that $x \in G$ is a $p$-regular element if its order is not divisible by $p$, and that it is a $p$-element if its order is a power of $p$. Moreover, if $n$ is an integer, let $n_{p}$ denote the highest power of $p$ dividing $n$. The $m$ th group of order $n$ in the SmallGroups library [14] of GAP will be identified by $n \# m$. The remaining notation is standard and is taken mainly from [7]. We also refer to this book for details about classes of groups.

In this paper, motivated by the above development, at first we focus on the case of $p$-groups, extending for factorised groups the well-known Knoche's theorem (see [10]).

Theorem A. Let $p$ be a prime number and let $P=A B$ be a p-group such that $p^{2}$ does not divide $\left|x^{P}\right|$ for all $x \in A \cup B$. Then $P^{\prime} \leqslant \mathbf{\Phi}(P) \leqslant \mathbf{Z}(P), P^{\prime}$ is elementary abelian and $\left|P^{\prime}\right| \leq p^{2}$.

Our next goal in the paper is to prove the following theorem, regarding mutually permutable products.

Theorem B. Let $G=A B$ be the mutually permutable product of the subgroups $A$ and $B$, and let $p$ be a prime such that $\operatorname{gcd}(p-1,|G|)=1$. If $p^{2}$ does not divide $\left|x^{G}\right|$ for any $p$-regular element $x \in A \cup B$ of prime power order, then:

(1) $G$ is soluble.

(2) $G$ is p-nilpotent.

(3) The Sylow p-subgroups of $G / \mathbf{O}_{p}(G)$ are elementary abelian.

In the particular case when $G=A=B$, we recover [13, Theorem A] (see Section 3 , Corollary 11. We remark that both results use the CFSG.

Moreover, in relation to the third assertion, we have found a gap in one of the statements in [6. Theorem 1], as it is reported in Remark11(a).

On the other hand, we point out that it is possible to find examples of groups factorised as a product of two (mutually permutable) subgroups which satisfy the hypotheses of Theorem $B$ for some fixed prime $p$ and, however, there exist elements $x \in A \cup B$ such that $p^{2}$ divides either $\left|x^{A}\right|$ or $\left|x^{B}\right|$ (see Remark 1 (b)).

The next theorem generalises the last assertion of [2, Theorem 1.3] regarding $p$-soluble groups, by considering only prime power order elements:

Theorem C. Let $G=A B$ be the mutually permutable product of the subgroups $A$ and $B$, and let $p$ be a prime. Suppose that for every prime power order p-regular element $x \in A \cup B,\left|x^{G}\right|$ is not divisible by $p^{2}$. If $G$ is $p$-soluble, then $G$ is p-supersoluble.

In the line of [5, Theorem 1] and [6, Theorem 2], if we consider all prime numbers, then we obtain some information about the structure of the derived subgroup of a factorised group $G$. 
Theorem D. Let $G=A B$ be the product of the subgroups $A$ and $B$, and assume that $G$ is supersoluble. Suppose that every prime power order element $x \in A \cup B$ has square-free conjugacy class size. Then:

(1) $G^{\prime}$ is abelian.

(2) The Sylow subgroups of $G^{\prime}$ are elementary abelian.

(3) $\mathbf{F}(G)^{\prime}$ has Sylow p-subgroups of order at most $p^{2}$, for every prime $p$.

If we limit our conditions only to $p$-regular elements, as a consequence of Theorems $B$ and C. we obtain the following result which extends [2, Corollary 1.5] (see Corollary 3) for prime power order elements, and also a theorem of [11], for products of groups.

Theorem E. Let $G=A B$ be the mutually permutable product of the subgroups $A$ and $B$. Suppose that for every prime $p$ and every prime power order p-regular element $x \in A \cup B$, $\left|x^{G}\right|$ is not divisible by $p^{2}$. Then $G$ is supersoluble, and $G / \mathbf{F}(G)$ has elementary abelian Sylow subgroups.

We remark that the first statement in Theorem $\mathrm{D}$ is not further true under the weaker hypotheses of the above theorem, even for arbitrary groups not necessarily factorised, as pointed out in [13]. Indeed, as a result of Theorem $\mathrm{E}$, the supersolubility condition in Theorem $\mathrm{D}$ can be exchanged by the mutual permutability of the factors.

On the other hand, with the stronger assumption that all $p$-regular elements of the factors (not only those of prime power order) have conjugacy class sizes not divisible by $p^{2}$, we get extra information about the orders of the Sylow $p$-subgroups of $G / \mathbf{F}(G)$, extending partially [ $\mathbf{6}$. Theorem 2].

Theorem F. Let $G=A B$ be the mutually permutable product of the subgroups $A$ and $B$. Suppose that for every prime $p$ and every p-regular element $x \in A \cup B,\left|x^{G}\right|$ is not divisible by $p^{2}$. Then the order of a Sylow p-subgroup of $G / \mathbf{F}(G)$ is at most $p^{2}$.

In summary, when dealing with mutually permutable products, the next corollary follows directly from the above theorems.

Corollary G. Let $G=A B$ be the mutually permutable product of the subgroups $A$ and $B$. Suppose that $\left|x^{G}\right|$ is square-free for each element $x \in A \cup B$. Then $G$ is supersoluble, and both $G / \mathbf{F}(G)$ and $G^{\prime}$ have elementary abelian Sylow subgroups. Moreover, $G^{\prime}$ is abelian, and both groups $G / \mathbf{F}(G)$ and $\mathbf{F}(G)^{\prime}$ have Sylow p-subgroups of order at most $p^{2}$, for each prime $p$.

In Section 3 we prove Theorems $\mathrm{A}$ B and $\mathrm{C}$, which refer to class sizes not divisible by $p^{2}$, for a fixed prime $p$. Theorems $\mathrm{D}, \mathrm{E}$ and $\mathrm{F}$, which consider square-free conjugacy class sizes (for all primes), are proved in Section 4 . In both cases we will illustrate the scope of the results presented with some examples.

\section{Preliminary results}

We use the following elementary properties frequently, sometimes without further reference.

Lemma 1. Let $N$ be a normal subgroup of a group $G$, and let $p$ be a prime. Then:

(a) $\left|x^{N}\right|$ divides $\left|x^{G}\right|$, for any $x \in N$.

(b) $\left|(x N)^{G / N}\right|$ divides $\left|x^{G}\right|$, for any $x \in G$.

(c) If $x N$ is a p-element of $G / N$, then there exists a p-element $x_{1} \in G$ such that $x N=x_{1} N$. 
We need specifically the following fact about Hall subgroups of factorised groups. It is a reformulation of [1, 1.3.2] which is convenient for our purposes. We recall that a group is a $\mathrm{D}_{\pi}$-group, for a set of primes $\pi$, if every $\pi$-subgroup is contained in a Hall $\pi$-subgroup, and any two Hall $\pi$-subgroups are conjugate. In particular, all $\pi$-separable groups are $\mathrm{D}_{\pi}$-groups for any set of primes $\pi$, and all groups are $\mathrm{D}_{\pi}$-groups when $\pi$ consists of a single prime.

Lemma 2. Let $G=A B$ be the product of the subgroups $A$ and $B$. Assume that $A, B$, and $G$ are $D_{\pi}$-groups for a set of primes $\pi$. Then there exists a Hall $\pi$-subgroup $H$ of $G$ such that $H=(H \cap A)(H \cap B)$, with $H \cap A$ a Hall $\pi$-subgroup of $A$ and $H \cap B$ a Hall $\pi$-subgroup of $B$.

We collect here some results on mutually permutable products, which will be very useful along the paper.

Lemma 3. Let the group $G=A B$ be the product of the mutually permutable subgroups $A$ and $B$. Then we have:

(a) $([3,4.1 .10]) G / N$ is the product of the mutually permutable subgroups $A N / N$ and $B N / N$.

(b) ([3, 4.1.21]) If $U$ is a subgroup of $G$, then $(U \cap A)(U \cap B)$ is a subgroup, and $U \cap A$ and $U \cap B$ are mutually permutable. Moreover, if $N$ is a normal subgroup of $G$, then $(N \cap A)(N \cap B)$ is also normal in $G$.

Theorem 1. ([4, Theorem 1]) Let the non-trivial group $G=A B$ be the product of the mutually permutable subgroups $A$ and $B$. Then $A_{G} B_{G}$ is not trivial.

The following lemma will be essential in the proofs of our theorems.

Lemma 4. ([2, Lemma 2.4]) Let $p$ be a prime, and $Q$ be a $p^{\prime}$-group acting faithfully on an elementary abelian $p$-group $N$ with $|[x, N]|=p$, for all $1 \neq x \in Q$. Then $Q$ is cyclic.

The next result is the first assertion of Theorem A in [13], which uses the CFSG.

Theorem 2. Let $G$ be a group. For a fixed prime $p$ with $\operatorname{gcd}(p-1,|G|)=1$, if $p^{2}$ does not divide $\left|x^{G}\right|$ for any p-regular element $x \in G$ of prime power order, then $G$ is soluble.

Finally, the later lemma, which is a nice result due to Isaacs, will be very useful in the proof of Theorem $\mathrm{D}$

Lemma 5. ([9, 4.17]) Let $K$ be an abelian normal subgroup of a finite group $G$, and let $x \in G$ be non-central. Then $\left|\mathbf{C}_{G}(x)\right|<\left|\mathbf{C}_{G}(y)\right|$, where $y=[k, x]$ and $k \in K$ is arbitrary.

\section{Class sizes not divisible by $p^{2}$, for a fixed prime $p$}

The well-known Knoche's theorem (see [10] ) asserts that if $P$ is a $p$-group, $p$ a prime, then the conjugacy class sizes of $P$ are square-free if, and only if, $\left|P^{\prime}\right| \leq p$. We begin this section by proving Theorem $\mathrm{A}$, which clearly extends it for factorised groups.

Proof of Theorem A. Since $\left|P: \mathbf{C}_{P}(x)\right| \leq p$ for each $x \in A \cup B$, it follows $\boldsymbol{\Phi}(P) \leqslant \mathbf{C}_{P}(x)$. Therefore, $\boldsymbol{\Phi}(P)$ commutes with both $A$ and $B$, so $P^{\prime} \leqslant \boldsymbol{\Phi}(P) \leqslant \mathbf{Z}(P)$. Hence $P / \mathbf{Z}(P)$ is elementary abelian, and $x^{p} \in \mathbf{Z}(P)$ for all $x \in P$. Since $P^{\prime} \leqslant \mathbf{Z}(P)$ and $[x, y]^{p}=\left[x^{p}, y\right]=1$ (see [7, A - 7.3(a)]) for any $x, y \in P$, it follows that $P^{\prime}$ is elementary abelian. Now it remains to prove that $\left|P^{\prime}\right| \leq p^{2}$.

Let $[x, y]$ be a generator of $P^{\prime}$. Since $P^{\prime} \leqslant \mathbf{Z}(P)$ and $y=y_{a} y_{b}$ with $y_{a} \in A$ and $y_{b} \in B$, then $[x, y]=\left[x, y_{a} y_{b}\right]=\left[x, y_{b}\right]\left[x, y_{a}\right]^{y_{b}}=\left[x, y_{b}\right]\left[x, y_{a}\right] \in[P, B][P, A]$. Thus $P^{\prime}=[P, B][P, A]$. 
Clearly, $[P, B]$ is elementary abelian. Suppose $[P, B] \neq 1$, and let $1 \neq[x, z]$ and $1 \neq\left[x^{\prime}, z^{\prime}\right]$ be two generators of $[P, B]$, with $x, x^{\prime} \in P$ and $z, z^{\prime} \in B$. We distinguish three cases in order to prove that $\langle[x, z]\rangle=\left\langle\left[x^{\prime}, z^{\prime}\right]\right\rangle$ :

i) Suppose first $z, z^{\prime} \in B \backslash \mathbf{Z}(B)$. Let $b \in B \backslash\left(\mathbf{C}_{P}(z) \cup \mathbf{C}_{P}\left(z^{\prime}\right)\right)$. Since $\left|P: \mathbf{C}_{P}(z)\right|=p$ then $P=\mathbf{C}_{P}(z)\langle x\rangle$. Moreover, $b \notin \mathbf{C}_{P}(z)$ implies that $1 \neq[b, z]=\left[x^{i} t, z\right]=\left[x^{i}, z\right]=[x, z]^{i}$, where $b=t x^{i}$ with $t \in \mathbf{C}_{P}(z)$. On the other hand, $P=\mathbf{C}_{P}(b)\langle z\rangle$ so $z^{\prime}=z^{j} k$, with $k \in \mathbf{C}_{P}(b)$. Hence $1 \neq\left[b, z^{\prime}\right]=\left[b, z^{j} k\right]=[b, z]^{j}=[x, z]^{i+j}$. Finally, as $b \in P=\mathbf{C}_{P}\left(z^{\prime}\right)\left\langle x^{\prime}\right\rangle$, then $b=\left(x^{\prime}\right)^{m} s$ with $s \in \mathbf{C}_{P}\left(z^{\prime}\right)$. Therefore $1 \neq[x, z]^{i+j}=\left[b, z^{\prime}\right]=\left[\left(x^{\prime}\right)^{m} s, z^{\prime}\right]=\left[x^{\prime}, z^{\prime}\right]^{m}$, and recall that $[x, z]$ and $\left[x^{\prime}, z^{\prime}\right]$ both have order $p$. Thus, $\langle[x, z]\rangle=\left\langle\left[x^{\prime}, z^{\prime}\right]\right\rangle$.

ii) Now suppose $z, z^{\prime} \in \mathbf{Z}(B)$. Then $B \leqslant \mathbf{C}_{P}(z) \cap \mathbf{C}_{P}\left(z^{\prime}\right)$. There exists $w \in P \backslash\left(\mathbf{C}_{P}(z) \cup\right.$ $\left.\mathbf{C}_{P}\left(z^{\prime}\right)\right)$. Therefore, $w=w_{a} w_{b}$ with $w_{a} \in A$ and $w_{b} \in B \leqslant \mathbf{C}_{P}(z) \cap \mathbf{C}_{P}\left(z^{\prime}\right)$, so $w_{a} \in$ $A \backslash\left(\mathbf{C}_{P}(z) \cup \mathbf{C}_{P}\left(z^{\prime}\right)\right)$. Arguing analogously as in case i) with $w_{a}$ instead of $b$, we conclude that $\langle[x, z]\rangle=\left\langle\left[x^{\prime}, z^{\prime}\right]\right\rangle$ too.

iii) Finally, suppose $z \in B \backslash \mathbf{Z}(B)$ but $z^{\prime} \in \mathbf{Z}(B)$. Let $z^{\prime \prime}=z z^{\prime} \in B \backslash \mathbf{Z}(B)$. Therefore, we have $\left[x^{\prime}, z^{\prime \prime}\right]=\left[x^{\prime}, z^{\prime}\right]\left[x^{\prime}, z\right]$. If $\left[x^{\prime}, z^{\prime \prime}\right]=1$ then $1 \neq\left[x^{\prime}, z^{\prime}\right]^{-1}=\left[x^{\prime}, z\right]$, and applying case i) to both $[x, z]$ and $\left[x^{\prime}, z\right]$ we conclude that they generate the same cyclic group of order $p$. On the other hand, if $\left[x^{\prime}, z^{\prime \prime}\right] \neq 1$ and $\left[x^{\prime}, z\right]=1$, then we apply again the first case. Finally, if both $\left[x^{\prime}, z^{\prime \prime}\right] \neq 1 \neq\left[x^{\prime}, z\right]$ then they generate the same cyclic group by case i) again. Thus $1 \neq\left\langle\left[x^{\prime}, z^{\prime}\right]\right\rangle=\left\langle\left[x^{\prime}, z^{\prime \prime}\right]\left[x^{\prime}, z\right]^{-1}\right\rangle \leqslant\left\langle\left[x^{\prime}, z^{\prime \prime}\right]\right\rangle$. Since the last one has order $p$, it follows $\left\langle\left[x^{\prime}, z^{\prime}\right]\right\rangle=\left\langle\left[x^{\prime}, z^{\prime \prime}\right]\right\rangle$. So we have $\left\langle\left[x^{\prime}, z^{\prime}\right]\right\rangle=\left\langle\left[x^{\prime}, z^{\prime \prime}\right]\right\rangle$, which is equal to $\langle[x, z]\rangle$ by i) again.

In conclusion, if $[P, B] \neq 1$, then it has order $p$. Analogously with $[P, A]$. Hence $\left|P^{\prime}\right|=$ $|[P, B][P, A]| \leq p^{2}$ and this establishes the result.

Example 1. The converse of the above result is not true in general, in contrast to Knoche's theorem. Let $P$ be the group of the Small groups library of GAP with identification number $32 \# 35$, which is the product of a cyclic group of order 4 and a quaternion group of order 8 . Then its derived group is $P^{\prime}=C_{2} \times C_{2}$, and $P^{\prime}=\boldsymbol{\Phi}(P)=\mathbf{Z}(P)$. Nevertheless, there are elements in the quaternion group with conjugacy class size equal to 4 .

Example 2. Let $G=Q_{8} \times D_{8}$ be the direct product of a quaternion group and a dihedral group of order 8 . Then every element contained in each factor has conjugacy class size equal to either 1 or 2, so Theorem Aapplies. However, there are elements in $G$ with conjugacy class size divisible by 4 , and Knoche's result cannot be applied.

Now we proceed with a key result in the sequel.

Proposition 1. Let $G$ be a group, and let $p$ be a prime. Suppose that $N$ is an abelian minimal normal subgroup of $G$, which is a $p^{\prime}$-group. Then:

(1) If $G$ is p-nilpotent, and $\left|x^{G}\right|$ is not divisible by $p^{2}$ for every element $x \in N$, then $\left|\mathbf{O}_{p}\left(G / N \mathbf{O}_{p}(G)\right)\right| \leq p$.

(2) If $K / N \mathbf{O}_{p}(G)=\mathbf{O}_{p}\left(G / N \mathbf{O}_{p}(G)\right)$ has order $p$, and $P$ is a Sylow $p$-subgroup of $K$, then $\mathbf{C}_{N}(P)=1$.

Proof. (1) Suppose that the result is not true, and let $G$ be a counterexample of minimal order. Since the hypotheses are inherited by quotients, we may assume by standard arguments that $\mathbf{O}_{p}(G)=1$, and then also $\boldsymbol{\Phi}(G)=1$. Since $N$ is abelian, by a Gaschütz's result ([8,4.4]) $N$ is complemented, that is, $G=N S$ with $N \cap S=1$. We may assume that $G / N$ is not a $p^{\prime}$-group, so $\mathbf{O}_{p}(G / N) \cong \mathbf{O}_{p}(S) \neq 1$ by the minimality of $G$. Let $P$ be a Sylow $p$-subgroup of $S$ (so $P$ is a Sylow $p$-subgroup of $G$ ). Hence it follows $\mathbf{O}_{p}(S) \cap \mathbf{Z}(P) \neq 1$. Let $Z$ be a minimal normal subgroup of $\mathbf{O}_{p}(S) \cap \mathbf{Z}(P)$. Since $S$ is $p$-nilpotent, we get $S=P L$, where $L$ is normal in $S$ and $P \cap L=1$. It follows that $[L, Z] \leqslant\left[L, \mathbf{O}_{p}(S)\right] \leqslant L \cap \mathbf{O}_{p}(S)=1$, so 
$Z \leqslant \mathbf{Z}(S)$. Note that $\mathbf{C}_{N}(Z)$ is normal in $G=S N$. Consequently, by the minimality of $N$, we have either $\mathbf{C}_{N}(Z)=1$ or $\mathbf{C}_{N}(Z)=N$. If $\mathbf{C}_{N}(Z)=N$, then $Z \leqslant \mathbf{Z}(G)$, which implies that $Z \leqslant \mathbf{O}_{p}(G)=1$, a contradiction. So we may affirm $\mathbf{C}_{N}(Z)=1$, for every minimal normal subgroup $Z$ of $\mathbf{O}_{p}(S) \cap \mathbf{Z}(P)$.

Now let $1 \neq x \in N$ such that a Sylow $p$-subgroup of $\mathbf{C}_{G}(x)$, say $P_{0}$, is contained in $P$, so $P_{0}=\mathbf{C}_{P}(x)$. By the hypotheses, $\left|x^{G}\right|_{p}=\left|G: \mathbf{C}_{G}(x)\right|_{p}=\left|P: P_{0}\right|$ is not divisible by $p^{2}$, so it follows either $\left|P: P_{0}\right|=1$ or $\left|P: P_{0}\right|=p$. The first case yields $P=\mathbf{C}_{P}(x)$ and then $x \in \mathbf{C}_{N}(Z)=1$, a contradiction. Therefore, we may assume that $\left|P: P_{0}\right|=p$, and so $P_{0}$ is normal in $P$. In addition, since $\mathbf{O}_{p}(S) \cap \mathbf{Z}(P)$ is abelian, by the minimality of $Z$, we have either $P_{0} \cap Z=1$ or $P_{0} \cap Z=Z$. The last case gives $Z \leqslant \mathbf{C}_{P}(x)$, a contradiction again. Hence, $P_{0} \cap Z=1$ and it follows that $P=P_{0} \times Z$ and $|Z|=\left|P: P_{0}\right|=p$. We only need to see that $Z=\mathbf{O}_{p}(S)$ to finish the proof.

Note that $\mathbf{Z}(P)=\mathbf{Z}(P) \cap P_{0} Z=Z\left(\mathbf{Z}(P) \cap P_{0}\right)$, so it follows $\mathbf{Z}(P) \cap \mathbf{O}_{p}(S)=Z(\mathbf{Z}(P) \cap$ $\left.P_{0}\right) \cap \mathbf{O}_{p}(S)=Z\left(\mathbf{Z}(P) \cap P_{0} \cap \mathbf{O}_{p}(S)\right)$. If $\mathbf{Z}(P) \cap P_{0} \cap \mathbf{O}_{p}(S) \neq 1$, since it is normal in $\mathbf{O}_{p}(S) \cap \mathbf{Z}(P)$, we can choose a minimal normal subgroup $Z_{1}$ of $\mathbf{O}_{p}(S) \cap \mathbf{Z}(P)$ such that $Z_{1} \leqslant \mathbf{Z}(P) \cap P_{0} \cap \mathbf{O}_{p}(S)$. But then $Z_{1} \leqslant P_{0}=\mathbf{C}_{P}(x)$, so $x \in \mathbf{C}_{N}\left(Z_{1}\right)=1$, a contradiction. Therefore, we may assume that $\mathbf{Z}(P) \cap P_{0} \cap \mathbf{O}_{p}(S)=1$. On the other hand, we have $\mathbf{O}_{p}(S)=$ $\mathbf{O}_{p}(S) \cap Z P_{0}=Z\left(P_{0} \cap \mathbf{O}_{p}(S)\right)$. If $P_{0} \cap \mathbf{O}_{p}(S)$ is a non-trivial subgroup of $P$, since it is normal in $P$, we have a contradiction with $\mathbf{Z}(P) \cap P_{0} \cap \mathbf{O}_{p}(S)=1$. Consequently we get the final contradiction $Z=\mathbf{O}_{p}(S)$. The first assertion is then established.

(2) Let $K / N \mathbf{O}_{p}(G)=\mathbf{O}_{p}\left(G / N \mathbf{O}_{p}(G)\right)$, which has order $p$, and let $P$ be a Sylow $p$ subgroup of $K$. Then $K=P N$. Moreover, $[K, N]$ is normal in $G$ and $[K, N]=[P, N] \leqslant N$ so, by the minimality of $N$, we have either $[P, N]=1$ or $[P, N]=N$. The first case leads to $K=P \times N$, and then $P \leqslant \mathbf{O}_{p}(G)$, a contradiction. Thus we have $[P, N]=N$, and by coprime action it follows $\mathbf{C}_{N}(P)=1$.

Note that every dihedral group of order $2 q$ (for $q$ an odd prime) satisfies the hypotheses of the above proposition (take $p=2$ ).

Theorem $B(3)$ is indeed an immediate consequence of the next more general result.

Theorem 3. Let $G=A B$ be a soluble group, which is the mutually permutable product of the subgroups $A$ and $B$. Assume that $G$ is p-nilpotent for a prime $p$. If $p^{2}$ does not divide $\left|x^{G}\right|$ for any p-regular element $x \in A \cup B$ of prime power order, then $G / \mathrm{O}_{p}(G)$ has elementary abelian Sylow p-subgroups.

Proof. Suppose that the result is false and let $G$ be a minimal counterexample. We may assume by the minimality of $G$ that $\mathbf{O}_{p}(G)=1$, and therefore $\boldsymbol{\Phi}(G)=1$ too. By Theorem 1 , we can assume that there exists a minimal normal subgroup $N$ of $G$ such that $N \leqslant A$. Moreover, $N$ is $q$-elementary abelian, for some prime $q \neq p$. Furthermore, since $N \cap \Phi(G)=1$, by Gaschütz's lemma we may write $G=S N$, with $S \cap N=1$. Let $P$ be a Sylow $p$-subgroup of $S$ (so it is a Sylow $p$-subgroup of $G$ ). Let $T=\mathrm{O}_{p}(S)$. By the minimality of $G$ we have $T \cong \mathbf{O}_{p}(G / N) \neq 1$, and by Proposition 1 (1) it holds $|T|=p$. We may choose $1 \neq x \in N$ such that $P_{0}=\mathbf{C}_{P}(x)$ is a Sylow $p$-subgroup of $\mathbf{C}_{G}(x)$. Since $\mathbf{C}_{N}(T)=1$ by Proposition 1 (2), it holds that $P_{0} \neq P,\left|P: P_{0}\right|=p$, and $P_{0} \cap T=1$. Hence $P=P_{0} \times T$. Finally, since $P_{0} \cong P / T \cong(P N / N) / \mathrm{O}_{p}(G / N)$, which is elementary abelian by the minimality of $G$, it follows that $P$ so is, and this leads to the final contradiction.

Proof of Theorem $\mathbf{B}$. Note that the quotients of $G$ satisfy the hypotheses. Moreover, if $N$ is a normal subgroup of $G$ such that $N=(N \cap A)(N \cap B)$, then $N$ also inherits the hypotheses. (Observe that this occurs, for instance, if either $N \leqslant A$ or $N \leqslant B$.)

(1) We first see that $G$ is soluble by induction over $|G|$. Since every group of odd order is soluble, we may affirm that $p=2$ because $\operatorname{gcd}(p-1,|G|)=1$. By Theorem 11, we can assume 
that there exists a normal subgroup $M$ of $G$ such that $1 \neq M \leqslant A$. If $M<G$, then $M$ is soluble by minimality. Analogously $G / M$ is also soluble, and then so is $G$. If $M=G$, then we apply Theorem 2 .

(2) Suppose that the result is false and let $G$ be a counterexample of minimal order. Since the quotients of $G$ inherit the hypotheses, the class of $p$-nilpotent groups is a saturated formation, and $G$ is soluble, we may assume that $G$ possesses a unique minimal normal subgroup $N$, with $N=\mathbf{C}_{G}(N)=\mathbf{F}(G)$. If $N$ is a $p^{\prime}$-group, since $G / N$ is $p$-nilpotent by the minimality of $G$, it follows that $G$ is $p$-nilpotent, which is a contradiction. Thus, we may assume that $N=\mathbf{O}_{p}(G)$. By Theorem 11, we can assume without loss of generality that $N \leqslant A$, and that there exists a minimal normal subgroup $E / N$ of $G / N$ such that either $E / N \leq A / N$ or $E / N \leqslant B N / N$. In the first case, we have $E \leqslant A$. In the second case, it follows $E=E \cap B N=N(E \cap B) \leqslant$ $(E \cap A)(E \cap B) \leqslant E$. Therefore, we have $E=(E \cap A)(E \cap B)$, where the factors are mutually permutable by Lemma 3 (b). In both cases, $E$ is normal in $G$ and $E$ satisfies the hypotheses. Hence, if $E<G$, then $E$ is $p$-nilpotent by the minimality of $G$. Since $N=\mathrm{O}_{p}(G)$, we get that $E / N$ is $q$-elementary abelian for some prime $q \neq p$, so it follows that $E=Q N$, with $Q$ the normal Sylow $q$-subgroup of $E$. Hence, $Q$ is normal in $G$ which implies that $E=N$, a contradiction.

Therefore, we can assume that $E=G$. So we have $G=E=N Q$, where $Q$ is an abelian Sylow $q$-subgroup of $G$. By Lemma2, we may assume that $Q=(Q \cap A)(Q \cap B)$, with either $Q \cap A \neq 1$ or $Q \cap B \neq 1$. Suppose first that $Q \cap B \neq 1$, and take $1 \neq x \in Q \cap B$. Let $E_{1}=\langle x\rangle N$, which is normal in $Q N=G$. Hence, we have $E_{1}=\langle x\rangle N \leqslant\left(E_{1} \cap B\right)\left(E_{1} \cap A\right) \leqslant E_{1}$. If $Q \cap B=1$, then $G=A$ and we can choose $1 \neq x \in Q$, so that $E_{1}=\langle x\rangle N$ is normal in $Q N=G$. Thus, in both cases, we have that $E_{1}$ inherits the hypotheses and, if $E_{1}<G$, it follows that it is $p$-nilpotent. Therefore $\langle x\rangle$ is a normal Sylow $q$-subgroup of $E_{1}$, which is again a contradiction. Consequently, we may assume that $G=E_{1}=\langle x\rangle N$, for some $q$-element $x$.

Note that $\mathbf{C}_{N}(x)$ is normal in $G=\langle x\rangle N$, since $N$ is abelian. By the minimality of $N$, it follows that either $\mathbf{C}_{N}(x)=1$ or $\mathbf{C}_{N}(x)=N$. The second case leads to $x \in \mathbf{C}_{G}(N)=N$, a contradiction. Hence, it follows that $\mathbf{C}_{G}(x)=\mathbf{C}_{G}(x) \cap N\langle x\rangle=\langle x\rangle \mathbf{C}_{N}(x)=\langle x\rangle$. Then $\left|x^{G}\right|=\left|G: \mathbf{C}_{G}(x)\right|=|N\langle x\rangle:\langle x\rangle|=|N|$, and so $|N|=p$, by the hypotheses. Now, we get that $\langle x\rangle \cong G / N=\mathbf{N}_{G}(N) / \mathbf{C}_{G}(N)$ is isomorphic to a subgroup of $\operatorname{Aut}(N) \cong C_{p-1}$, the cyclic group of order $p-1$. Hence, $|\langle x\rangle|$ divides both $p-1$ and $|G|$, which contradicts the fact that $\operatorname{gcd}(p-1,|G|)=1$. This finishes the proof of the $p$-nilpotency of $G$.

(3) It follows from Theorem 3 .

In the particular case when $G=A=B$ we recover:

Corollary 1. ([13, Theorem A]) Let $G$ be a group. For a fixed prime $p$ with $\operatorname{gcd}(p-1,|G|)=1$, if $p^{2}$ does not divide $\left|x^{G}\right|$ for any p-regular element $x \in G$ of prime power order, then $G$ is soluble, p-nilpotent and $G / \mathbf{O}_{p}(G)$ has elementary abelian Sylow p-subgroups.

Note that if $G$ is the direct product of two symmetric groups of degree 3, then $G$ satisfies the hypotheses of Theorem $\mathrm{B}$ for $p=2$, but not those of Corollary 1 . Moreover, the assumption that $\operatorname{gcd}(p-1,|G|)=1$ is necessary, which can be seen by considering $G=A_{5}$, the alternating group of degree 5 , and the prime $p=3$.

We include here a theorem due to Cossey and Wang $[\mathbf{6}]$, which was the initial motivation for our results, to notify a gap that we have found in one of the statements.

Theorem 4. ([6. Theorem 1]) Let $G$ be a finite group, and $p$ be a prime divisor of $|G|$ such that if $q$ is any prime divisor of $|G|$, then $q$ does not divide $p-1$. Suppose that no conjugacy class size of $G$ is divisible by $p^{2}$. Then $G$ is a soluble p-nilpotent group, and $G / \mathbf{O}_{p}(G)$ has a Sylow p-subgroup of order at most $p$. Further, if $P$ is a Sylow p-subgroup of $G$, then $P^{\prime}$ has order at most $p$, and if $P \neq \mathbf{O}_{p}(G)$, then $\mathbf{O}_{p}(G)$ is abelian. 
Remark 1. (a) The statement " $G / \mathbf{O}_{p}(G)$ has a Sylow $p$-subgroup of order at most $p$ " in the above theorem (and so the corresponding one in [12, Theorem 6]) is not true.

To see this, consider the semidirect product $G=\left[C_{5} \times C_{5}\right]\left(\operatorname{Sym}(3) \times C_{2}\right)($ where $\operatorname{Sym}(3)$ is a symmetric group of degree 3), which is the group of the Small groups library of GAP with identification number $300 \# 25$, and the prime $p=2$. Then $G$ satisfies the hypotheses of Theorem 4 but $\mathbf{O}_{2}(G)=1$ and $|G|_{2}=4$. We reveal that this example has been communicated to us by John Cossey.

(b) The same example shows that the hypotheses in Theorem $B$ for the conjugacy class sizes of the elements $x \in A \cup B$ are not necessarily inherited by the factors, unless they are (sub)normal in $G$. The above group $G$ can be factorised as the mutually permutable product of $A=D_{10} \times D_{10}$ and $B=\left[C_{5} \times C_{5}\right] C_{3}$ (we checked this using GAP). It is clear that $G=A B$ satisfies the hypotheses of Theorem $\mathrm{B}$ for $p=2$, but there are elements $x \in A$ with $\left|x^{A}\right|$ divisible by 4 .

Remark 2. A natural question is how to extend the last assertion of Theorem 4 for (mutually permutable) products. Concerning this, we show the following example:

Let $A=D_{8}$ be a dihedral group of order 8 and $B=\left[C_{5}\right] C_{4}=\langle a, b| a^{5}=b^{4}=1, a^{b}=$ $\left.a^{4}\right\rangle$, and consider the prime $p=2$. Then $G=A \times B$ is a mutually permutable product of $A$ and $B$, and $G$ is 2-nilpotent. Moreover, 4 does not divide any conjugacy class size of elements in $A \cup B$. However, $\mathbf{O}_{2}(G)=\left(\mathbf{O}_{2}(G) \cap A\right)\left(\mathbf{O}_{2}(G) \cap B\right)=D_{8} \times C_{2}$ is not abelian.

Regarding the claim " $P^{\prime}$ has order at most $p$ " in Theorem 4, we get the next extension for factorised groups, as an immediate consequence of Theorem A.

Corollary 2. Let $G=A B$ be the product of the subgroups $A$ and $B$. Assume that $G$ is $p$ nilpotent, and that for all p-elements in the factors, $p^{2}$ does not divide $\left|x^{G}\right|$. If $P$ is a Sylow p-subgroup of $G$, then $P^{\prime} \leqslant \boldsymbol{\Phi}(P) \leqslant \mathbf{Z}(P)$, with $P^{\prime}$ elementary abelian of order at most $p^{2}$.

In particular, from this fact and Theorem $B$, we get [12, Theorem 7] as a corollary, taking $G=A=B$.

Finally, we prove Theorem C, which is motivated by [2, Theorem 1.3].

Proof of Theorem C. Suppose that the result is false and let $G$ be a counterexample of minimal order. Note that $G$ cannot be simple. Since the class of $p$-supersoluble groups is a saturated formation, we may assume that there exists a unique minimal normal subgroup $N$ of $G$, and that $\Phi(G)=1$. By the minimality of $G$, we get that $G / N$ is $p$-supersoluble. Since $G$ is $p$-soluble, it follows that $N$ is either a $p$-group or a $p^{\prime}$-group. In the second case, since $G / N$ satisfies the thesis by minimality, we get a contradiction. Consequently, we may assume that $N$ is $p$-elementary abelian and we must show that $|N|=p$. As $\boldsymbol{\Phi}(G)=1$ and $G$ is $p$-soluble with $\mathbf{O}_{p^{\prime}}(G)=1$, by [7. A - 10.6] it follows that $\mathbf{F}(G)=\operatorname{Soc}(G)=N=\mathbf{C}_{G}(N)$, and also $N=\mathbf{O}_{p}(G)$. Applying Theorem 1, we may assume that there exists a minimal normal subgroup $Z / N$ of $G / N$ such that $Z / N \leqslant A N / N$, so $Z=Z \cap A N=N(Z \cap A)$. Since $G / N$ is $p$-soluble, it follows that $Z / N$ is either a $p$-group or a $p^{\prime}$-group. The first case leads to $Z / N \leqslant \mathbf{O}_{p}(G / N)=\mathbf{O}_{p}\left(G / \mathbf{O}_{p}(G)\right)=1$, a contradiction. Hence, we may assume that $Z / N$ is a $p^{\prime}$-group.

Let $Q$ be a Sylow $q$-subgroup of $Z \cap A$, where $q \neq p$ is a prime (so $Q$ is a Sylow $q$-subgroup of $Z$ ). Therefore $Q \cong Q N / N$ is a Sylow $q$-subgroup of $Z / N$, which acts faithfully on $N$. If $1 \neq a \in Q \leqslant A$, then $N=[N, a] \times \mathbf{C}_{N}(a)$. By the hypotheses, since $p^{2}$ does not divide $\left|a^{G}\right|=\left|G: \mathbf{C}_{G}(a)\right|$, then neither divides $\left|N: \mathbf{C}_{N}(a)\right|=|[N, a]|$, so either $|[N, a]|=1$ or $|[N, a]|=p$. The first case leads to $a \in \mathbf{C}_{G}(N)=N$, a contradiction. Thus, Lemma 4 yields $Q N / N$ is cyclic. Since this is valid for all primes $q \neq p$, we get by $[\mathbf{9}, 5.15]$ that $Z / N$ is soluble. By the minimality of $Z / N$, it follows that $(Z / N)^{\prime}=1$ and $Z / N$ is abelian with cyclic Sylow 
subgroups. Consequently $Z / N=\langle x N\rangle$, where $x \notin N$ and the order of $x N$ is $q$, for some prime $q \neq p$.

We may assume that $x \in Z \cap A$ and $Z=N\langle x\rangle$. Hence $\mathbf{C}_{N}(x)=\mathbf{C}_{N}(Z)$. By the minimality of $N$, we have either $\mathbf{C}_{N}(Z)=N$ or $\mathbf{C}_{N}(Z)=1$. The first case leads to $x \in Z \leqslant \mathbf{C}_{G}(N)=N$, a contradiction. Therefore, since $N=[N, x] \times \mathbf{C}_{N}(x)$, it follows $|N|=|[N, x]|=p$, and this final contradiction establishes the theorem.

Example 3. Let $G$ be the symmetric group of degree 4 . Then $G=A B$ is a mutually permutable product, where $A$ denotes the alternating group of degree 4 and $B$ is a Sylow 2-subgroup of $G$, which satisfies the hypotheses of Theorem C, for $p=3$.

\section{Square-free class sizes}

We begin this section with the proof of Theorem $D$

Proof of Theorem D. (1) Suppose that the result is false and let $G$ be a counterexample of least possible order. Since $G$ supersoluble, $G / \mathbf{F}(G)$ is abelian, and so $G^{\prime} \leqslant \mathbf{F}(G)$. Moreover, the quotients of $G$ inherit the hypotheses and the class of metabelian groups is a formation, so we may assume that there exists a unique minimal normal subgroup $N$ of $G$ with $|N|=p$, for some prime divisor $p$ of $|G|$. Hence, $\mathbf{F}(G)=\mathrm{O}_{p}(G) \leqslant P$, a Sylow $p$-subgroup of $G$. Since $G / \mathbf{F}(G)$ is abelian, $P=\mathbf{O}_{p}(G)=\mathbf{F}(G)$. Hence $P=(P \cap A)(P \cap B)$, where $P \cap A$ and $P \cap B$ are Sylow $p$-subgroups of $A$ and $B$ respectively, by Lemma2 Applying Theorem A, we have $P^{\prime} \leqslant \mathbf{\Phi}(P) \leqslant \mathbf{Z}(P)$, and $P^{\prime}$ is elementary abelian of order at most $p^{2}$. Note that $P^{\prime} \neq 1$, because $G^{\prime} \leq P$.

By Lemma2, we may consider $H$ a Hall $p^{\prime}$-subgroup of $G$, such that $H=(H \cap A)(H \cap B)$, where $H \cap A$ and $H \cap B$ are Hall $p^{\prime}$-subgroups of $A$ and $B$ respectively. Moreover, $H \cong G / \mathbf{F}(G)$ is abelian. Let $x \in H \cap A$ be a prime power order element. Since $H \leqslant \mathbf{C}_{G}(x) \leqslant G$, it follows by the hypotheses that $\left|x^{G}\right| \leq p$, and so $\boldsymbol{\Phi}(G) \leqslant \mathbf{C}_{G}(x)$. Thus $\boldsymbol{\Phi}(G) \leqslant \mathbf{C}_{G}(H \cap A)$, and analogously for $H \cap B$. Consequently we get $P^{\prime} \leqslant \boldsymbol{\Phi}(P) \leqslant \boldsymbol{\Phi}(G) \leqslant \mathbf{C}_{G}(H)$. Since $P^{\prime} \leqslant \boldsymbol{\Phi}(P) \leqslant \mathbf{Z}(P)$, it follows $P^{\prime} \leqslant \boldsymbol{\Phi}(P) \leqslant \mathbf{Z}(G)$. In particular, $\boldsymbol{\Phi}(G) \neq 1 \neq \mathbf{Z}(G)$.

If $A, B \leqslant P$, then $G=P$ and $G^{\prime}=P^{\prime} \leqslant \mathbf{Z}(G)$, a contradiction. Hence we have either $H \cap A \neq 1$ or $H \cap B \neq 1$. Assume $H \cap A \neq 1$. Let $Q_{A}$ be a Sylow $q$-subgroup of $H \cap A$, for some prime $q \neq p$. Note that $Q_{A} \nless \mathbf{Z}(G)$, because $\mathbf{Z}(G)$ is a $p$-group. Let $\overline{Q_{A}}=Q_{A} \mathbf{Z}(G) / \mathbf{Z}(G)$, which acts on $\bar{P}=P / \mathbf{Z}(G)$, which is elementary abelian because $\boldsymbol{\Phi}(P) \leqslant \mathbf{Z}(G) \leqslant \mathbf{F}(G)=P$. Suppose $\bar{w}=w \mathbf{Z}(G) \in \mathbf{C}_{\overline{Q_{A}}}(\bar{P})$. Then $[\bar{w}, \bar{y}]=1$ for all $\bar{y}=y \mathbf{Z}(G) \in \bar{P}$, so $[w, y] \in \mathbf{Z}(G) \leqslant$ $P$. Let $k=o(w)$ denote the order of $w$. Thus $[w, y]^{k}=\left[w^{k}, y\right]=1$. It follows $[w, y]=1$ for all $y \in P$, so $w \in \mathbf{C}_{Q_{A}}(P)=\mathbf{C}_{Q_{A}}(\mathbf{F}(G))=1$. Then $\mathbf{C}_{\overline{Q_{A}}}(\bar{P})=1$ and the action is faithful. Let $1 \neq \overline{\alpha_{q}}=\alpha_{q} \mathbf{Z}(G) \in \overline{Q_{A}}$. Therefore $\bar{P}=\left[\bar{P}, \overline{\alpha_{q}}\right] \times \mathbf{C}_{\bar{P}}\left(\overline{\alpha_{q}}\right)$, with $\left[\bar{P}, \overline{\alpha_{q}}\right] \neq 1$. Moreover, $\left|\left[\bar{P}, \overline{\alpha_{q}}\right]\right|=\left|\bar{P}: \mathbf{C}_{\bar{P}}\left(\overline{\alpha_{q}}\right)\right|=\left|\bar{P}: \overline{\mathbf{C}_{P}\left(\alpha_{q}\right)}\right|=\left|P: \mathbf{C}_{P}\left(\alpha_{q}\right)\right|$, which divides $\left|\alpha_{q}^{G}\right|$, because $P$ is normal in $G$. Since $H \leqslant \mathbf{C}_{G}\left(\alpha_{q}\right)$ and $\alpha_{q}$ is a non-central prime power order element in $A$, it follows $\left|\alpha_{q}^{G}\right|=p$, and so $\left|\left[\bar{P}, \overline{\alpha_{q}}\right]\right|=p$. Applying Lemma 4 , we get that $\overline{Q_{A}} \cong Q_{A}$ is cyclic. Since this is valid for each prime divisor $q$ of $|H \cap A|$, we deduce that $H \cap A$ has cyclic Sylow subgroups, but it is abelian, so $H \cap A$ is cyclic. Analogously, if $H \cap B \neq 1$, then it is cyclic.

Let $H \cap A=\langle\alpha\rangle$. Assume first that $1 \neq \alpha$ is a $q$-element, for some prime $q$, and that $G=P\langle\alpha\rangle$. By the above argument, $\langle\alpha\rangle \mathbf{Z}(G) / \mathbf{Z}(G)$ acts faithfully on $\bar{P}$, and $|[\bar{P}, \bar{\alpha}]|=$ $\left|P: \mathbf{C}_{P}(\alpha)\right|=p$. Let $y \in P \backslash \mathbf{C}_{P}(\alpha)$. If $[\bar{y}, \bar{\alpha}]=1$, then $[y, \alpha] \in \mathbf{Z}(G) \leqslant P$. Hence $[y, \alpha]^{o(\alpha)}=\left[y, \alpha^{o(\alpha)}\right]=1$, so $[y, \alpha]=1$ and $y \in \mathbf{C}_{P}(\alpha)$, a contradiction. Then $[\bar{y}, \bar{\alpha}] \neq 1$, and since $|[\bar{P}, \bar{\alpha}]|=p$, it follows $[\bar{P}, \bar{\alpha}]=\langle[\bar{y}, \bar{\alpha}]\rangle$. Therefore we have $\overline{[P, \alpha]}=[\bar{P}, \bar{\alpha}]=\langle[\bar{y}, \bar{\alpha}]\rangle=$ $\overline{\langle[y, \alpha]\rangle}$, so $[P, \alpha] \leqslant[P, \alpha] \mathbf{Z}(G)=\langle[y, \alpha]\rangle \mathbf{Z}(G)$, and then $G^{\prime}=P^{\prime}[P, \alpha] \leqslant\langle[y, \alpha]\rangle \mathbf{Z}(G)$ which is abelian, a contradiction. 
Hence, we may assume that, for every prime $q$, if $\alpha_{q}$ is the $q$-part of $\alpha$, then $P\left\langle\alpha_{q}\right\rangle<G$. Note that $P\left\langle\alpha_{q}\right\rangle$ is normal in $G$, and $P\left\langle\alpha_{q}\right\rangle=\left(P\left\langle\alpha_{q}\right\rangle \cap A\right)\left(P\left\langle\alpha_{q}\right\rangle \cap B\right)$. Therefore, by the minimality of $G$, it follows that $\left(P\left\langle\alpha_{q}\right\rangle\right)^{\prime}$ is abelian. Notice that $\left(P\left\langle\alpha_{q}\right\rangle\right)^{\prime}=P^{\prime}\left[P, \alpha_{q}\right]$, since $P$ is normal in $G$. Let $K=P^{\prime}\left[P, \alpha_{q}\right]$, which is an abelian normal subgroup of $G$, and let $t \in\left[P, \alpha_{q}\right] \leqslant K$. Then Lemma 5 leads to $\left|\mathbf{C}_{G}\left(\alpha_{q}\right)\right|<\left|\mathbf{C}_{G}\left(\left[t, \alpha_{q}\right]\right)\right|$. If $p$ divides $\left|\left[t, \alpha_{q}\right]^{G}\right|$ we get a contradiction, because $\left|\left[t, \alpha_{q}\right]^{G}\right|<\left|\alpha_{q}^{G}\right|=p$. Hence, $P \leqslant \mathbf{C}_{G}\left(\left[t, \alpha_{q}\right]\right)$ and $\left[t, \alpha_{q}\right] \in \mathbf{Z}(P)$, for each $t \in\left[P, \alpha_{q}\right]$. By coprime action, $P=\left[P, \alpha_{q}\right] \mathbf{C}_{P}\left(\alpha_{q}\right)$. Thus $\left[P, \alpha_{q}\right]=$ $\left[\left[P, \alpha_{q}\right] \mathbf{C}_{P}\left(\alpha_{q}\right), \alpha_{q}\right]=\left[P, \alpha_{q}, \alpha_{q}\right]$. If $k$ is a generator of $\left[P, \alpha_{q}, \alpha_{q}\right]$, then $k=\left[t, \alpha_{q}\right] \in \mathbf{Z}(P)$ with $t \in\left[P, \alpha_{q}\right]$, so $\left[P, \alpha_{q}\right]=\left[P, \alpha_{q}, \alpha_{q}\right] \leqslant \mathbf{Z}(P)$. Since this is valid for each prime divisor $q$ of the order of $H \cap A=\langle\alpha\rangle$, we get:

$$
[P, H \cap A]=\left[P,\left\langle\alpha_{q_{1}}\right\rangle \times \cdots \times\left\langle\alpha_{q_{t}}\right\rangle\right]=\left[P, \alpha_{q_{1}}\right] \cdots\left[P, \alpha_{q_{t}}\right] \leqslant \mathbf{Z}(P) .
$$

Analogously, if $H \cap B \neq 1$, then $[P, H \cap B] \leq \mathbf{Z}(P)$. Since $G^{\prime}=P^{\prime}[P, H]=P^{\prime}[P, H \cap$ $A][P, H \cap B]$, we get $G^{\prime} \leq \mathbf{Z}(P)$. This final contradiction establishes statement (1).

(2) Suppose that the second assertion is not true and let $G$ be a counterexample of minimal order. We point out that the hypotheses are inherited by every quotient group of $G$ and, by (1), $G^{\prime}$ is abelian. There exists a prime divisor $p$ of $\left|G^{\prime}\right|$ such that $G^{\prime}$ does not have any elementary abelian Sylow $p$-subgroup. By the minimality of $G$, we may consider that $\mathbf{O}_{p^{\prime}}(G)=1$. Moreover, since $G$ is supersoluble, then $G / \mathbf{F}(G)$ is abelian, and $\mathbf{F}(G)=\mathbf{O}_{p}(G)=P$ is a normal Sylow $p$-subgroup of $G$ such that $G^{\prime} \leqslant P$. Using Lemma 2 and Theorem A, we obtain respectively that $P=(P \cap A)(P \cap B)$, and that $P^{\prime}$ is elementary abelian with $P^{\prime} \leqslant \mathbf{\Phi}(P) \leqslant \mathbf{Z}(P)$.

Let $G^{\mathfrak{N}}$ be the nilpotent residual of $G$. Note that $G^{\mathfrak{N}} \neq 1$; in other case, $G$ is a $p$-group and then $G^{\prime}=P^{\prime}$, a contradiction. Since $G^{\mathfrak{N}} \leqslant G^{\prime}$, it follows that $G^{\mathfrak{N}}$ is abelian. By using [7, III - 4.6, IV - 5.18], we have that $G^{\mathfrak{N}}$ is complemented in $G$, and its complements are precisely the Carter subgroups of $G$. Accordingly, $G=G^{\mathfrak{N}} H$ with $H=\mathbf{N}_{G}(H)$ a nilpotent subgroup of $G$ and $G^{\mathfrak{N}} \cap H=1$. These facts yield $G^{\prime}=G^{\mathfrak{N}} \times\left(H \cap G^{\prime}\right)$, and $\mathbf{C}_{G^{\mathfrak{N}}}(H)=1$. On the other hand, the minimality of $G$ implies that $G^{\prime} / G^{\mathfrak{N}} \cong\left(H \cap G^{\prime}\right)$ is elementary abelian, and thus $G^{\mathfrak{N}}$ is not so. If $\mathbf{C}_{H}\left(G^{\mathfrak{N}}\right) \neq 1$, since $\mathbf{C}_{H}\left(G^{\mathfrak{N}}\right)$ is normal in $G$, by the minimality of $G$ we have $\left(G / \mathbf{C}_{H}\left(G^{\mathfrak{N}}\right)\right)^{\prime}$ is an elementary abelian group, but

$$
\left(G / \mathbf{C}_{H}\left(G^{\mathfrak{N}}\right)\right)^{\prime}=G^{\prime} \mathbf{C}_{H}\left(G^{\mathfrak{N}}\right) / \mathbf{C}_{H}\left(G^{\mathfrak{N}}\right)=G^{\mathfrak{N}} \mathbf{C}_{H}\left(G^{\mathfrak{N}}\right) / \mathbf{C}_{H}\left(G^{\mathfrak{N}}\right) \cong G^{\mathfrak{N}},
$$

which is a contradiction. Hence, $\mathbf{C}_{H}\left(G^{\mathfrak{N}}\right)=1$. In particular, we deduce that $\mathbf{Z}(G)=1$.

By Lemma 2, there exists a Hall $p^{\prime}$-subgroup $H_{0}$ of $G$ such that $H_{0}=\left(H_{0} \cap A\right)\left(H_{0} \cap B\right)$. Let $x \in H_{0} \cap A$ be a non-trivial element of prime power order. Since $H_{0} \leqslant \mathbf{C}_{G}(x)$, it follows that $\left|x^{G}\right| \leq p$, and so $\boldsymbol{\Phi}(G) \leqslant \mathbf{C}_{G}(x)$. Thus $\boldsymbol{\Phi}(G) \leqslant \mathbf{C}_{G}\left(H_{0} \cap A\right)$, and analogously for $H_{0} \cap B$. Consequently, we get $P^{\prime} \leqslant \boldsymbol{\Phi}(P) \leqslant \boldsymbol{\Phi}(G) \leqslant \mathbf{C}_{G}\left(H_{0}\right)$. Since $P^{\prime} \leqslant \boldsymbol{\Phi}(P) \leqslant \mathbf{Z}(P)$ and $G=P H_{0}$, it follows $P^{\prime} \leqslant \mathbf{\Phi}(P) \leqslant \mathbf{Z}(G)=1$, which implies that $P$ is elementary abelian, the final contradiction.

(3) Assume that the result is false and take $G$ a counterexample of minimal order. Consider a prime $p$ such that $\left|\mathbf{F}(G)^{\prime}\right|_{p}>p^{2}$. By minimality, we can affirm that $\mathbf{O}_{p^{\prime}}(\mathbf{F}(G))=1$. Since $G$ is supersoluble, we get that $\mathbf{F}(G)=\mathbf{O}_{p}(G)=P$ is a normal Sylow $p$-subgroup of $G$. Then, we apply both Lemma 2 and Theorem A to get the final contradiction.

Example 4. Let $G=A \times B$ be the direct product of two symmetric groups of degree 3. Then $G$ is supersoluble, and every element contained in each factor (not only those of prime power order) has square-free conjugacy class size, but neither the derived subgroup $G^{\prime}$ nor $G / \mathbf{F}(G)$ are cyclic, in contrast to [6, Theorem 2]. 
Example 5. In view of [6. Theorem 2], it is natural to wonder if we can affirm in the above result that the Sylow $p$-subgroups of $G^{\prime}$ have order at most $p^{2}$. This fact is not further true, as we show:

Let $G=A \times B$, where $A=D_{14}$ is a dihedral group of order 14 , and $B=D_{14} \times\left[C_{7}\right] C_{3}$ is the direct product of such a dihedral group and a semidirect product of a cyclic group of order 7 and a cyclic group of order 3 ( $B$ has identification number $294 \# 9$ in the Small Groups library of GAP). Then $G$ is supersoluble, and it satisfies that all prime power order elements contained in each factor have square-free conjugacy class size, but $G^{\prime}$ has order $7^{3}$.

Now we proceed with the proof of Theorem $E$

Proof of Theorem E. Considering the smallest prime divisor of $|G|$ and Theorem $\mathrm{B}$, we conclude that $G$ is soluble. Hence, it is $p$-soluble for each prime divisor $p$ of $|G|$. Applying Theorem C. we get that $G$ is $p$-supersoluble for each prime that divides $|G|$, so it is supersoluble.

Now we prove the second assertion by induction on $|G|$. Let $p$ be an arbitrary prime, and $P$ be a Sylow $p$-subgroup of $G$. We want to show that $P \mathbf{F}(G) / \mathbf{F}(G) \cong P / \mathbf{O}_{p}(G)$ is elementary abelian. Since $G$ is supersoluble, we have that $G / \mathbf{F}(G)$ is abelian. Moreover, we may assume by induction that $\mathbf{O}_{p}(G)=1$. Therefore, we have that $\mathbf{F}(G) \leqslant H \leqslant G$, where $H$ is a Hall $p^{\prime}$-subgroup of $G$. Consequently, $H$ is normal in $G$ and $G$ is $p$-nilpotent. Finally, by Theorem 3 the result is established.

When considering in the above theorem all $p$-regular elements in the factors, we get as a corollary:

Corollary 3. ([2, Corollary 1.5]) Let the group $G=A B$ be the mutually permutable product of the subgroups $A$ and $B$. Suppose that for every prime $p$ and every p-regular element $x \in A \cup B$, $\left|x^{G}\right|$ is not divisible by $p^{2}$. Then $G$ is supersoluble.

Example 6. Consider $G=A \times B$, where $A=\operatorname{Sym}(3)$ is a symmetric group of degree 3, and $B=\operatorname{Sym}(3) \times D_{10}$ is the direct product of such a symmetric group and a dihedral group of order 10. Then $G$ satisfies the hypotheses of Theorem E. However there exists some 2-regular element in $B$, not of prime power order, such that 4 divides its conjugacy class size, so Corollary 3 cannot be applied.

In the particular case when $A$ and $B$ are normal in $G$, we obtain [12, Proposition 9].

Corollary 4. Let $A$ and $B$ be normal subgroups of $G$ such that $G=A B$. Suppose that $\left|x^{G}\right|$ is square-free for every element $x$ of prime power order of $A \cup B$. Then $G$ is supersoluble.

This development has its origins in the contributions of Chillag and Herzog [5, Theorem 1], and Cossey and Wang [6. Theorem 2]. Our next result Theorem $\mathrm{F}$ and Theorem $\mathrm{D}$ can be considered somehow extensions of the ones above for (mutually permutable) products. In fact, Theorem $\mathrm{F}$ provides further information on the Sylow subgroups of $G / \mathbf{F}(G)$.

Proof of Theorem F. Suppose the result is not true and let $G$ be a counterexample of least order possible. Then if $P$ is a Sylow $p$-subgroup of $G$, we have $\left|P / \mathbf{O}_{p}(G)\right| \geq p^{3}$. We can assume by the minimality of $G$ that $\mathbf{O}_{p}(G)=1=\boldsymbol{\Phi}(G)=\mathbf{Z}(G)$, so $|P| \geq p^{3}$. By Lemma 2 we can choose $P=(P \cap A)(P \cap B)$, with $P \cap A$ and $P \cap B$ Sylow $p$-subgroups of $A$ and $B$ respectively. By Theorem $\mathrm{E}$, we have that $G$ is supersoluble and $G / \mathbf{F}(G)$ has elementary abelian Sylow subgroups. In particular, $P \mathbf{F}(G)$ is normal in $G$. Hence Lemma 3 (b) asserts that $L=(P \mathbf{F}(G) \cap A)(P \mathbf{F}(G) \cap B)$ is normal in $G$, and it is a mutually permutable product. Moreover, $P=(P \cap A)(P \cap B) \leqslant(P \mathbf{F}(G) \cap A)(P \mathbf{F}(G) \cap B)=L \leqslant P \mathbf{F}(G)$. If we suppose $L<G$, by the minimality of $G$ it follows $\left|P / \mathbf{O}_{p}(L)\right|=|P| \leq p^{2}$, a contradiction. Thus, we may assume $L=G=P \mathbf{F}(G)$, and so $G$ is $p$-nilpotent. 
Let $N$ be a minimal normal subgroup of $G$. We can assume without loss of generality that it is contained in $A$ by Theorem 1. Note $|N|=q \neq p$. By Proposition 1 (1), it follows $\left|\mathbf{O}_{p}(G / N)\right| \leq p$, and by the minimality of $G$ we have $\left|(P N / N) / \mathbf{O}_{p}(G / N)\right| \leq p^{2}$. Since $P \cong P N / N$, we may assume $|P|=p^{3}$. As $\Phi(G)=1$, [7, A - 10.6 Theorem] leads to $\mathbf{F}(G)=\operatorname{Soc}(G)=\mathbf{C}_{G}(\mathbf{F}(G))$. If $N$ is the unique minimal normal subgroup of $G$, then $P \cong$ $\mathbf{N}_{G}(N) / \mathbf{C}_{G}(N)$ which is isomorphic to a subgroup of $\operatorname{Aut}\left(C_{q}\right) \cong C_{q-1}$, so $P$ is cyclic and elementary abelian, which implies that its order is $p$, a contradiction.

Now we denote by $T$ a complement of $N$ in $\mathbf{F}(G)$, so $T \neq 1$. It follows $\mathbf{F}(G)=\operatorname{Soc}(G)=$ $N \times T$. We denote $Q_{1}=\mathbf{O}_{p}(P N)$ and $Q_{2}=\mathbf{O}_{p}(P T)$. Since $P N \cong G / T$ (and $\left.P T \cong G / N\right)$, by the minimality of $G$ we may affirm $Q_{1} \neq 1 \neq Q_{2}$. On the other hand, since $\left[\mathbf{O}_{p}(P N), N\right] \leqslant$ $\mathbf{O}_{p}(P N) \cap N=1$, we have $Q_{1} \leqslant C_{1}=\mathbf{C}_{P}(N)$ (analogously $Q_{2} \leqslant C_{2}=\mathbf{C}_{P}(T)$ ). In addition, it follows $C_{1} \cap C_{2} \leqslant \mathbf{C}_{P}(\mathbf{F}(G)) \leqslant \mathbf{C}_{G}(\mathbf{F}(G)) \leqslant \mathbf{F}(G)$, so $C_{1} \cap C_{2}=1$. Let $P_{0}$ be a Sylow $p$ subgroup of $\mathbf{C}_{G}(N)$ such that $P_{0} \leqslant P$. Hence $P_{0}=C_{1}=\mathbf{C}_{P}(N)$. In addition, since $N=\langle x\rangle$ where $x$ is a $q$-element contained in $A$, by the hypotheses it follows that $p^{2}$ does not divide $\left|x^{G}\right|_{p}=\left|G: \mathbf{C}_{G}(x)\right|_{p}=\left|P: C_{1}\right|$. Moreover, since $|P|=\left|P: C_{1}\right| \cdot\left|C_{1}\right|=p^{3}$, we may assume $\left|C_{1}\right| \geq p^{2}$, and since $1 \neq Q_{2} \leqslant C_{2}$, we have $\left|C_{2}\right| \geq p$. Accordingly $\left|C_{1} C_{2}\right|=\left|C_{1}\right| \cdot\left|C_{2}\right| \geq p^{3}$, and since $P$ is abelian, we have necessarily $P=C_{1} \times C_{2}$. This leads to

$$
G=P \mathbf{F}(G)=C_{1} C_{2} N T=\left(C_{1} T\right) \times\left(C_{2} N\right) .
$$

Suppose $T \cap A \neq 1$, and let $1 \neq y \in T \cap A \leqslant C_{1} T \cap A$. Let $1 \neq x \in N \leqslant A$. Then since $x y \in \mathbf{F}(G) \cap A$ and $\mathbf{F}(G)$ is abelian, we have that $x y$ is a $p$-regular element, so by the hypotheses $p^{2}$ does not divide $\left|(x y)^{G}\right|$. As $G$ is a direct product, we have $\left|(x y)^{G}\right|=\left|x^{G}\right| \cdot\left|y^{G}\right|$. In addition, $\left(N \times C_{1} T\right) \leqslant \mathbf{C}_{G}(x) \leqslant G$, and therefore $\left|x^{G}\right|$ divides $\left|G:\left(C_{1} T \times N\right)\right|=\left|C_{2}\right|$ which is a $p$ number, so $\left|x^{G}\right|=p$ (recall that $\mathbf{Z}(G)=1$ ), and analogously $\left|y^{G}\right|=p$, a contradiction. We conclude $T \cap A=1$.

If $\mathbf{F}(G)=K \times M$ with $M$ normal in $G$ and $K$ a minimal normal subgroup of $G$ contained in $B$, by similar arguments we can deduce $M \cap B=1$. This means, in particular, that neither $A$ nor $B$ can contain two distinct minimal normal subgroups of $G$.

On the other hand, since $\mathbf{F}(G)$ is the unique $p^{\prime}$-Hall subgroup of $G$, Lemma 2 leads to $\mathbf{F}(G)=(\mathbf{F}(G) \cap A)(\mathbf{F}(G) \cap B)$. Moreover, since $\mathbf{F}(G) \cap A=N T \cap A=N(T \cap A)=N$, it follows $\mathbf{F}(G)=N(\mathbf{F}(G) \cap B)$. Note that $(\mathbf{F}(G) \cap B) \cap N \leqslant N$ with $|N|=q$ so we distinguish two cases: either $(\mathbf{F}(G) \cap B) \cap N=N$ or $(\mathbf{F}(G) \cap B) \cap N=1$. In the first case $\mathbf{F}(G)=$ $\mathbf{F}(G) \cap B \leqslant B$. Thus there exists another minimal normal subgroup contained in $B$ and distinct of $N$, a contradiction. Hence we conclude $(\mathbf{F}(G) \cap B) \cap N=1$ so $\mathbf{F}(G)=N \times(\mathbf{F}(G) \cap B)$.

Now suppose that $\mathbf{F}(G)$ is a $q$-group. Then, since $\mathbf{F}(G)=\operatorname{Soc}(G)$, it follows that $\mathbf{F}(G)$ is $q$-elementary abelian. In addition, $P \cap A$ acts faithfully over $\mathbf{F}(G)$. Let $1 \neq x \in P \cap A \leqslant A$, then $\mathbf{F}(G)=[\mathbf{F}(G), x] \times \mathbf{C}_{\mathbf{F}(G)}(x)$, with $\mathbf{C}_{\mathbf{F}(G)}(x)<\mathbf{F}(G)$ since $\mathbf{Z}(G)=1$ and $P$ is abelian. By the hypotheses, $q^{2}$ does not divide $\left|x^{G}\right|$, and therefore it does not divide $\left|\mathbf{F}(G): \mathbf{C}_{\mathbf{F}(G)}(x)\right|$. Thus we may affirm $|[\mathbf{F}(G), x]|=q$. By Lemma 4 we conclude that $P \cap A$ is cyclic, and analogously $P \cap B$ is cyclic too. So they are both cyclic and elementary abelian, that is, they both have order $p$. Thus $|P|=|(P \cap A)(P \cap B)| \leq p^{2}$, a contradiction.

Hence we may suppose that there exists a prime $r \neq q$ such that $r$ divides $|\mathbf{F}(G)|$. Let $1 \neq R$ be a Sylow $r$-subgroup of $\mathbf{F}(G) \cap B$ (so it is a Sylow $r$-subgroup of $\mathbf{F}(G)$ ). Then $1 \neq R=$ $\mathbf{O}_{r}(G) \leqslant B$, and since $\mathbf{F}(G)=\operatorname{Soc}(G)$, necessarily we have that $\mathbf{O}_{r}(G)$ is the product of the minimal normal subgroups of $G$ with order $r$. Let $M \leqslant B$ be one of those minimal normal subgroups. Arguing exactly in the same way as with $N$, it follows $\mathbf{F}(G)=M \times(A \cap \mathbf{F}(G))$. But $A \cap \mathbf{F}(G)=N$ so $\mathbf{F}(G)=N \times M$ with both minimal normal subgroups of $G, N \leqslant A$ and $M \leqslant B$. Let $P_{1}$ be a Sylow $p$-subgroup of $\mathbf{C}_{G}(N)=\mathbf{C}_{G}(x)$ such that $P_{1} \leqslant P$. Then by the hypotheses we have $\left|x^{G}\right|_{p}=\left|P: P_{1}\right| \leq p$. Since $|P|=p^{3}$, it follows $\left|P_{1}\right| \geq p^{2}$. 
However, $P_{1}$ is normal in $P N$ so $P_{1} \leqslant \mathbf{O}_{p}(P N) \cong \mathbf{O}_{p}(G / M)$, and by Proposition 11 (1) we have $\left|\mathbf{O}_{p}(G / M)\right| \leq p$. This final contradiction establishes the theorem.

Example 7. Under the hypotheses of Theorem $\mathrm{E}$ (even under those of Theorem $\mathrm{D}$ ), it is not possible to assure that $G / \mathbf{F}(G)$ has Sylow $p$-subgroups of order at most $p^{2}$, as the following example shows:

Let $\left\{p_{1}, p_{2}, \ldots, p_{n}\right\}$ be a finite set of pairwise distinct odd primes, and let $G=D_{2 p_{1}} \times$ $D_{2 p_{2}} \times \cdots \times D_{2 p_{n}}$ be the direct product of dihedral groups of order $2 p_{i}, 1 \leq i \leq n$. Then $G=A \times B$ is a mutually permutable product of $A=D_{2 p_{1}}$ and $B=D_{2 p_{2}} \times \cdots \times D_{2 p_{n}}$, and each prime power order element contained in the direct factors has square-free conjugacy class size. However, $G / \mathbf{F}(G)$ has order $2^{n}$.

\section{References}

[1] B. Amberg, S. Franciosi, And F. de Giovanni. Products of groups. Oxford University Press Inc., 1992.

[2] A. Ballester-Bolinches, J. Cossey, And Y. Li. "Mutually permutable products and conjugacy classes". Monatsh. Math. 170 (2013) 305-310.

[3] A. Ballester-Bolinches, R. Esteban-Romero, And M. AsaAd. Products of finite groups. Vol. 53. W. de Gruyter Expositions In Mathematics, 2010.

[4] J. C. Beidleman And H. HeineKen. "Group classes and mutually permutable products". $J$. Algebra 297 (2006) 409-416.

[5] D. Chillag And M. Herzog. "On the length of the conjugacy classes of finite groups". $J$. Algebra 131 (1990) 110-125.

[6] J. COSSEY AND Y. WANG. "Remarks on the length of conjugacy classes of finite groups". Comm. Algebra 27 (1999) 4347-4353.

[7] K. Doerk And T. HAWKES. Finite Soluble Groups. Vol. 4. W. de Gruyter Expositions In Mathematics, 1992.

[8] B. HupperT. Character Theory of Finite Groups. Vol. 25. W. de Gruyter Expositions In Mathematics, 1998.

[9] I. M. Is ACS. Finite Group Theory. American Mathematical Society, 2008.

[10] H. G. KNOCHE. "Über den Frobeniusschen Klassenbegriff in nilpotenten Gruppen”. Math. Z. 55 (1951) 71-83.

[11] S. LI. "On the class length of elements of prime power order in finite groups". Guangxi Sci. 6 (1999) 12-13.

[12] X. LiU, Y. WANG, AND H. WEI. "Notes on the length of conjugacy classes of finite groups". $J$. Pure Appl. Algebra 196 (2005) 111-117.

[13] G. QIAN AND Y. WANG. "On conjugacy class sizes and character degrees of finite groups". $J$. Algebra Appl. 13 (2014) 1350100 (9 pages).

[14] The GAP Group, GAP - Groups, Algorithms, and Programming, Version 4.10.0. 2018. URL: https://www.gap-system.org 

It is impossible to overstate the importance of Sylow's theorems in the study of finite groups. Without it the subject would not get off the ground.

— ISRAEl NATHAN HERSTEIN 



\title{
Prime power indices in factorised groups
}

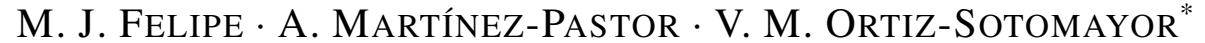

\begin{abstract}
Let the group $G=A B$ be the product of the subgroups $A$ and $B$. We determine some structural properties of $G$ when the $p$-elements in $A \cup B$ have prime power indices in $G$, for some prime $p$. More generally, we also consider the case that all prime power order elements in $A \cup B$ have prime power indices in $G$. In particular, when $G=A=B$ we obtain as a consequence some known results.
\end{abstract}

Keywords Finite groups · Products of groups · Conjugacy classes · Sylow subgroups

2010 MSC 20D10 $\cdot 20 \mathrm{D} 40 \cdot 20 \mathrm{E} 45 \cdot 20 \mathrm{D} 20$

\section{Introduction}

Along this paper all groups considered are finite. Throughout the last decades, the impact of conjugacy class sizes (also called indices) over the structure of finite groups has been highly investigated. Simultaneously, several authors have studied groups factorised as the product of two subgroups, in particular when those factors verify certain relations of permutability (see [4] for a detailed account on this subject). In this setting a main problem is how to infer structural properties from the factors to the whole group. The purpose of this paper is to present new achievements in the study of finite groups which combine both current research lines. Although the literature in this context is sparse, a first approach can be found either in [3], [10], or [12], where square-free class sizes were analysed. In this line, our concrete goal here is to obtain some structural facts about a factorised group, provided that the indices of certain prime power order elements in the factors are also prime powers.

One of the usual troubles in the framework of conjugacy classes is that, a priori, it is not guaranteed that the indices of the elements in a subgroup divide the corresponding indices in the whole group. Surprisingly, under our hypotheses, we have been able to prove that this

\footnotetext{
*The first author is supported by Proyecto Prometeo II/2015/011, Generalitat Valenciana (Spain), and the second author is supported by Proyecto MTM2014-54707-C3-1-P, Ministerio de Economía, Industria y Competitividad (Spain). The results in this paper are part of the third author's Ph.D. thesis, and he acknowledges the predoctoral grant ACIF/2016/170, Generalitat Valenciana (Spain).

Instituto Universitario de Matemática Pura y Aplicada (IUMPA-UPV), Universitat Politècnica de València, Camino de Vera s/n, 46022, Valencia, Spain.

凶: mfelipe@mat.upv.es, anamarti@mat.upv.es, vicorso@doctor.upv.es

ORCID iDs: 0000-0002-6699-3135, 0000-0002-0208-4098, 0000-0001-8649-5742
} 
happens for the considered elements in the factors of a factorised group (see Proposition $\mathrm{D}$ ). We also highlight that, in our development, we do not use any permutability property between the factors, in contrast to what occurs in the three above cited papers.

The origin of our research can be located in the manuscript of Baer [2], where the main result characterises all finite groups such that every prime power order element has prime power index. In 1990, Chillag and Herzog $([\overline{\mathbf{8}}])$ analysed groups all of whose conjugacy classes have prime power size. Later on, these studies were enhanced by Camina and Camina in [6]. Instead of imposing the prime power index condition on all (prime power order) elements, they restricted focus only to those elements with order a $p$-number for a fixed prime $p$. Next, in 2005, Berkovich and Kazarin $([\mathbf{5}])$ addressed also several problems about prime power indices in finite groups. In particular, in both papers [5] and [6], two alternative shorter proofs of the aforementioned Baer's characterisation are provided.

We will use the following terminology: for a group $G$ and an element $x \in G$, we call $i_{G}(x)$ the index of $x$ in $G$, that is, $i_{G}(x)=\left|G: \mathbf{C}_{G}(x)\right|$ is the size of the conjugacy class $x^{G}$. For a natural number $n$, we denote by $\pi(n)$ the set of prime divisors of $n$. In particular, $\pi(G)$ is the set of prime divisors of the order of $G$. If $p$ is a prime, then the set of all Sylow $p$-subgroups of $G$ is represented by $\operatorname{Syl}_{p}(G)$, and $\operatorname{Hall}_{\pi}(G)$ denotes the set of all Hall $\pi$-subgroups of $G$ for a set of primes $\pi$. A group such that $G=\mathbf{O}_{\pi}(G) \times \mathbf{O}_{\pi^{\prime}}(G)$ is said to be $\pi$-decomposable. Given a group $G=A B$ which is the product of the subgroups $A$ and $B$, a subgroup $S$ is called prefactorised (with respect to this factorisation) if $S=(S \cap A)(S \cap B)$ (see [1] $]$ ). The remaining notation and terminology is standard in this topic, and it is taken mainly from [9]. We also refer to this book for details about classes of groups.

According to the paper of Camina and Camina [6], given a group $G$ and a prime $p \in \pi(G)$, we call $G$ a $p$-Baer group if every $p$-element has prime power index (hereafter, the natural number 1 is a power of every prime). Moreover, if each prime power order element has prime power index, $G$ is called a Baer group. Inspired by those definitions, we introduce the following concepts for factorised groups:

Definition. Let $G=A B$ be the product of the subgroups $A$ and $B$, and let $p \in \pi(G)$. We say that:

- $G=A B$ is a $\boldsymbol{p}$-Baer factorisation if $i_{G}(x)$ is a prime power for every $p$-element $x \in A \cup B$;

- $G=A B$ is a Baer factorisation if $i_{G}(x)$ is a prime power for all prime power order elements $x \in A \cup B$, i.e., if it is a $p$-Baer factorisation for all $p$.

Clearly, any central product of two ( $p$-)Baer groups provides a ( $p$-)Baer factorisation.

Our first outcome is to determine structural information of a finite group $G$ which has a $p$-Baer factorisation:

Theorem A. Let $G=A B$ be a $p$-Baerfactorisation, and let $P \in \operatorname{Syl}_{p}(G)$. Then:

(1) $G / \mathbf{C}_{G}\left(\mathbf{O}_{p}(G)\right)$ is p-decomposable.

(2) Both $P \mathbf{F}(G)$ and $P \mathrm{O}_{p^{\prime}}(G)$ are normal in $G$. In particular, $G$ is p-soluble of p-length 1.

(3) The Sylow p-subgroup of $G / \mathbf{F}(G)$ is abelian.

(4) $P$ is abelian if and only if $\mathbf{O}_{p}(G)$ so is.

(5) If $P=(P \cap A)(P \cap B)$ and $P \cap X \nless \mathbf{C}_{G}\left(\mathbf{O}_{p}(G)\right)$ for some $X \in\{A, B\}$, then $P \cap X$ centralises every Hall $p^{\prime}$-subgroup of $G$.

(6) If the Sylow p-subgroups of $A$ and $B$ are non-abelian, then $G$ is p-decomposable.

Besides, we get additional information based on the primes appearing as indices of the $p$ elements in the factors of a $p$-Baer factorisation: 
Theorem B. Let $G=A B$ be a $p$-Baer factorisation, and let $P \in \operatorname{Syl}_{p}(G)$. Then there exist unique primes $q$ and $r$ such that $i_{G}(x)$ is a q-number for every p-element $x \in A$, and $i_{G}(y)$ is an $r$-number for every p-element $y \in B$, respectively. (Possibly $p \in\{q, r\}$ or $q=r$.)

Moreover, $P \leqslant \mathbf{C}_{G}\left(\mathbf{O}_{\{q, r\}^{\prime}}(\mathbf{F}(G))\right)$, and $P \mathbf{O}_{q}(G) \mathbf{O}_{r}(G)$ is normal in $G$. Further:

(1) If $q=r=p$, then $G$ is $p$-decomposable.

(2) If $p \notin\{q, r\}$, then $P$ is abelian.

In the particular case when $G=A=B$ in the above result and Theorem $\mathrm{A}$ (2), we partially recover [6. Theorem A] due to Camina and Camina (see Section 3, Corollary 3).

Afterwards, we impose the prime power index condition on all prime power order elements in the factors (that is, we consider groups with a Baer factorisation). We start proving the main theorem of Baer's paper [2] from our results on $p$-Baer factorisations when $G=A=B$ (see Theorem 1).

Then our first result for a non-trivial Baer factorisation is the next consequence of Theorem A

Corollary C. If $G=A B$ is a Baer factorisation, then:

(1) $G / \mathbf{F}(G)$ is abelian.

(2) $G$ has abelian Sylow subgroups (that is, $G$ is an A-group) if and only if $\mathbf{F}(G)$ is abelian.

(3) Set $\sigma:=\left\{p \in \pi(G) \mid A_{p} \in \operatorname{Syl}_{p}(A)\right.$ and $B_{p} \in \operatorname{Syl}_{p}(B)$ are non-abelian $\}$. Then $G=\mathbf{O}_{\sigma}(G) \times \mathbf{O}_{\sigma^{\prime}}(G)$ with $\mathbf{O}_{\sigma}(G)$ nilpotent.

(4) If all Sylow subgroups of $A$ and $B$ are non-abelian, then $G$ is nilpotent.

It is worthwhile to wonder whether the factors of a Baer factorisation are Baer groups. We have obtained that the answer is positive, in relation to the above comments on the divisibility of the indices:

Proposition D. Let $G=A B$ be a Baer factorisation. Let $x \in X$ be a prime power order element, where $X \in\{A, B\}$. If $i_{G}(x)$ is a q-number for some prime $q$, then $i_{X}(x)$ is also a $q$-number. In particular, it follows that $A$ and $B$ are Baer groups.

Consequently, the structure of $A$ and $B$ in a Baer factorisation $G=A B$ is well-known. Nevertheless, we cannot expect to get an analogous characterisation as Baer's one for Baer factorisations, even for direct products $G=A \times B$ (see Example 2 (i)).

At best, some arithmetical and structural information about Baer factorisations arises locally, i.e., prime by prime:

Theorem E. Let $G=A B$ be a Baer factorisation. For a prime $p$, and given $P \in \operatorname{Syl}_{p}(G)$ :

(1) If $P$ is not abelian, then $\left|G: \mathbf{C}_{G}(P)\right|$ is a $\{p, q\}$-number, for a prime $q .($ Possibly, $p=q$.)

(2) If $P$ is abelian, then $\left|G: \mathbf{C}_{G}(P)\right|$ is a $\{q, r\}$-number, for some primes $q$ and $r$, both distinct from $p$. (Possibly $q=r$.)

Further, $G / \mathbf{C}_{G}\left(\mathbf{O}_{p}(G)\right)$ is p-decomposable with abelian p-complement, and the p-complement has order divisible by at most two primes.

Finally, we have attained a characterisation of Baer factorisations through the indices of the centralisers of the Sylow subgroups of the factors:

Theorem F. Let $G=A B$ be the product of the subgroups $A$ and $B$. Then this is a Baer factorisation if and only if $\left|G: \mathbf{C}_{G}\left(A_{p}\right)\right|$ and $\left|G: \mathbf{C}_{G}\left(B_{p}\right)\right|$ are prime powers, for $A_{p} \in \operatorname{Syl}_{p}(A)$ and $B_{p} \in \operatorname{Syl}_{p}(B)$, and for every prime $p$.

In Section 3 we prove Theorems $\mathrm{A}$ and $\mathrm{B}$, which refer to prime power indices of $p$-elements, for a fixed prime $p$. The remaining stated results, which consider prime power order elements (for all primes), are proved in Section 4 . We illustrate the scope of our research with some examples. 


\section{Preliminary results}

We will use the following elementary properties frequently, sometimes without further reference.

Lemma 1. Let $N$ be a normal subgroup of a group $G$, and let $p$ be a prime. Then:

(a) $i_{N}(x)$ divides $i_{G}(x)$, for any $x \in N$.

(b) $i_{G / N}(x N)$ divides $i_{G}(x)$, for any $x \in G$.

(c) If $x N$ is a p-element of $G / N$, then there exists a p-element $x_{1} \in G$ such that $x N=x_{1} N$.

The next result about Sylow subgroups of factorised groups will be useful along the paper. It is a convenient reformulation of [1, 1.3.3].

Lemma 2. [1, 1.3.3] Let $G=A B$ be the product of the subgroups $A$ and $B$. Then for each $p \in \pi(G)$ there exists $P \in \operatorname{Syl}_{p}(G)$ such that $P=(P \cap A)(P \cap B)$, with $P \cap A \in \operatorname{Syl}_{p}(A)$ and $P \cap B \in \operatorname{Syl}_{p}(B)$.

Remark 1. We call attention to some facts on Sylow subgroups of factorised groups which will be used sometimes with no citation. Let $G=A B$ be the product of the subgroups $A$ and $B$, and let $p$ be a prime.

(1) Consider a Sylow $p$-subgroup $P=(P \cap A)(P \cap B)$ of $G$ such that $P \cap A \in \operatorname{Syl}_{p}(A)$ and $P \cap B \in \operatorname{Syl}_{p}(B)$. Then imposing arithmetical conditions on the indices of the $p$-elements in $A \cup B$ is equivalent to impose them on the indices of the elements in $(P \cap A) \cup(P \cap B)$, because of the conjugacy of Sylow $p$-subgroups.

(2) There exist easy examples which show that not every prefactorised Sylow $p$-subgroup $P=(P \cap A)(P \cap B)$ verifies that $P \cap A \in \operatorname{Syl}_{p}(A)$ and $P \cap B \in \operatorname{Syl}_{p}(B)$.

(3) In general, $\mathbf{O}_{p}(G)$ does not need to be prefactorised. However, if $P=(P \cap A)(P \cap B) \in$ $\operatorname{Syl}_{p}(G)$ with either $P \cap A \leqslant \mathbf{F}(G)$ or $P \cap B \leqslant \mathbf{F}(G)$, then by the Dedekind law we get that in this case $\mathbf{O}_{p}(G)$ is prefactorised.

The following result is due to Wielandt.

Lemma 3. [2. Lemma 6] Let $G$ be a finite group and $p \in \pi(G)$. If $x \in G$ is a p-element and $i_{G}(x)$ is a $p$-number, then $x \in \mathbf{O}_{p}(G)$.

In [6], Camina and Camina proved the next proposition, which extends the above lemma and a well-known result of Burnside about the non-simplicity of groups with a conjugacy class of prime power size.

Proposition 1. [6. Theorem 1] Let $G$ be a finite group. Then all elements of prime power index lie in $\mathbf{F}_{2}(G)$, the second term of the Fitting series of $G$.

Finally, the lemma below, which is due to Berkovich and Kazarin, is a key fact in the proof of Theorem B,

Lemma 4. [5, Lemma 4] Let $G$ be a finite group, and let $p$ be a prime. Suppose that the $p$ elements $x, y \in G \backslash \mathbf{Z}(G)$ are such that $i_{G}(x)$ and $i_{G}(y)$ are powers of distinct primes, and that $i_{G}(x y)$ is also a power of a prime. Then $\langle x, y\rangle^{G} \leqslant \mathbf{O}_{p}(G)$ and $i_{G}(x y)=\max \left\{i_{G}(x), i_{G}(y)\right\}$ is a power of $p$, so a Sylow p-subgroup of $G$ is non-abelian.

\section{Groups with a $p$-Baer factorisation}

In this section we will prove Theorems $\mathrm{A}$ and $\mathrm{B}$ via a series of results. Firstly, we show two facts about $p$-decomposability in $p$-Baer factorisations. 
Lemma 5. Let $G=A B$ be the product of the subgroups $A$ and $B$, and let $p$ be a prime. Then $i_{G}(x)$ is a p-number for each p-element $x \in A \cup B$ if and only if $G$ is p-decomposable.

Proof. Only the necessity of the condition is in doubt. Let $P=(P \cap A)(P \cap B) \in \operatorname{Syl}_{p}(G)$, which exists in virtue of Lemma 2 . The hypotheses and Lemma 3 lead to $x \in \mathbf{O}_{p}(G)$, for every $x \in(P \cap A) \cup(P \cap B)$. It follows that $P$ is normal in $G$ and so $G=\mathbf{O}_{p}(G) H$, with $H$ a Hall $p^{\prime}$-subgroup of $G$.

It remains only to prove that $\left[H, \mathbf{O}_{p}(G)\right]=1$. We may assume $\mathbf{O}_{p}(G) \neq 1$, so there exists a minimal normal subgroup $N$ of $G$ such that $N \leqslant \mathbf{O}_{p}(G)$. Since the class of $p$-decomposable groups is a saturated formation and the hypotheses hold for quotients of $G$, it follows by induction on $|G|$ that $N$ is the unique minimal normal subgroup of $G$ and $N=\mathbf{F}(G)=\mathbf{C}_{G}(N)=$ $\mathbf{O}_{p}(G)$ (see $\left[\mathbf{9}\right.$. A - 15.2, 15.8]). Consequently, since each element in $\left(\mathbf{O}_{p}(G) \cap A\right) \cup\left(\mathbf{O}_{p}(G) \cap B\right)$ has index a $p$-number and $\mathbf{O}_{p}(G)$ is abelian, it follows that all of them are central in $G$. This fact yields $\mathbf{O}_{p}(G)=\left(\mathbf{O}_{p}(G) \cap A\right)\left(\mathbf{O}_{p}(G) \cap B\right) \leqslant \mathbf{Z}(G)$ and the claim is proved.

Corollary 1. If $G=A B$ is a p-Baer factorisation, then $G / \mathbf{C}_{G}\left(\mathbf{O}_{p}(G)\right)$ is p-decomposable.

Proof. We can assume $1 \neq \mathbf{O}_{p}(G) \nless \mathbf{Z}(G)$. Denote $\bar{G}:=G / \mathbf{C}_{G}\left(\mathbf{O}_{p}(G)\right)$. If $\bar{G}$ is a $p^{\prime}$-group the result follows, so let $1 \neq \bar{x} \in \bar{A} \cup \bar{B}$ be a $p$-element. Then we can consider a $p$-element $x \in A \cup B$ such that $\bar{x}=x \mathbf{C}_{G}\left(\mathbf{O}_{p}(G)\right)$, and $i_{G}(x)$ is a prime power. But since $x \notin \mathbf{C}_{G}\left(\mathbf{O}_{p}(G)\right)$, it follows that $i_{G}(x)$ is a power of $p$, and $i_{\bar{G}}(\bar{x})$ so is. Finally, the previous lemma applies.

The lemma below provides the proof of Theorem $\mathrm{A}(2)$.

Lemma 6. Let $G=A B$ be a $p$-Baer factorisation, and let $P \in \operatorname{Syl}_{p}(G)$. Then:

(a) $P \mathbf{F}(G)$ is normal in $G$.

(b) $P \mathbf{O}_{p^{\prime}}(G)$ is normal in $G$. In particular, $G$ is p-soluble of p-length 1 .

Proof. (a) Let $P=(P \cap A)(P \cap B) \in \operatorname{Syl}_{p}(G)$, which exists by virtue of Lemma 2 . By Proposition 1 and our assumptions, we have $x \in \mathbf{F}_{2}(G)$ for every element $x \in(P \cap A) \cup(P \cap B)$. Therefore $P \leqslant \mathbf{F}_{2}(G)$, so $P \mathbf{F}(G) / \mathbf{F}(G)=\mathbf{O}_{p}(G / \mathbf{F}(G))$ and $P \mathbf{F}(G)$ is normal in $G$.

(b) We proceed by induction on $|G|$. If $N:=\mathbf{O}_{p^{\prime}}(G)=1$, then the result follows by (a). Hence we may assume $N \neq 1$. Since $\bar{G}:=G / N$ inherits the hypotheses by Lemma 1 , then $\bar{P} \mathbf{O}_{p^{\prime}}(\bar{G})=P N / N$ is normal in $G / N$, and the claim is proved.

Note that the existence of Hall $p^{\prime}$-subgroups in $p$-Baer factorisations is guaranteed as a consequence of the $p$-solubility of such groups. Indeed, if $G=A B$ is a Baer factorisation (i.e., it is $p$-Baer for all $p$ ), then it follows that $G / \mathbf{F}(G)$ is nilpotent. This fact will be strengthened later (see Proposition C).

Proposition 2. Let $G=A B$ be a p-Baer factorisation, and let $P=(P \cap A)(P \cap B) \in \operatorname{Syl}_{p}(G)$.

(a) If for some $X \in\{A, B\}$ it holds that $P \cap X \nless \mathbf{F}(G)$, then $P \cap X \leqslant \mathbf{C}_{G}\left(\mathbf{O}_{p}(G)\right), P \cap X$ is abelian, and $[P \cap A, P \cap B]=1$.

(b) If both $P \cap A \nless \mathbf{F}(G)$ and $P \cap B \nless \mathbf{F}(G)$, then $P$ is abelian.

(c) The Sylow p-subgroup of $G / \mathbf{F}(G)$ is abelian.

(d) $P$ is abelian if and only if $\mathbf{O}_{p}(G)$ so is.

(e) If $P \cap X \nless \mathbf{C}_{G}\left(\mathbf{O}_{p}(G)\right)$ for some $X \in\{A, B\}$, then $P \cap X \leqslant \mathbf{C}_{G}(H)$ for every $H \in \operatorname{Hall}_{p^{\prime}}(G)$. In particular, this holds when $P \cap X$ is non-abelian.

Proof. (a) Let $x \in(P \cap X) \backslash \mathbf{O}_{p}(G)$. Then $i_{G}(x)$ is a power of a prime $q \neq p$ by Lemma 3 , so $x \in \mathbf{C}_{G}\left(\mathbf{O}_{p}(G)\right)$. Now let $y \in \mathbf{O}_{p}(G) \cap X$. Then $x y \in(P \cap X) \backslash \mathbf{O}_{p}(G)$, so $x y \in \mathbf{C}_{G}\left(\mathbf{O}_{p}(G)\right)$ and $y \in \mathbf{C}_{G}\left(\mathbf{O}_{p}(G)\right)$. It follows $P \cap X \leqslant \mathbf{C}_{G}\left(\mathbf{O}_{p}(G)\right)$ and the first claim is proved.

Now we show that $P \cap X$ is abelian. By Lemma 6 (b), we get $\mathbf{O}_{p^{\prime}}(G) \neq 1$, and for all $g \in G$ it holds that $P^{g} \leqslant\left(P \mathbf{O}_{p^{\prime}}(G)\right)^{g}=P \mathbf{O}_{p^{\prime}}(G)$, so there exists $t_{g} \in \mathbf{O}_{p^{\prime}}(G)$ such that 
$P^{g}=P^{t_{g}}$. Let $x \in(P \cap X) \backslash \mathbf{O}_{p}(G)$. By the assumptions and Lemma 3 , we have that $i_{G}(x)$ is a power of a prime $r \neq p$, so there exists $P^{g}=P^{t_{g}} \leqslant \mathbf{C}_{G}(x)$. Hence $x \in \mathbf{C}_{G}(P) \mathbf{O}_{p^{\prime}}(G) \leqslant$ $\mathbf{C}_{G}(P \cap X) \mathbf{O}_{p^{\prime}}(G)$. On the other hand, if $x \in \mathbf{O}_{p}(G) \cap X$, then $x \in \mathbf{C}_{G}(P \cap X)$ by the first paragraph. It follows $P \cap X \leqslant \mathbf{C}_{G}(P \cap X) \mathbf{O}_{p^{\prime}}(G)$, and $[P \cap X, P \cap X] \leqslant P \cap[P \cap$ $\left.X, \mathbf{O}_{p^{\prime}}(G)\right]\left[P \cap X, \mathbf{C}_{G}(P \cap X)\right] \leqslant P \cap \mathbf{O}_{p^{\prime}}(G)=1$.

Finally, we prove that $[P \cap A, P \cap B]=1$. Take for instance $X=A$. If $P \cap B \leqslant \mathbf{F}(G)$, then the claim is clear since $P \cap A \leqslant \mathbf{C}_{G}\left(\mathbf{O}_{p}(G)\right)$. We may assume $P \cap B \nless \mathbf{F}(G)$, and so $\mathbf{O}_{p}(G) \leqslant \mathbf{C}_{G}(P \cap B)$. Moreover, as in the previous paragraph, if $x \in(P \cap A) \backslash \mathbf{O}_{p}(G)$, then $x \in \mathbf{C}_{G}(P \cap B) \mathbf{O}_{p^{\prime}}(G)$. Hence $P \cap A \leqslant \mathbf{C}_{G}(P \cap B) \mathbf{O}_{p^{\prime}}(G)$, and $[P \cap A, P \cap B]=1$.

(b) It is a direct consequence of (a).

(c) The uniqueness of the Sylow $p$-subgroup of $G / \mathbf{F}(G)$ follows from Lemma 6 (a). If both $P \cap A \nless \mathbf{F}(G)$ and $P \cap B \nless \mathbf{F}(G)$, then $P$ is abelian by (b), and so is $P \mathbf{F}(G) / \mathbf{F}(G)$. Finally, if for instance $P \cap A \nless \mathbf{F}(G)$ and $P \cap B \leqslant \mathbf{F}(G)$, then $P \cap A$ is abelian by (a) and $P \mathbf{F}(G) / \mathbf{F}(G)=(P \cap A) \mathbf{F}(G) / \mathbf{F}(G)$ is also abelian.

(d) Let us show that if $P$ is not abelian, then $\mathbf{O}_{p}(G)$ cannot be abelian. Applying (b), we can assume for instance that $P \cap A \leqslant \mathbf{F}(G)$ and $P \cap B \nless \mathbf{F}(G)$. Then by (a) we have that $P \cap B$ is abelian and $[P \cap A, P \cap B]=1$. Therefore $P \cap A=\mathbf{O}_{p}(G) \cap A$ cannot be abelian, and so $\mathbf{O}_{p}(G)$ is not abelian either.

(e) By (a) it holds that $P \cap X=\mathbf{O}_{p}(G) \cap X$. Let $x \in(P \cap X) \backslash \mathbf{C}_{G}\left(\mathbf{O}_{p}(G)\right)$. Then $\mathbf{O}_{p}(G) \nless \mathbf{C}_{G}(x)$, and $i_{G}(x)$ is a power of $p$. Since $x \in \mathbf{O}_{p}(G)$, then $\mathbf{C}_{G}\left(\mathbf{O}_{p}(G)\right) \leqslant \mathbf{C}_{G}(x)$, so $\left|G / \mathbf{C}_{G}\left(\mathbf{O}_{p}(G)\right): \mathbf{C}_{G}(x) / \mathbf{C}_{G}\left(\mathbf{O}_{p}(G)\right)\right|=\left|G: \mathbf{C}_{G}(x)\right|$, which is a power of $p$. By Corollary 1. $G / \mathbf{C}_{G}\left(\mathbf{O}_{p}(G)\right)$ is $p$-decomposable, and its unique Hall $p^{\prime}$-subgroup is contained in $\mathbf{C}_{G}(x) / \mathbf{C}_{G}\left(\mathbf{O}_{p}(G)\right)$. Thus if $H \in \operatorname{Hall}_{p^{\prime}}(G)$, we deduce $H^{g} \leqslant \mathbf{C}_{G}(x)$ for every $g \in G$, and for all $x \in(P \cap X) \backslash \mathbf{C}_{G}\left(\mathbf{O}_{p}(G)\right)$. On the other hand, given $y \in P \cap X \cap \mathbf{C}_{G}\left(\mathbf{O}_{p}(G)\right)$, if $x \in(P \cap X) \backslash \mathbf{C}_{G}\left(\mathbf{O}_{p}(G)\right)$, then $x y \in(P \cap X) \backslash \mathbf{C}_{G}\left(\mathbf{O}_{p}(G)\right)$, so $H^{g} \leqslant \mathbf{C}_{G}(x y) \cap \mathbf{C}_{G}(x)=$ $\mathbf{C}_{G}(x) \cap \mathbf{C}_{G}(y) \leqslant \mathbf{C}_{G}(y)$. Therefore $H^{g} \leqslant \mathbf{C}_{G}(P \cap X)$ for all $g \in G$, and the first claim follows.

Finally, if $P \cap X$ is non-abelian, then by (a) $P \cap X \leqslant \mathbf{O}_{p}(G)$ and so $P \cap X \nless \mathbf{C}_{G}\left(\mathbf{O}_{p}(G)\right)$.

Corollary 2. Let $G=A B$ be a p-Baer factorisation. If the Sylow p-subgroups of $A$ and $B$ are non-abelian, then $G$ is p-decomposable.

Proof. It is sufficient to take $P=(P \cap A)(P \cap B) \in \operatorname{Syl}_{p}(G)$ with $P \cap A \in \operatorname{Syl}_{p}(A)$ and $P \cap B \in \operatorname{Syl}_{p}(B)$ and to apply the last statement of the above proposition.

If we combine the previous results, we get the proof of Theorem A.

Proof of Theorem A. The statement (1) is exactly Corollary 1. Lemma 6 yields (2), whilst claims (3), (4) and (5) are Proposition 2 (c), (d) and (e), respectively. Corollary 2 gives the last assertion.

In the remainder of the section, we focus on proving Theorem $B$,

Lemma 7. Let $G=A B$ be a p-Baer factorisation. Let $P=(P \cap A)(P \cap B) \in \operatorname{Syl}_{p}(G)$. If for some $X \in\{A, B\}$ it holds $P \nless \mathbf{C}_{G}\left(\mathbf{O}_{p}(G) \cap X\right)$, then $\left|G: \mathbf{C}_{G}(P \cap X)\right|$ is a p-number.

Proof. If $P \cap X \nless \mathbf{F}(G)$, then by Proposition 2 (a) we get a contradiction. Therefore $P \cap$ $X=\mathbf{O}_{p}(G) \cap X$. Since $P \nless \mathbf{C}_{G}\left(\mathbf{O}_{p}(G) \cap X\right)$, we have that either $P \cap X$ is non-abelian or $[P \cap X, P \cap Y] \neq 1$ where $\{A, B\}=\{X, Y\}$. In the last case, $P \cap Y \leqslant \mathbf{F}(G)$ by Proposition 2 (a), and therefore $P \cap X \nless \mathbf{C}_{G}\left(\mathbf{O}_{p}(G)\right)$. Thus we can apply in both cases Proposition 2 (e) to deduce $P \cap X \leqslant \mathbf{C}_{G}(H)$, for every $H \in \operatorname{Hall}_{p^{\prime}}(G)$. It follows that $\left|G: \mathbf{C}_{G}(P \cap X)\right|$ is a power of $p$. 
Proposition 3. Let $G=A B$ be a $p$-Baer factorisation, and let $P \in \operatorname{Syl}_{p}(G)$. Then:

(a) There exist unique primes $q$ and $r$ such that $i_{G}(x)$ is a q-number for every p-element $x \in A$, and $i_{G}(y)$ is an $r$-number for every p-element $y \in B$, respectively. (Possibly $p \in\{q, r\}$ or $q=r$.)

(b) $P \leqslant \mathbf{C}_{G}\left(\mathbf{O}_{\{q, r\}^{\prime}}(\mathbf{F}(G))\right)$ and $P \mathbf{O}_{q}(G) \mathbf{O}_{r}(G)$ is normal in $G$.

(c) If $p \notin\{q, r\}$, then $P$ is abelian.

Proof. By Lemma 2 we can assume that $P=(P \cap A)(P \cap B)$ where $P \cap A \in \operatorname{Syl}_{p}(A)$ and $P \cap B \in \operatorname{Syl}_{p}(B)$ :

(a) We argue for instance with $A$. If the result is false, then there exist $p$-elements $a_{1}, a_{2} \in A$ such that $1 \neq i_{G}\left(a_{1}\right)$ and $1 \neq i_{G}\left(a_{2}\right)$ are relatively prime. By the conjugacy of the Sylow $p$ subgroups in $A$, we may assume $a_{1}, a_{2} \in P \cap A$, and $i_{G}\left(a_{1} a_{2}\right)$ is a prime power. By Lemma 4. $i_{G}\left(a_{1} a_{2}\right)=\max \left\{i_{G}\left(a_{1}\right), i_{G}\left(a_{2}\right)\right\}$ is a $p$-number, and $a_{1}, a_{2} \in \mathbf{O}_{p}(G) \cap A$. Let assume that $\max \left\{i_{G}\left(a_{1}\right), i_{G}\left(a_{2}\right)\right\}=i_{G}\left(a_{1}\right)$. Since $a_{1}$ is not central, we get $P \notin \mathbf{C}_{G}\left(\mathbf{O}_{p}(G) \cap A\right)$, and Lemma 7 leads to $a_{2} \in \mathbf{Z}(G)$, the final contradiction.

(b) By (a) $\mathbf{O}_{\{q, r\}^{\prime}}(\mathbf{F}(G)) \leqslant \mathbf{C}_{G}(P \cap A) \cap \mathbf{C}_{G}(P \cap B)=\mathbf{C}_{G}(P)$. Applying Lemma 6 (a), we deduce that $P \mathbf{O}_{q}(G) \mathbf{O}_{r}(G)$ is normal in $G$.

(c) Let suppose that $P$ is not abelian. Then by Proposition 2 (d), $P \cap X \nless \mathbf{C}_{G}\left(\mathbf{O}_{p}(G)\right)$ for some $X \in\{A, B\}$. Finally, we deduce from Proposition 2 (e) that $\left|G: \mathbf{C}_{G}(P \cap X)\right|$ is a $p$-number, and so $p \in\{q, r\}$.

Example 1. The primes $q$ and $r$ in the previous result may not be equal. Let $G=A \times B$ be the direct product of a symmetric groups $A=\Sigma_{3}$ of three letters and a dihedral group $B=D_{10}$ of order ten, and consider the prime $p=2$. Clearly, that factorisation is 2-Baer. Nevertheless, the 2-elements $x \in A$ have $i_{G}(x)=3$ and the 2-elements $y \in B$ have $i_{G}(y)=5$. Moreover, if $P \in \operatorname{Syl}_{2}(G)$, neither $P \mathbf{O}_{3}(G)$ nor $P \mathbf{O}_{5}(G)$ are normal in $G$.

Finally, we are ready to prove Theorem $B$.

Proof of Theorem B. The existence of the unique primes $q$ and $r$ follows from Proposition 3 (a). Then, the statement (1) is Lemma 5, and the remaining assertions follow by Proposition 3 (b) and (c).

If we take the trivial factorisation $G=A=B$ in Theorems $\mathrm{A}(2)$ and $\mathrm{B}$, we partially recover the main theorem of Camina and Camina in $[\mathbf{6}]$ about $p$-Baer groups.

Corollary 3. [6. Theorem A] Let G be a p-Baer group for some prime $p$. Then:

(a) $G$ is $p$-soluble with $p$-length 1 , and

(b) there is a unique prime $q$ such that each p-element has q-power index.

Further, let $Q \in \operatorname{Syl}_{q}(G)$, then

(c) if $p=q, P$ is a direct factor of $G$, or

(d) if $p \neq q, P$ is abelian, and $P \mathbf{O}_{q}(G)$ is normal in $G$.

Finally, we emphasise the relation between the primes appearing as indices of the $p$-elements in the factors of a $p$-Baer factorisation.

Lemma 8. Let $G=A B$ be a $p$-Baer factorisation. Let assume that there exist non-central p-elements $a \in A$ and $b \in B$, so that $i_{G}(a)$ is a q-number and $i_{G}(b)$ is an $r$-number, for some primes $q$ and $r$.

Let assume that $q \neq p$ and the factorisation is also $q$-Baer. Then:

(a) If the q-elements in $A \cup B$ have indices an s-power, then $s \in\{p, r\}$.

(b) Moreover, if $q=r$ and $s$ is the prime in (a), then $s=p$ and a Hall $\{p, q\}$-subgroup of $G$ is normal with abelian Sylow subgroups. 
Proof. (a) Take $P=(P \cap A)(P \cap B) \in \operatorname{Syl}_{p}(G)$ such that $P \cap A \in \operatorname{Syl}_{p}(A)$ and $P \cap B \in$ $\operatorname{Syl}_{p}(B)$. We may assume that $a \in P \cap A$ and $b \in P \cap B$. Suppose that $s \neq p$, and we claim that $s=r$. If $s=q$, then by Lemma 5 we obtain that $G$ is $q$-decomposable, which contradicts that $1 \neq i_{G}(a)$ is a $q$-number and $a$ is a $q^{\prime}$-element. Hence, $s \notin\{p, q\}$. Now if we assume also that $s \neq r$, then $\pi\left(i_{G}(z)\right) \cap\{p, q, r\}=\emptyset$ for any $q$-element $z \in A \cup B$. Since $P \mathbf{O}_{q}(G) \mathbf{O}_{r}(G)$ is a normal $\{p, q, r\}$-subgroup of $G$ by Proposition $3\left(\right.$ b), given $Q=(Q \cap A)(Q \cap B) \in \operatorname{Syl}_{q}(G)$ it follows $P \cap A \leqslant P \mathbf{O}_{q}(G) \mathbf{O}_{r}(G) \leqslant \mathbf{C}_{G}(Q \cap A) \cap \mathbf{C}_{G}(Q \cap B)=\mathbf{C}_{G}(Q)$. But this contradicts again that $i_{G}(a) \neq 1$ is a $q$-number.

(b) By (a), we deduce $s \in\{p, r\}$. As above, since $q=r$ we get $s=p$ because of Lemma 5. As a consequence, Proposition 3 (b) yields that $P \mathbf{O}_{q}(G)$ and $Q \mathbf{O}_{p}(G)$ are normal in $G$, for $P \in \operatorname{Syl}_{p}(G)$ and $Q \in \operatorname{Syl}_{q}(G)$. Hence $P Q \mathbf{O}_{q}(G) \mathbf{O}_{p}(G)=P Q \unlhd G$, and it is a Hall $\{p, q\}$-subgroup of $G$. Finally, the Sylow subgroups of $P Q$ are abelian because of Proposition $3(\mathrm{c})$.

If we choose the trivial factorisation $G=A=B$ in the above result, we recover:

Corollary 4. [6. Lemma 5] Let $G$ be a $p$-Baer group and a $q$-Baer group for primes $p \neq q$. Suppose that all p-elements have q-power index. Then all q-elements have p-power index.

\section{Groups with a Baer factorisation}

In the sequel, the prime power index condition is imposed on all prime power order elements in the factors, that is, we consider Baer factorisations.

We start this section by proving Baer's theorem $([\overline{2}])$ as a consequence of the results obtained in Section 3, when we consider the trivial factorisation $G=A=B$.

Theorem 1. [2, Section 3 - Theorem] Let $G$ be a finite group. Each element $x \in G$ of prime power order has prime power index if and only if

$$
G=G_{1} \times G_{2} \times \cdots \times G_{r},
$$

where $G_{i}$ and $G_{j}$ have relatively prime orders for $i \neq j$, and if $G_{i}$ is not of prime order, then $\left|\pi\left(G_{i}\right)\right|=2$ and its Sylow subgroups are abelian.

Proof. The converse is clear. Let $P \in \operatorname{Syl}_{p}(G)$. If $P$ is not abelian, then $P$ is a direct factor of $G$ by Corollary 2. Therefore, all non-abelian Sylow subgroups of $G$ are direct factors of it.

Now suppose that $P$ is abelian and non-central in $G$. Hence there is a $p$-element $x \in G$ such that $1 \neq i_{G}(x)$ is a $q$-number, for some prime $q \neq p$. Necessarily, by Lemma 5, there is a $q$-element $y \in G$ such that $1 \neq i_{G}(y)$ is a $q^{\prime}$-number. For some $Q \in \operatorname{Syl}_{q}(G)$, Lemma 8 (b) yields that $P Q$ is a normal Hall $\{p, q\}$-subgroup of $G$, and $Q$ is also abelian.

Take a prime $r \notin\{p, q\}$ and $R \in \operatorname{Syl}_{r}(G)$. We may assume that $R$ is abelian and noncentral. Thus, for each element $z \in R$, we deduce $\pi\left(i_{G}(z)\right) \cap\{p, q\}=\emptyset$ by virtue of Lemma 8 (b) again. Consequently $P Q \leqslant \mathbf{C}_{G}(R)$. Since this is valid for all primes $r \notin\{p, q\}$, the $\{p, q\}$-decomposability of $G$ follows. The result is now established.

Remark 2. The results stated in Section 3 can be also used to give an alternative proof of $[\boldsymbol{8}$. Theorem 2] due to Chillag and Herzog, avoiding Theorem 1.

Proof of Corollary C. We deduce the first two statements from a direct application of Theorem $\mathrm{A}$ (3) and (4), respectively. The final two assertions follow from Theorem $\mathrm{A}(6)$.

Next we are proving that the factors of a Baer factorisation are Baer groups. This is because in such a factorisation $G=A B$, the prime power index condition is inherited by both factors, 
even if $A$ and $B$ are not subnormal in $G$. This is no longer true for other arithmetical conditions on the indices (see for instance [10] for the square-free property). In particular, as pointed out in [5], subgroups of Baer groups are also Baer groups. It is an open question whether the factors of a $p$-Baer factorisation are $p$-Baer groups. Nevertheless, it might happen for such a group that the indices in a factor and in the whole group are powers of distinct primes (see Final examples (2)).

Proof of Proposition D. Let $P=(P \cap A)(P \cap B) \in \operatorname{Syl}_{p}(G)$ such that $P \cap A \in \operatorname{Syl}_{p}(A)$ and $P \cap B \in \operatorname{Syl}_{p}(B)$, for some prime $p$. Let $X \in\{A, B\}$ and take $x \in(P \cap X) \backslash \mathbf{Z}(G)$ such that $i_{G}(x)$ is a $q$-number. If $q=p$, then Proposition 3 (a) and Lemma 3 yields $P \cap X=$ $\mathbf{O}_{p}(G) \cap X$. Moreover, $P \notin \mathbf{C}_{G}\left(\mathbf{O}_{p}(G) \cap X\right)$ because $1 \neq i_{G}(x)$ is a $p$-power. Thus, by virtue of Lemma 7, we deduce that $\left|G: \mathbf{C}_{G}(P \cap X)\right|$ is a $p$-power. As $P \cap X$ is normal in $X$, then $\left|X: \mathbf{C}_{X}(P \cap X)\right|=\left|X \mathbf{C}_{G}(P \cap X): \mathbf{C}_{G}(P \cap X)\right|$ divides $\left|G: \mathbf{C}_{G}(P \cap X)\right|$. Therefore $i_{X}(x)$ divides the $p$-number $\left|G: \mathbf{C}_{G}(P \cap X)\right|$.

Hence we may assume $q \neq p$. Suppose $\mathbf{O}_{q}(G) \neq 1$. Note that the quotient $\bar{G}:=$ $G / \mathbf{O}_{q}(G)$ inherits the hypotheses. It follows by induction that $i_{\bar{X}}(\bar{x})=\left|\bar{X}: \mathbf{C}_{\bar{X}}(\bar{x})\right|$ is a $q$-number, because $i_{\bar{G}}(\bar{x})$ divides $i_{G}(x)$. However, since $q \neq p$, applying [11, 3.2.8] and the isomorphism $\bar{X} \cong X /\left(X \cap \mathbf{O}_{q}(G)\right)$ we deduce $\mathbf{C}_{\bar{X}}(\bar{x})=\overline{\mathbf{C}_{X}(x)}$. Thus $\left|\bar{X}: \mathbf{C}_{\bar{X}}(\bar{x})\right|$. $\left|\left(X \cap \mathbf{O}_{q}(G)\right):\left(\mathbf{C}_{X}(x) \cap \mathbf{O}_{q}(G)\right)\right|=\left|X: \mathbf{C}_{X}(x)\right|$, which is also a $q$-power, and the result is proved in this case.

Now we assume $\mathbf{F}(G)=\mathbf{O}_{q^{\prime}}(\mathbf{F}(G))$. Let $M:=X \mathbf{F}(G)$ which is normal in $G$ by Corollary $\mathrm{C}(1)$. Then $M=X(M \cap Y)$ with $\{X, Y\}=\{A, B\}$, and $M$ verifies the hypotheses. If $M<$ $G$, by induction we get that $i_{X}(x)$ is a power of the same prime that divides $i_{M}(x)$, which divides $i_{G}(x)$. Consequently we may assume $G=M=X \mathbf{O}_{q^{\prime}}(\mathbf{F}(G))$. Let $G_{q^{\prime}} \in \operatorname{Hall}_{q^{\prime}}(G)$. Then $G_{q^{\prime}}=\mathbf{O}_{q^{\prime}}(\mathbf{F}(G))\left(X \cap G_{q^{\prime}}\right)$. Moreover, $\left|G: G_{q^{\prime}}\right|=\left|X \mathbf{O}_{q^{\prime}}(\mathbf{F}(G)):\left(X \cap G_{q^{\prime}}\right) \mathbf{O}_{q^{\prime}}(\mathbf{F}(G))\right|=$ $\left|X: X \cap G_{q^{\prime}}\right|$. Therefore, for each $G_{q^{\prime}} \in \operatorname{Hall}_{q^{\prime}}(G)$, we have that $X \cap G_{q^{\prime}}$ is also a Hall $q^{\prime}$-subgroup of $X$. Since $i_{G}(x)$ is a $q$-number, there exists some Hall $q^{\prime}$-subgroup of $G$ that centralises $x$, and so there exists a Hall $q^{\prime}$-subgroup of $X$ that centralises $x$, and we are done.

Example 2. (i) In contrast to Baer's theorem (Theorem 1), and in spite of the above proposition, in a Baer factorisation $G=A B$ it is not guaranteed that $G$ is a direct product of proper Hall subgroups for pairwise disjoint sets of primes, even for direct products: To see this consider $A=C_{3} \times\left[C_{7}\right] C_{2} \times\left[C_{11}\right] C_{5}$ and $B=C_{5} \times\left[C_{7}\right] C_{3} \times\left[C_{11}\right] C_{2}$. Then $G=A \times B$ is a Baer factorisation, but there are no pairwise coprime proper direct factors of $G$.

(ii) We highlight that there are Baer factorisations which are not just a central product of Baer groups: Let $G=H \times K$ be the direct product of a symmetric group $H=\Sigma_{3}$ and a dihedral group $K=D_{10}$. Let $A$ be a Sylow 2-subgroup of $K$, and let $B$ be the direct product of $H$ and the Sylow 5-subgroup of $K$. Then $G=A B$ is a Baer factorisation. Note that there is a 2-element $g \in G \backslash(A \cup B)$ such that $i_{G}(g)=15$, so $G$ is not a Baer group.

Now, as a step to prove Theorem E , an application of Lemma 5 gives the next result.

Corollary 5. Let $G=A B$ be the product of the subgroups $A$ and $B$, and let $p$ be a prime. Then $i_{G}(x)$ is a p-number for each prime power order element $x \in A \cup B$ if and only if $G=$ $\mathbf{O}_{p}(G) \times \mathbf{O}_{p^{\prime}}(G)$, and $\mathbf{O}_{p^{\prime}}(G)$ is abelian.

Proof. The sufficiency of the condition is straightforward. The $p$-decomposability of $G$ follows directly from Lemma 5. Finally, if we take a prime $q \neq p$ and a prefactorised Sylow $q$-subgroup $Q=(Q \cap A)(Q \cap B)$, then $i_{\mathbf{O}_{p^{\prime}}(G)}(x)=1$ for each element $x \in(Q \cap A) \cup(Q \cap B)$. Therefore $Q \leqslant \mathbf{Z}(G)$ for every $q \neq p$, and the result follows.

Furthermore, from the previous corollary we get: 
Corollary 6. Let $G=A B$ be the Baer factorisation. Then for each prime $p$ we have that $G / \mathbf{C}_{G}\left(\mathbf{O}_{p}(G)\right)$ is p-decomposable with abelian p-complement.

In the remainder of the section, we focus on proving Theorem $\mathrm{E}$,

Proposition 4. Let $G=A B$ be a Baer factorisation, and let $P \in \operatorname{Syl}_{p}(G)$. If $P \leqslant \mathbf{C}_{G}\left(\mathbf{O}_{p}(G) \cap\right.$ $X)$ for some $X \in\{A, B\}$, then $\mathbf{C}_{G}\left(\mathbf{O}_{p}(G) \cap X\right)$ is normal in $G$ and $G / \mathbf{C}_{G}\left(\mathbf{O}_{p}(G) \cap X\right)$ is an abelian q-group, for a prime $q \neq p$.

Proof. We denote $\widetilde{G}:=G / \mathbf{C}_{G}\left(\mathbf{O}_{p}(G)\right)$ and we have $\widetilde{G}=\mathbf{O}_{p}(\widetilde{G}) \times \widetilde{H}$ by Corollary 6 , where $\widetilde{H} \in \operatorname{Hall}_{p^{\prime}}(\widetilde{G})$ is abelian for any $H \in \operatorname{Hall}_{p^{\prime}}(G)$. Since $P \mathbf{C}_{G}\left(\mathbf{O}_{p}(G)\right) \leqslant \mathbf{C}_{G}\left(\mathbf{O}_{p}(G) \cap X\right)$, we get by the Dedekind law

$$
\mathbf{C}_{G}\left(\mathbf{O}_{p}(G) \cap X\right) / \mathbf{C}_{G}\left(\mathbf{O}_{p}(G)\right)=\mathbf{O}_{p}(\widetilde{G}) \times \widetilde{H_{0}} \unlhd \mathbf{O}_{p}(\widetilde{G}) \times \widetilde{H}=\widetilde{G},
$$

where $H_{0}:=H \cap \mathbf{C}_{G}\left(\mathbf{O}_{p}(G) \cap X\right)$, and thus $\mathbf{C}_{G}\left(\mathbf{O}_{p}(G) \cap X\right)$ is normal in $G$.

Set $\bar{G}:=G / \mathbf{C}_{G}\left(\mathbf{O}_{p}(G) \cap X\right)$. Then $\bar{G}$ is a $p^{\prime}$-group, and since it is isomorphic to a quotient of $\widetilde{G}$, it is abelian. We may affirm that there exists an element $x \in\left(\mathbf{O}_{p}(G) \cap X\right) \backslash \mathbf{Z}(G)$. Then $i_{G}(x)$ is a $q$-number for some prime $q \neq p$ (actually, this holds for every element in $\mathbf{O}_{p}(G) \cap X$ ). Moreover, $\left|\bar{G}: \overline{\mathbf{C}_{G}(x)}\right|=\left|G: \mathbf{C}_{G}(x)\right|$ so $q$ divides $|\bar{G}|$. Let suppose that there exists another prime $r \neq q$ such that $r$ divides $|\bar{G}|$. Since $\left|\overline{G:} \overline{\mathbf{C}_{G}(x)}\right|=\left|G: \mathbf{C}_{G}(x)\right|$, it follows that the unique Sylow $r$-subgroup $\bar{R}$ of $\bar{G}$ is contained in $\overline{\mathbf{C}_{G}(x)}$. Hence $R \leqslant \mathbf{C}_{G}(x)$ for every $x \in \mathbf{O}_{p}(G) \cap X$, so $R \leqslant \mathbf{C}_{G}\left(\mathbf{O}_{p}(G) \cap X\right)$, which contradicts that $r$ divides $|\bar{G}|$.

This last proposition is not longer true for $p$-Baer factorisations, as Final examples (1) shows. The next result is the last step to prove Theorem E.

Proposition 5. Let $G=A B$ be a Baer factorisation, and let $P=(P \cap A)(P \cap B) \in \operatorname{Syl}_{p}(G)$. Let assume that $P \cap X \nless \mathbf{F}(G)$ for some $X \in\{A, B\}$. Then:

(a) $P \cap X \leqslant \mathbf{Z}(P)$.

(b) There exists a unique prime $q \neq p$ such that $P \cap X \nless \mathbf{C}_{G}\left(\mathbf{O}_{q}(G)\right)$.

(c) $\left|G: \mathbf{C}_{G}(P \cap X)\right|$ is a power of the prime $q$ in statement $(b)$.

If, moreover, $P \cap Y \nless \mathbf{F}(G)$ where $\{X, Y\}=\{A, B\}$, then:

(d) $P$ is abelian and $\left|G: \mathbf{C}_{G}(P)\right|$ is a $\{q, r\}$-number, with $p \notin\{q, r\}, q$ is the prime in (b), and $r$ is the unique prime such that $P \cap Y \nless \mathbf{C}_{G}\left(\mathbf{O}_{r}(G)\right)$. (Possibly $q=r$.)

Proof. (a) This is exactly Proposition 2 (a).

(b) By (a), for every $x \in P \cap X$ it hold that $i_{G}(x)$ is a $q$-power, for a fixed prime $q \neq p$. Then $\mathbf{O}_{q^{\prime}}(\mathbf{F}(G)) \leqslant \mathbf{C}_{G}(P \cap X)$. Finally, $\mathbf{O}_{q}(G) \nless \mathbf{C}_{G}(P \cap X)$ since otherwise $P \cap X \leqslant$ $\mathbf{C}_{G}(\mathbf{F}(G)) \leqslant \mathbf{F}(G)$, a contradiction.

(c) Take $T \in \operatorname{Hall}_{\{p, q\}^{\prime}}(G)$ such that $P T$ is a $q$-complement of $G$. As $G / \mathbf{F}(G)$ is abelian by Proposition $\mathrm{C}$ (a), then $L:=P \mathbf{O}_{q}(G) T$ is normal in $G$. Consequently, for every $x \in P \cap X$ we obtain that $i_{L}(x)$ is a $q$-power, and there exists $g \in L$ such that $T^{g}$ centralises $x$, (actually by (a) we may assume $\left.g \in \mathbf{O}_{q}(G)\right)$. Set $K:=(P \cap X) \mathbf{O}_{q}(G)$. Hence $K \subseteq \cup_{g \in K}\left(\mathbf{C}_{K}(T) \mathbf{O}_{q}(G)\right)^{g} \subseteq$ $K$. It follows that $K=\mathbf{C}_{K}(T) \mathbf{O}_{q}(G)$ and $[P \cap X, T] \leqslant[K, T]=\left[\mathbf{C}_{K}(T) \mathbf{O}_{q}(G), T\right]=$ $\left[\mathbf{O}_{q}(G), T\right] \leqslant \mathbf{O}_{q}(G)$. But $[P \cap X, T] \leqslant P T$, which is a $q^{\prime}$-group. Then $[P \cap X, T]=1$, and $P$ also centralises $P \cap X$ by (a). The claim is now proved.

(d) Certainly $P$ is abelian by (a). Moreover, by (c), $\left|G: \mathbf{C}_{G}(P \cap A)\right|$ is a $q$-number and $\left|G: \mathbf{C}_{G}(P \cap B)\right|$ is an $r$-number for some primes $q$ and $r$. Note that $\mathbf{C}_{G}(P)=\mathbf{C}_{G}(P \cap$ $A) \cap \mathbf{C}_{G}(P \cap B)$. If $q \neq r$, then $G=\mathbf{C}_{G}(P \cap A) \mathbf{C}_{G}(P \cap B)$, and thus $\left|G: \mathbf{C}_{G}(P)\right|=$ $\left|G: \mathbf{C}_{G}(P \cap A)\right| \cdot\left|G: \mathbf{C}_{G}(P \cap B)\right|$ is a $\{q, r\}$-number. Therefore we may assume that $q=r$, and so every $p$-element in $A \cup B$ has $q$-power index. Arguing analogously as in (c), for a 
$T \in \operatorname{Hall}_{\{p, q\}^{\prime}}(G)$ such that $P T$ is a $q$-complement, we deduce that $P \mathbf{O}_{q}(G)=(P \cap A)(P \cap$ $B) \mathbf{O}_{q}(G) \leqslant \mathbf{C}_{G}(T) \mathbf{O}_{q}(G)$, and $[P, T] \leqslant P T \cap \mathbf{O}_{q}(G)=1$. Then $T$ centralises $P$, and $P$ is abelian, so $\left|G: \mathbf{C}_{G}(P)\right|$ is a $q$-power. The result is now established.

Proof of Theorem E. (1) Let $P=(P \cap A)(P \cap B) \in \operatorname{Syl}_{p}(G)$ non-abelian. If $P=\mathbf{O}_{p}(G)$, then either $P \notin \mathbf{C}_{G}\left(\mathbf{O}_{p}(G) \cap A\right)$ or $P \notin \mathbf{C}_{G}\left(\mathbf{O}_{p}(G) \cap B\right)$. Assume for instance that $P \notin$ $\mathbf{C}_{G}\left(\mathbf{O}_{p}(G) \cap A\right)$. Then by Lemma 7, we get that $\left|G: \mathbf{C}_{G}(P \cap A)\right|$ is a $p$-number. Moreover, if $P \nless \mathbf{C}_{G}\left(\mathbf{O}_{p}(G) \cap B\right)$, then $\left|G: \mathbf{C}_{G}(P \cap B)\right|$ is also a $p$-number, and each element in $(P \cap$ $A) \cup(P \cap B)$ has index a $p$-number. Therefore $G$ is $p$-decomposable by virtue of Lemma 5 , and $\left|G: \mathbf{C}_{G}(P)\right|$ is clearly a $p$-power. On the other hand, if $P \leqslant \mathbf{C}_{G}\left(\mathbf{O}_{p}(G) \cap B\right)$, Proposition 4 yields that $\mathbf{C}_{G}\left(\mathbf{O}_{p}(G) \cap B\right)$ is normal in $G$ and the quotient $G / \mathbf{C}_{G}\left(\mathbf{O}_{p}(G) \cap B\right)$ is an abelian $q$-group $(q \neq p)$. We deduce $G=\mathbf{C}_{G}(P \cap A) \mathbf{C}_{G}(P \cap B)$, and since $\mathbf{C}_{G}(P)=\mathbf{C}_{G}(P \cap A) \cap$ $\mathbf{C}_{G}(P \cap B)$, then $\left|G: \mathbf{C}_{G}(P)\right|=\left|G: \mathbf{C}_{G}(P \cap A)\right| \cdot\left|G: \mathbf{C}_{G}(P \cap B)\right|$ is a $\{p, q\}$-number.

Now we assume $P \nless \mathbf{F}(G)$. It cannot happen that both $P \cap A \nless \mathbf{F}(G)$ and $P \cap B \nless \mathbf{F}(G)$ by Proposition 5 (a). We may assume for instance that $P \cap A \notin \mathbf{F}(G)$ and $P \cap B \leqslant \mathbf{F}(G)$. By Proposition 5 (a) and (c), we get that $P \cap A \leqslant \mathbf{Z}(P)$ and $\left|G: \mathbf{C}_{G}(P \cap A)\right|$ is a $q$-number, where $q \neq p$. On the other hand, as $P$ is not abelian, $P \nless \mathbf{C}_{G}(P \cap B)$, so Lemma 7 yields $\left|G: \mathbf{C}_{G}(P \cap B)\right|$ is a $p$-number. Then $\left|G: \mathbf{C}_{G}(P)\right|$ is a $\{p, q\}$-number.

(2) Consider that $P=(P \cap A)(P \cap B) \in \operatorname{Syl}_{p}(G)$ is abelian. If $P \cap A \not \mathbf{F}(G)$ and $P \cap B \nless \mathbf{F}(G)$ then the claim follows from Proposition 5(d). Assume that $P \cap A \nless \mathbf{F}(G)$ and $P \cap B \leqslant \mathbf{F}(G)$, so $\left|G: \mathbf{C}_{G}(P \cap A)\right|$ is a $q$-number with $q \neq p$ by Proposition 5 (c). Since $P \leqslant \mathbf{C}_{G}\left(\mathbf{O}_{p}(G) \cap B\right)$, we deduce from Proposition 4 that $\mathbf{C}_{G}(P \cap B)$ is normal in $G$ with index an $r$-number $(r \neq p)$. If $q \neq r$, then $G=\mathbf{C}_{G}(P \cap A) \mathbf{C}_{G}(P \cap B)$ and the claim follows. If $q=r$, we obtain that $\left|\mathbf{C}_{G}(P \cap A): \mathbf{C}_{G}(P)\right|=\left|\mathbf{C}_{G}(P \cap A) \mathbf{C}_{G}(P \cap B): \mathbf{C}_{G}(P \cap B)\right|$ divides the $q$-number $\left|G: \mathbf{C}_{G}(P \cap B)\right|$. Hence the index $\left|G: \mathbf{C}_{G}(P)\right|$ is a $q$-power.

Now suppose $P=\mathbf{O}_{p}(G)$. Note that, by Proposition 4 , both subgroups $\mathbf{C}_{G}\left(\mathbf{O}_{p}(G) \cap A\right)$ and $\mathbf{C}_{G}\left(\mathbf{O}_{p}(G) \cap B\right)$ are normal in $G$ with indices a $q$-number and an $r$-number, respectively. The case $q \neq r$ is again clear. If $q=r$, the above reasoning on the index of $\left|\mathbf{C}_{G}(P \cap A): \mathbf{C}_{G}(P)\right|$ shows that $\left|G: \mathbf{C}_{G}(P)\right|$ is a $q$-power. Finally, from (1), (2) and Corollary 6 we conclude we last assertion of the theorem.

Now we end by proving Theorem $\mathrm{F}$, as a consequence of the previous result.

Proof of Theorem $\mathbf{E}$. The converse direction is trivial. Assume that $G=A B$ is a Baer factorisation, and let $P=(P \cap A)(P \cap B) \in \operatorname{Syl}_{p}(G)$ such that $P \cap A \in \operatorname{Syl}_{p}(A)$ and $P \cap B \in \operatorname{Syl}_{p}(B)$. Let $X \in\{A, B\}$. We claim that $\left|G: \mathbf{C}_{G}(P \cap X)\right|$ is a prime power. Now we distinguish two cases: either $P \cap X \nless \mathbf{F}(G)$ or $P \cap X=\mathrm{O}_{p}(G) \cap X$. In the first case, the claim follows from Proposition 5 (c). In the second case, if $P \leqslant \mathbf{C}_{G}\left(\mathbf{O}_{p}(G) \cap X\right)$, then we apply Proposition 4, and if $P \nless \mathbf{C}_{G}\left(\mathbf{O}_{p}(G) \cap X\right)$, then Lemma 7 follows.

Final examples. Some of the results stated in this section fail when the hypotheses are weakened to $p$-Baer factorisations, as the following examples show:

(1) Proposition 4 and Theorem $\mathrm{F}$ do not hold for $p$-Baer factorisations: Let $G=A B$ be the semidirect product of a non-abelian group $B$ of order 21 acting on an elementary abelian group $A$ of order 8 , in such a way that the subgroup of order 7 permutes the involutions transitively (this group appears in [7]). Then $i_{G}(g)=7$ for every 2-element $g \in G$ (i.e., $G$ is 2-Baer), and the unique abelian Sylow 2-subgroup $P$ of $G$ is equal to $A$, but $\left|G: \mathbf{C}_{G}(P)\right|=$ $\left|G: \mathbf{C}_{G}\left(\mathbf{O}_{2}(G) \cap A\right)\right|=21$.

(2) Proposition $\mathrm{D}$ does not hold either for $p$-Baer factorisations: Let $G$ be the group in (1). Then there is a subgroup $H$ of $G$ of order 24 and a subgroup $K$ of order 7 such that $G=H K$ is also a 2-Baer factorisation, and there exists a 2-element $x \in H$ such that $i_{H}(x)=3$, which clearly is not a 7 -number. 
(3) Proposition 5 (c) is neither true for $p$-Baer factorisations: Let now $Q$ be a cyclic group of order 7. Consider the regular wreath product $T=Q$ wr $G$ with $G$ the group in (1), and denote by $Q^{\natural}$ the basis group (we point out that this group appears in [5]). Then the factorisation $T=Q^{\natural} G$ is 2-Baer. Let $P$ be the Sylow 2-subgroup of $G$, so $P \in \operatorname{Syl}_{2}(T)$. Then $P=P \cap G \nless \mathbf{F}(T)$, but the index $\left|T: \mathbf{C}_{T}(P \cap G)\right|$ is divisible by 3 and 7 .

\section{References}

[1] B. Amberg, S. Franciosi, and F. de Giovanni. Products of groups. Oxford University Press Inc., 1992.

[2] R. BAER. "Group elements of prime power index". Trans. Amer. Math. Soc. 75 (1953) 20-47.

[3] A. Ballester-Bolinches, J. Cossey, AND Y. Li. "Mutually permutable products and conjugacy classes". Monatsh. Math. 170 (2013) 305-310.

[4] A. Ballester-Bolinches, R. Esteban-Romero, and M. AsaAd. Products of finite groups. Vol. 53. W. de Gruyter Expositions In Mathematics, 2010.

[5] Y. BERKOVICH AND L. S. KAZARIN. "Indices of elements and normal structure of finite groups". J. Algebra 283 (2005) 564-583.

[6] A. R. Camina And R. D. Camina. "Implications of conjugacy class size". J. Group Theory 1 (1998) 257-269.

[7] A. R. Camina, P. Shumyatsky, and C. Sica. "On elements of prime-power index in finite groups". J. Algebra 323 (2010) 522-525.

[8] D. Chillag and M. Herzog. "On the length of the conjugacy classes of finite groups". $J$. Algebra 131 (1990) 110-125.

[9] K. Doerk And T. Hawkes. Finite Soluble Groups. Vol. 4. W. de Gruyter Expositions In Mathematics, 1992.

[10] M. J. Felipe, A. Martínez-Pastor, and V. M. Ortiz-Sotomayor. "On finite groups with square-free conjugacy class sizes". Int. J. Group Theory 7 (2018) 17-24.

[11] H. Kurzweil And B. Stellmacher. The theory of finite groups: an introduction. SpringerVerlag, 2004.

[12] X. LiU, Y. WANG, AND H. WeI. "Notes on the length of conjugacy classes of finite groups". $J$. Pure Appl. Algebra 196 (2005) 111-117. 
It is a widely held opinion that the problem of classifying finite simple groups is close to a complete solution. This will certainly be one of the great achievements of mathematics of this century.

- NATHAN JACOBSON 



\title{
Zeros of irreducible characters in factorised groups
}

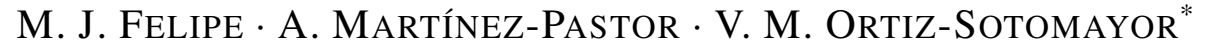

\begin{abstract}
An element $g$ of a finite group $G$ is said to be vanishing in $G$ if there exists an irreducible character $\chi$ of $G$ such that $\chi(g)=0$; in this case, $g$ is also called a zero of $G$. The aim of this paper is to obtain structural properties of a factorised group $G=A B$ when we impose some conditions on prime power order elements $g \in A \cup B$ which are (non-)vanishing in $G$.
\end{abstract}

Keywords Finite groups · Products of groups · Irreducible characters · Conjugacy classes - Vanishing elements

2010 MSC 20D40 $20 \mathrm{C} 15 \cdot 20 \mathrm{E} 45$

\section{Introduction}

Within finite group theory, the close relationship between character theory and the study of conjugacy classes is widely known. Regarding this last topic, several authors have investigated the connection between certain conjugacy class sizes (also called indices of elements) of a group $G$ and its structure. Further, recent results show up that the conjugacy classes of the elements in the factors of a factorised group exert a strong impact on the structure of the whole group (see [3], [10], [15], [16]).

In character theory, a celebrated Burnside's result asserts: every row in a character table of a finite group which corresponds to a non-linear complex character has a zero entry [17, Theorem 3.15]. Nevertheless, a non-central conjugacy class column may not contain a zero. This fact somehow violates the standard duality arising in many cases between the two referred research lines. Therefore, in [ $[\mathbf{1 8}]$ the authors introduce the next concept: an element $g \in G$ is vanishing in $G$ if there exists an irreducible character $\chi$ of $G$ such that $\chi(g)=0$ (in the literature, $g$ is also called a zero of $\chi$ ). Otherwise the element $g$ is said to be non-vanishing in $G$. As an immediate

\footnotetext{
*The first author is supported by Proyecto Prometeo II/2015/011, Generalitat Valenciana (Spain). The second author is supported by Proyecto MTM 2014-54707-C3-1-P, Ministerio de Economía, Industria y Competitividad (Spain), and by Proyecto Prometeo/2017/057, Generalitat Valenciana (Spain). The third author acknowledges the predoctoral grant ACIF/2016/170, Generalitat Valenciana (Spain).

Instituto Universitario de Matemática Pura y Aplicada (IUMPA-UPV), Universitat Politècnica de València, Camino de Vera s/n, 46022, Valencia, Spain.

凶: mfelipe@mat.upv.es, anamarti@mat.upv.es, vicorso@doctor.upv.es

ORCID iDs: 0000-0002-6699-3135, 0000-0002-0208-4098, 0000-0001-8649-5742
} 
consequence of the cited Burnside's result, we get that a group has no vanishing elements if and only if it is abelian. It is to be said that various questions concerning the (non-)vanishing elements of a group have been studied by numerous authors (in particular, those appearing as references in this paper).

It is therefore natural to wonder whether results based on conjugacy class sizes remain true if we restrict focus only to those indices that correspond to vanishing elements, i.e. if we consider only the so-called vanishing indices. In this spirit, some researchers have recently obtained positive results in certain cases. For instance, in 2010, Dolfi, Pacifici and Sanus proved that if a prime $p$ does not divide each vanishing index of a group $G$, then $G$ has a normal $p$-complement and abelian Sylow $p$-subgroups [13, Theorem A]. In 2016, Brough showed that for a fixed prime $p$ such that $(p-1,|G|)=1$, if all vanishing indices of $G$ are not divisible by $p^{2}$, then $G$ is soluble [7, Theorem A]. Moreover, if each vanishing index of $G$ is square-free, then $G$ is supersoluble [7, Theorem B]. The last two results turn to be the "vanishing versions" of [11. Theorem 1] and [11, Theorem 2], respectively. Besides, Brough and Kong have also showed in [8] that the hypotheses in the previous results can be weakened to vanishing indices of prime power order elements. We remark that the classification of finite simple groups (CFSG) is used in this development.

In this paper, we are interested in combining as a novelty the research on irreducible characters with the study of products of groups. More concretely, we want to analyse which information of a factorised group $G$ can be obtained from its character table when we consider the conjugacy classes in $G$ of elements in the factors. In particular, inspired by the aforementioned investigations, we deal with factorised groups having irreducible characters which evaluate zero on some elements in the factors. It is worthwhile to note that the product of two vanishing elements does not need to be vanishing in general. Moreover, an element in a (normal) subgroup can be vanishing in the whole group but not in that subgroup (see Example 3 ).

Focusing in products of groups, along the last decades, some relations of permutability between the factors have been considered by many authors, as for instance total permutability, mutual permutability (see [4]) and tcc-permutability (see [1], [2]). These last permutability relations are inherited by quotients, and they ensure the existence of a minimal normal subgroup contained in one of the factors. We are principally concerned about products of groups that satisfy both particular conditions, which we will name core-factorisations (see Definition 1).

In this framework, our purpose is to get a better understanding of how the vanishing elements in the factors control the structure of a group with a core-factorisation. Moreover, we will also deal with arithmetical conditions on the indices of those elements.

The paper is structured in the following way: Firstly, core-factorisations are defined in Section 2 and some properties of them, which will be crucial along the paper, are proved. In Section 3 , we analyse the case that a group with a core-factorisation has no vanishing $p$-elements in the factors for a prime $p$ (see Theorem 11. As a consequence, we obtain information of a factorised group when all prime divisors of its order are considered, that is, when there are no vanishing prime power order elements in the factors (see Corollary 3). Later on we obtain structural properties of groups with a core-factorisation from the vanishing indices in the whole group of some elements in the factors. Concretely, in Section 4 , we study the case when those vanishing indices are prime powers (Theorem 2 and Corollary 4 ). Next, we focus in Section 5 on the case that the indices are not divisible by a prime $p$ (see Theorem 3 ). The situation when those indices are square-free is also handled in this last section (see Theorems 4 and 7). In particular, we highlight that an affirmative answer to a question posed by Brough in [7] is given (Corollary 6). It is significant to mention again that all the previous results for core-factorisations will remain true when the factors are either totally, mutually or tcc-permutable (see Example 1). We remark that, in order to avoid repeating arguments from previous papers, when some proof runs as in 
the one of a known result with suitable changes, we refer to the corresponding one.

Throughout this paper, every group is assumed to be finite. The terminology here is as follows: for a group $G$ and an element $x \in G$, we call $i_{G}(x)$ the index of $x$ in $G$, that is, $i_{G}(x)=\left|G: \mathbf{C}_{G}(x)\right|$ is the size of the conjugacy class $x^{G}$. The set of prime divisors of the order of $G$ is denoted by $\pi(G)$. If $p$ is a prime, then $x \in G$ is a $p$-regular element if its order is not divisible by $p$. As customary, the set of all Sylow $p$-subgroups of $G$ is denoted by $\operatorname{Syl}_{p}(G)$, whilst $\operatorname{Hall}_{\pi}(G)$ is the set of all Hall $\pi$-subgroups of $G$ for a set of primes $\pi$. We write $\operatorname{Irr}(G)$ for the set of all irreducible complex characters of $G$. Given a group $G=A B$ which is the product of the subgroups $A$ and $B$, a subgroup $S$ is called prefactorised (with respect to this factorisation) if $S=(S \cap A)(S \cap B)$ (see [4]). We recall that a subgroup $U$ covers a section $V / W$ of a group $G$ if $W(U \cap V)=V$. The remaining notation is standard, and it is taken mainly from [12]. In particular, a normal subgroup $N$ of a group $G$ such that $N \neq G$ is denoted symbolically by $N \triangleleft G$. We also refer to [12] for details about classes of groups.

\section{Core-factorisations: definition and properties}

We analyse in this section the kind of factorisations we manage along the paper.

Definition 1. Let $1 \neq G=A B$ be the product of the subgroups $A$ and $B$. We say that $G=A B$ is a core-factorisation if for every proper normal subgroup $K$ of $G$ it holds that there exists a normal subgroup $1 \neq M / K$ of $G / K$ such that either $M / K \leqslant A K / K$ or $M / K \leqslant B K / K$ (i.e. either $A$ or $B$ covers $M / K)$.

Note that if we adopt the bar convention for the quotients over $K$, the above condition means that $\bar{A}_{\bar{G}} \bar{B}_{\bar{G}} \neq 1$, where $H_{X}$ denotes the core in a group $X$ of a subgroup $H$. This illustrates the given name for such factorisations.

Remark 1. Let us state some immediate facts:

1. If either $1 \neq G=A$ or $1 \neq G=B$, then $G=A B$ is always a core-factorisation.

2. If $G=A B$ is a core-factorisation of a simple group $G$, then either $G=A$ or $G=B$.

3. If we take $K=1$ in the above definition, then there exists a (minimal) normal subgroup of $G=A B$ contained in either $A$ or $B$.

We present now some non-trivial examples.

Example 1. Let $1 \neq G=A B$ be the product of the subgroups $A$ and $B$, and let assume that $A$ and $B$ satisfy one of the following permutability properties:

(i) $A$ and $B$ are mutually permutable, that is, $A$ permutes with every subgroup of $B$ and $B$ permutes with every subgroup of $A$.

(ii) $A$ and $B$ are tcc-permutable, that is, if for every subgroup $X$ of $A$ and every subgroup $Y$ of $B$, there exists $g \in\langle X, Y\rangle$ such that $X$ permutes with $Y^{g}$.

(iii) $A$ and $B$ are totally permutable, that is, every subgroup of $A$ permutes with every subgroup of $B$. (In particular, if this property holds, then $A$ and $B$ satisfy both (i) and (ii).)

Applying [4, Theorem 4.3.11] in (i) and [2, Lemma 2.5] in (ii), it can be seen that $A_{G} B_{G} \neq 1$. Also, the above permutability properties are clearly inherited by quotients. Thus $G=A B$ is a core-factorisation in all cases. We shall see later in Example 2 a group with a core-factorisation whose factors are neither mutually permutable nor tcc-permutable. 
Now we prove that the quotients of core-factorisations inherit the property.

Lemma 1. Let $G=A B$ be a core-factorisation, and let $M$ be a proper normal subgroup of $G$. Then $G / M=(A M / M)(B M / M)$ is also a core-factorisation.

Proof. We use the bar convention to denote the quotients over $M$. We take a normal subgroup $\bar{K} \triangleleft \bar{G}$, and we claim that there exists a normal subgroup $1 \neq \bar{N} / \bar{K}$ of $\bar{G} / \bar{K}$ covered by either $\bar{A}$ or $\bar{B}$. As $G=A B$ is a core-factorisation, then $G / K$ has a normal subgroup $1 \neq N / K$ such that either $N / K$ is covered by either $A$ or $B$. It follows

$$
1 \neq \frac{\bar{N}}{\bar{K}}=\frac{N / M}{K / M} \leqslant \frac{A K / M}{K / M}=\frac{(A M / M)(K / M)}{K / M}=\frac{\overline{A K}}{\bar{K}},
$$

or analogously the same is valid for $B$ instead of $A$.

The lemma below is a characterisation of core-factorisations via normal series.

Lemma 2. Let $1 \neq G=A B$ be the product of the subgroups $A$ and $B$. The following statements are pairwise equivalent:

(i) $G=A B$ is a core-factorisation.

(ii) There exists a normal series $1=N_{0} \unlhd N_{1} \unlhd \cdots \unlhd N_{n-1} \unlhd N_{n}=G$ such that either $N_{i} / N_{i-1} \leqslant A N_{i-1} / N_{i-1}$ or $N_{i} / N_{i-1} \leqslant B N_{i-1} / N_{i-1}$, for each $1 \leq i \leq n$ (i.e. $N_{i} / N_{i-1}$ is covered by either $A$ or $\left.B\right)$.

(iii) There exists a chief series $1=N_{0} \unlhd N_{1} \unlhd \cdots \unlhd N_{n-1} \unlhd N_{n}=G$ such that either $N_{i} / N_{i-1} \leqslant A N_{i-1} / N_{i-1}$ or $N_{i} / N_{i-1} \leqslant B N_{i-1} / N_{i-1}$, for each $1 \leq i \leq n$ (i.e. $N_{i} / N_{i-1}$ is covered by either $A$ or $\left.B\right)$.

Further, each term $N_{i}$ of such (chief) normal series is prefactorised and $N_{i}=\left(N_{i} \cap A\right)\left(N_{i} \cap B\right)$ is also a core-factorisation.

Proof. (i) implies (ii): Let $1 \neq N_{1} \unlhd G$ such that either $N_{1} \leqslant A$ or $N_{1} \leqslant B$, so $1 \triangleleft N_{1} \unlhd G$. Next, take $G / N_{1}=\left(A N_{1} / N_{1}\right)\left(B N_{1} / N_{1}\right)$. If $G / N_{1}=1$, then we have the desired series. If $1 \neq G / N_{1}$, then it is again a core-factorisation by the previous lemma. Therefore, there exists $1 \neq N_{2} / N_{1} \unlhd G / N_{1}$ such that either $N_{2} / N_{1} \leqslant A N_{1} / N_{1}$ or $N_{2} / N_{1} \leqslant B N_{1} / N_{1}$. So we get the series $1 \triangleleft N_{1} \triangleleft N_{2} \unlhd G$. Repeating this process until we reach a trivial quotient $G / N_{j}$, we get the desired series.

(ii) implies (iii): If we refine the series in (ii) to a chief series, then we get for each factor that there exist $N_{i}=T_{0} \unlhd T_{1} \unlhd T_{2} \unlhd \cdots \unlhd T_{k}=N_{i+1}$ such that each $T_{j} / T_{j-1}$ is a minimal normal subgroup of $G / T_{j-1}$. Let us see that either $T_{j} / T_{j-1} \leqslant A T_{j-1} / T_{j-1}$ or $T_{j} / T_{j-1} \leqslant$ $B T_{j-1} / T_{j-1}$. We may assume for instance $N_{i+1} \leqslant A N_{i}$. Thus $T_{j}=T_{j} \cap N_{i+1} \leqslant N_{i}\left(T_{j} \cap A\right) \leqslant$ $T_{j-1} A$, and so $T_{j} / T_{j-1} \leqslant A T_{j-1} / T_{j-1}$.

(iii) implies (i): We have to show that for each $K \triangleleft G$, there exists a non-trivial normal subgroup of $G / K$ covered by either $A$ or $B$. Let $1 \leq r \leq n$ be the minimum number such that $N_{r} \nless K$. Then $1 \neq N_{r} K / K$ is normal in $G / K$. We suppose for instance that $N_{r} / N_{r-1} \leqslant$ $A N_{r-1} / N_{r-1}$, so $N_{r} \leqslant A N_{r-1}$. By the minimality of $r$ it follows $N_{r} K / K \leqslant A K / K$.

Now we claim that each $N_{i}$ in such (chief) normal series is prefactorised, and we work by induction on $i$. The case $i=1$ is clear since either $N_{1} \leqslant A$ or $N_{1} \leqslant B$. Now we assume that $N_{i-1}=\left(N_{i-1} \cap A\right)\left(N_{i-1} \cap B\right)$ and we want to show that $N_{i}$ is also prefactorised. We may consider $N_{i} \leqslant A N_{i-1}$, and then $N_{i}=\left(N_{i} \cap A\right) N_{i-1}=\left(N_{i} \cap A\right)\left(N_{i-1} \cap A\right)\left(N_{i-1} \cap B\right) \subseteq$ $\left(N_{i} \cap A\right)\left(N_{i} \cap B\right) \subseteq N_{i}$. 
Fix a prefactorised $N_{i}=\left(N_{i} \cap A\right)\left(N_{i} \cap B\right)$ of a (chief) normal series of $G$ like in (ii) or (iii), for some $i \in\{1, \ldots, n\}$. We are showing that $N_{i}=\left(N_{i} \cap A\right)\left(N_{i} \cap B\right)$ is a core-factorisation. Consider the following portion of such (chief) normal series $1=N_{0} \unlhd N_{1} \unlhd \cdots \unlhd N_{i}$. Let $m \in$ $\{1, \ldots, i\}$. We claim that $N_{m}$ satisfies either $N_{m} \leqslant\left(N_{i} \cap A\right) N_{m-1}$ or $N_{m} \leqslant\left(N_{i} \cap B\right) N_{m-1}$ in order to apply the equivalence between (ii) and (i). We have by assumption that for instance $N_{m} \leqslant N_{m-1} A$, so $N_{m} \leqslant N_{m-1} A \cap N_{i}=N_{m-1}\left(A \cap N_{i}\right)$. The lemma is now established.

We point out that if $N$ is an arbitrary prefactorised normal subgroup of a core-factorisation $G=A B$, then $N=(N \cap A)(N \cap B)$ might not be a core-factorisation, as the next example shows.

Example 2. Consider $G=\operatorname{Sym}(4) \times\langle x\rangle$, where $\operatorname{Sym}(4)$ denotes the symmetric group of 4 letters and $o(x)=2$. If $A=\langle((1,2), x),((3,4), x),((1,3)(2,4), x)\rangle$ and $B=\langle((2,3,4), 1)$, $((3,4), 1),(1, x)\rangle$, then $G=A B$ is a core-factorisation, and $N=\operatorname{Sym}(4)=(N \cap A)(N \cap B)$ is not a core-factorisation, since there is no minimal normal subgroup of $N$ neither in $N \cap A$ nor in $N \cap B$. Moreover, it can be seen that $A$ and $B$ are not either mutually nor tcc-permutable.

\section{On vanishing elements}

The main objective of this section is to prove Theorem 1 and Corollary 3 . Let us state first some key ingredients for locating vanishing elements in a given group.

Lemma 3. [14, Lemma 2.9] Let $N \leqslant M \leqslant G$ be such that $N$ and $M$ are normal in $G$ and $(|N|,|M / N|)=1$. If $N$ is minimal normal in $G, \mathbf{C}_{M}(N) \leqslant N$ and $M / N$ is abelian, then every element in $M \backslash N$ is vanishing in $G$.

In 2017, Bianchi, Brough, Camina and Pacifici obtained the subsequent result.

Lemma 4. [5, Corollary 4.4] Let $G$ be a group, and $K$ an abelian minimal normal subgroup of $G$. Let $M / N$ be a chief factor of $G$ such that $(|K|,|M / N|)=1$, and $N=\mathbf{C}_{M}(K)$. Then every element of $M \backslash N$ is a vanishing element of $G$.

Let $p$ be a prime, and $\chi \in \operatorname{Irr}(G)$. Recall that $\chi$ is of $p$-defect zero if $p$ does not divide $\frac{|G|}{\chi(1)}$. A well-known result of Brauer [17. Theorem 8.17] highlights the significance that this property has for vanishing elements: if $\chi$ is an irreducible character of $p$-defect zero of $G$ then, for every $g \in G$ such that $p$ divides the order of $g$, it holds $\chi(g)=0$. The following lemma yields elements of normal subgroups that vanish in the whole group.

Lemma 5. [7, Lemma 2.2] Let $N$ be a normal subgroup of a group $G$. If $N$ has an irreducible character of p-defect zero, then every element of $N$ of order divisible by $p$ is a vanishing element in $G$.

We now focus on vanishing elements in simple groups. The combination of some results in [14], which use the classification, gives the following.

Proposition 1. Let $S$ be a non-abelian simple group, and let $p \in \pi(S)$. Then, either there exists $\chi \in \operatorname{Irr}(S)$ such that $\chi$ is of p-defect zero, or there exists a p-element $x \in S$ and $\chi \in \operatorname{Irr}(S)$ such that $\chi$ extends to $\operatorname{Aut}(S)$ and $\chi$ vanishes on $x$.

Proof. If either $S$ is a group of Lie type or $p \geq 5$, then [14. Proposition 2.1] applies and $S$ has an irreducible character of $p$-defect zero (note that this case includes the groups $A_{5} \cong P S L(2,5)$ and $A_{6} \cong P S L(2,9)$ ). Hence it remains to consider sporadic simple groups and alternating groups, and $p \in\{2,3\}$. Firstly, in virtue of [14, Lemma 2.3], for a sporadic simple group $S$ 
there exists always an irreducible character which extends to $\operatorname{Aut}(S)$ and it vanishes on a $p$ element. For alternating groups $A_{n}$ with $n \geq 7$, it is known by [14. Proposition 2.4] that $A_{n}$ has two irreducible characters $\chi_{2}, \chi_{3}$ such that $\chi_{2}$ vanishes on a 2-element and $\chi_{3}$ vanishes on an element of order 3 . Further, both $\chi_{2}$ and $\chi_{3}$ extend to $\operatorname{Aut}\left(A_{n}\right)$.

An argument included within the proof of [14, Theorem A] provides the following proposition, which turns to be essential in the remainder of the section.

Proposition 2. Let $N$ be a non-abelian minimal normal subgroup of a finite group $G$, and let $p \in \pi(N)$. Then there exists a p-element in $N$ which is vanishing in $G$.

Proof. We have that $N=S_{1} \times \cdots \times S_{k}$, where each $S_{i}$ is isomorphic to a non-abelian simple group $S$ with $p$ dividing its order. If $S$ has a character $\theta$ of $p$-defect zero, then $\chi:=\theta \times \cdots \times \theta \in$ $\operatorname{Irr}(N)$ and it is clear that $\chi$ is also of $p$-defect zero. Let $1 \neq x_{i} \in S_{i}$ be a $p$-element. Then $1 \neq x:=x_{1} \cdots x_{k} \in N$ is a $p$-element and Lemma 5 provides that $x$ is vanishing in $G$.

Let $i \in\{1, \ldots, k\}$ and suppose that $S_{i}$ does not have a character of $p$-defect zero. By Proposition 11, there exists $\theta \in \operatorname{Irr}\left(S_{i}\right)$ and a $p$-element $y_{i} \in S_{i}$ such that $\theta\left(y_{i}\right)=0$ (so $1 \neq y_{i}$ ) and $\theta$ extends to $\operatorname{Aut}\left(S_{i}\right)$. Thus $1 \neq y:=y_{1} \cdots y_{k} \in N$ is a $p$-element, and by [14, Proposition 2.2] it follows that $\chi:=\theta \times \cdots \times \theta \in \operatorname{Irr}(N)$ extends to $G$. Moreover, $\chi(y)=0$, and the result is now established.

From now on we deal with (non-)vanishing elements in factorised groups. The next example gives insight into occurring phenomena.

Example 3. Let $G=\operatorname{Sym}(4) \times\langle x\rangle=A B$ be the factorised group as in Example 2. Note that although $((3,4), x)$ is a vanishing element in $A$ and $((3,4), 1)$ is a vanishing element in $B$, the product $((3,4), x)((3,4), 1)=(1, x) \in \mathbf{Z}(G)$ and so it is non-vanishing in $G$. On the other hand, $((2,3,4), 1)$ is a non-vanishing element in $B$ which is vanishing in $G$.

Remark 2. We claim that the hypotheses regarding vanishing elements of the results stated from now on are inherited by every non-trivial quotient of a group $G$, where $G=A B$ is a core-factorisation. Indeed, let $N$ be a proper normal subgroup of $G$. Note that $G / N=$ $(A N / N)(B N / N)$ is also a core-factorisation by Lemma 1. Since there exists a bijection between $\operatorname{Irr}(G / N)$ and the set of all characters in $\operatorname{Irr}(G)$ containing $N$ in their kernel, if $x N \in$ $A N / N \cup B N / N$ is a vanishing (prime power order) element of $G / N$, then we can assume $x \in A \cup B$, and that $x$ is also a vanishing (prime power order) element of $G$. This fact will be used in the sequel, sometimes with no reference.

Our first significant result analyses core-factorisations with no vanishing $p$-elements in the factors. We remark that the CFSG is needed.

Theorem 1. Let $G=A B$ be a core-factorisation, and let $p$ be a prime. If every p-element in $A \cup B$ is non-vanishing in $G$, then $G$ has a normal Sylow p-subgroup.

Proof. Let $G$ be a counterexample of minimal order to the result, and take $P \in \operatorname{Syl}_{p}(G)$. Clearly we can assume that $\mathbf{O}_{p}(G)$ is proper in $G$. Hence by Remark 2 and the minimality of $G$ we may suppose $\mathbf{O}_{p}(G)=1$. Since $G=A B$ is a core-factorisation, we can consider a minimal normal subgroup $N$ of $G$ such that $N \leqslant A$, for instance. Let us suppose that $p$ divides its order. Then $N$ is non-abelian, and by Proposition 2 there is a $p$-element $x \in N$ which is vanishing in $G$, a contradiction. So $p$ does not divide the order of $N$. In particular, we may assume that $N$ is proper in $G$. By minimality and Remark 2 we obtain that $P N / N$ is normal in $G / N$, and then $G$ is $p$-separable. 
We can choose by Lemma 2 a chief series $1=N_{0} \unlhd N_{1}=N \unlhd \cdots \unlhd N_{n-1} \unlhd N_{n}=G$ such that each chief factor $N_{i} / N_{i-1}$ is covered by either $A$ or $B$. Let $j \in\{2, \ldots, n\}$ be the minimum number such that $p$ divides $\left|N_{j} / N_{j-1}\right|$. Then $N_{j} / N_{j-1}$ is a minimal normal subgroup of $G / N_{j-1}$ and it is $p$-elementary abelian. It follows that $N_{j} / N=N_{j-1} / N \times P_{0} / N$, where $1 \neq P_{0} / N=P N / N \cap N_{j} / N$ is the unique Sylow $p$-subgroup (and elementary abelian) of $N_{j} / N$. We claim that every element of $P_{0} \backslash N$ is vanishing in $G$. Note that $P_{0} / N$ is abelian and normal in $G / N$. It also holds $\left(|N|,\left|P_{0} / N\right|\right)=1$. In addition, since $N=\mathbf{O}_{p^{\prime}}\left(P_{0}\right)$ and $\mathbf{O}_{p}\left(P_{0}\right) \leqslant \mathbf{O}_{p}(G)=1$, then $\mathbf{C}_{P_{0}}(N) \leqslant N$. Lemma 3 yields that every element in $P_{0} \backslash N$ is vanishing in $G$. Therefore, it remains to find a $p$-element in $P_{0} \backslash N$ lying in either $A$ or $B$ in order to get the final contradiction.

Since $N_{j}=\left(N_{j} \cap A\right)\left(N_{j} \cap B\right)$ by Lemma 2, applying [15. Lemma 2] we can affirm that the unique Sylow $p$-subgroup $P_{0} / N$ of $N_{j} / N$ is also prefactorised, that is, $P_{0} / N=\left(P_{0} / N \cap\left(N_{j} \cap\right.\right.$ $A) / N)\left(P_{0} / N \cap\left(N_{j} \cap B\right) N / N\right)$. Let $X \in\{A, B\}$ such that $1 \neq P_{0} / N \cap\left(N_{j} \cap X\right) N / N=$ $\left(P_{0} \cap N_{j} \cap X\right) N / N$. If we pick a $p$-element $1 \neq x \in\left(P_{0} \cap N_{j} \cap X\right) \backslash N$, then $x$ is vanishing in $G$. Hence the result is established.

As an immediate consequence, when we take the trivial factorisation $G=A=B$ in the above theorem, we obtain [14, Theorem A]. In their proof, the authors apply Lemma 3 to the centre of a Sylow subgroup in order to get the final contradiction. We highlight that the centre subgroup may not be prefactorised (see [4, Example 4.1.43]) and so our reasonings differ.

Another consequence of Theorem 1 is the following.

Corollary 1. Let $G=A B$ be a core-factorisation, and let $\sigma$ be a set of primes. If every $\sigma$ element of prime power order in $A \cup B$ is non-vanishing in $G$, then $G$ has a nilpotent normal Hall $\sigma$-subgroup.

Proof. Apply Theorem 1 for each prime in $\sigma$.

Note that if $\sigma=p^{\prime}$ in the above result, then it generalises [14, Corollary B]. Indeed, the next corollary extends [14, Corollary $\mathrm{C}]$ for factorised groups.

Corollary 2. Let $G=A B$ be a core-factorisation, and let $\{p, q\} \subseteq \pi(G)$. If every element in $A \cup B$ vanishing in $G$ has order a $\{p, q\}$-number, then $G$ is soluble.

Proof. We denote by $\sigma:=\{p, q\}^{\prime}$. In virtue of Corollary 1 , $G$ has a nilpotent normal Hall $\sigma$-subgroup $N$. Now, $G / N$ is soluble because it is a $\{p, q\}$-group, so $G$ is also soluble.

If we consider the case when the hypotheses in Theorem 1 hold for all primes, then it follows clearly that those groups are nilpotent. But actually we obtain the stronger fact that they are abelian. The next result is essential in the proof of this fact.

Proposition 3. [18, Theorem B] If $G$ is supersoluble, then every element in $G \backslash \mathbf{Z}(\mathbf{F}(G))$ is vanishing in $G$. In particular, if $G$ is nilpotent, then all elements in $G \backslash \mathbf{Z}(G)$ are vanishing in $G$.

Corollary 3. Let $G=A B$ be a core-factorisation. The following statements are pairwise equivalent:

(1) Every element $x \in A \cup B$ is non-vanishing in $G$.

(2) Every prime power order element $x \in A \cup B$ is non-vanishing in $G$.

(3) $G$ is abelian. 
Proof. There is no doubt in the implications (1) $\Rightarrow(2)$ and (3) $\Rightarrow(1)$, so let us prove (2) $\Rightarrow$ (3). Clearly, by Theorem 1, $G$ is nilpotent. Since we are assuming that every prime power order element lying in $A \cup B$ is non-vanishing in $G$, then Proposition 3 provides that all Sylow subgroups of $A$ and $B$ are central in $G$, and thus $G=A B \leqslant \mathbf{Z}(G)$.

As it has been said before, from Burnside's result quoted in the introduction it is elementary to show that a group is abelian if and only if it has no vanishing elements. Indeed, it is enough to consider in this last characterisation only prime power order elements, as we directly deduce by taking the trivial factorisation in the previous corollary. This claim can be also obtained from [19. Theorem B], which asserts that a non-linear complex character vanishes on a prime power order element (it also uses the CFSG). In any case, both proofs emphasize the difficulty of handling only prime power order elements. Moreover, observe that [19. Theorem B] does not imply directly Corollary 3 , since we cannot assure in a factorised group that a vanishing prime power order element lies in one of the factors.

\section{Prime power vanishing indices}

In [9], Camina and Camina analysed the structure of the so-called $p$-Baer groups, i.e. groups all of whose $p$-elements have prime power indices for a given prime $p$. Next, in [15] we extended this study through products of two arbitrary groups. Thus, as stated in the introduction, it seems natural to address the corresponding vanishing problem, i.e. vanishing indices which are prime powers, in particular for factorised groups.

Let us enunciate first some preliminary results. The subsequent well-established one is due to Wielandt.

Lemma 6. Let $G$ be a finite group and $p$ a prime. If $x \in G$ is a p-element and $i_{G}(x)$ is a p-number, then $x \in \mathbf{O}_{p}(G)$.

In [9], Camina and Camina proved the next proposition, which extends both the above lemma and the celebrated Burnside's result about the non-simplicity of groups with a conjugacy class of prime power size.

Proposition 4. [9. Theorem 1] All elements of prime power index of a finite group G lie in $\mathbf{F}_{2}(G)$, the second term of the Fitting series of $G$.

The main result of $[\mathbf{6}]$ is the following one.

Proposition 5. Let $G$ be a group which contains a non-trivial normal $p$-subgroup $N$ for $p$ a prime. Then each $x \in N$ such that $p$ does not divide $i_{G}(x)$ is non-vanishing in $G$.

Finally, the lemma below is elementary.

Lemma 7. Let $N$ be a normal subgroup of a group $G$. We have:

(a) $i_{N}(x)$ divides $i_{G}(x)$, for any $x \in N$.

(b) $i_{G / N}(x N)$ divides $i_{G}(x)$, for any $x \in G$.

Remark 3. Note that, hereafter, in the results stated the arithmetical hypotheses on the indices are inherited by non-trivial quotients of core-factorisations. Indeed, let $G=A B$ be a corefactorisation and suppose for an element $x \in A \cup B$ that $i_{G}(x)$ is a prime power, square-free, or not divisible by a given prime, respectively. Since $i_{G / N}(x N)$ divides $i_{G}(x)$ by the above lemma, we get that $i_{G / N}(x N)$ is also a prime power, square-free, or not divisible by such prime, respectively. 
We are now ready to prove the following vanishing versions of [15. Theorem A (1-2)] and [15. Theorem B (1)] for core-factorisations, respectively. We emphasize that the techniques used in that approach are not valid when we work with zeros of irreducible characters.

Theorem 2. Let $G=A B$ be a core-factorisation. Let $p$ be a prime, and $P \in \operatorname{Syl}_{p}(G)$. Assume that every p-element $x \in A \cup B$ vanishing in $G$ has prime power index. Then:

(1) If all the considered indices are p-numbers, then $P$ is normal in $G$.

(2) $G / \mathbf{O}_{p^{\prime}}(\mathbf{F}(G))$ has a normal Sylow p-subgroup. So $G$ is p-soluble of p-length 1.

Proof. (1) If all the indices of vanishing $p$-elements $x \in A \cup B$ are $p$-numbers, then it is enough to reproduce the proof of Theorem 1. Notice that the contradictions now will be derived from Lemma 6 ,

(2) It is enough to show that $G / \mathbf{F}(G)$ has a normal Sylow $p$-subgroup, since it is isomorphic to $\left(G / \mathbf{O}_{p^{\prime}}(\mathbf{F}(G))\right) /\left(\mathbf{F}(G) / \mathbf{O}_{p^{\prime}}(\mathbf{F}(G))\right)$ and $\mathbf{F}(G) / \mathbf{O}_{p^{\prime}}(\mathbf{F}(G))$ is a $p$-group. Let us denote $\bar{G}:=$ $G / \mathbf{F}(G)$, and let us assume $\bar{G} \neq 1$. If the statement is false, then by Theorem 1 there exists a vanishing $p$-element $1 \neq \bar{x}=x \mathbf{F}(G)$ in $\bar{A} \cup \bar{B}$. By Remark 2, $x \notin \mathbf{F}(G)$ is a vanishing $p$ element in $A \cup B$, and so $i_{G}(x)$ is a power of a prime $q \neq p$. It follows $x \in \mathbf{F}_{2}(G)$ by Proposition 4. so $1 \neq \bar{x} \in \mathbf{O}_{p}(\bar{G})$. Proposition 5 implies that $p$ divides $i_{\bar{G}}(\bar{x})$, and so $p$ divides $i_{G}(x)$, a contradiction. Finally, the second assertion about the $p$-solubility of $G$ follows directly.

We remark that the vanishing analogue of [15. Theorem B (2)] is not true, that is, if the considered vanishing indices are powers of primes distinct from $p$, then the Sylow $p$-subgroup might not be abelian:

Example 4. Let $G$ be a Suzuki group of degree 8, and let $H$ be the normaliser of a Sylow 2subgroup of $G$. Then $H$ is a core-factorisation of its Sylow 2-subgroup and a Sylow subgroup of order 7 , and $H$ does not have vanishing 2-elements. Nevertheless, the Sylow 2-subgroup of $H$ is non-abelian.

Moreover, [15, Theorem B] asserts that if all the $p$-elements in a factor have prime power indices in the whole factorised group, then there is a unique prime that divides all the considered indices. However, we do not know if the vanishing version of this fact is true.

Finally, note that if we consider the assumptions in Theorem 2 for every prime in $\pi(G)$, then the third statement tells us that $G / \mathbf{F}(G)$ is nilpotent. In fact, the following result shows that $G / \mathbf{F}(G)$ is abelian for such a group (compare with [15, Corollary C (1)]).

Corollary 4. Let $G=A B$ be a core-factorisation. If every prime power order element $x \in$ $A \cup B$ vanishing in $G$ has prime power index, then $G / \mathbf{F}(G)$ is abelian. In particular, if these prime powers are actually p-numbers for a prime $p$, then $G$ has a normal Sylow p-subgroup and abelian Hall $p^{\prime}$-subgroups.

Proof. $G / \mathbf{F}(G)$ is nilpotent by Theorem 2 (3). Let us denote by $\bar{G}:=G / \mathbf{F}(G)$, and let us assume that $\bar{G} \neq 1$ and that there exists $1 \neq \bar{x}=x \mathbf{F}(G)$ a prime power order element in $\bar{A} \cup \bar{B}$ vanishing in $\bar{G}$. Then $\bar{x}$ is a $p$-element for some prime $p$, and we may suppose $x \in(A \cup B) \backslash \mathbf{F}(G)$ is a $p$-element vanishing in $G$. By assumption, we have that $i_{G}(x)$ is a prime power. Since $\bar{G}$ is nilpotent, then by Proposition 5 it follows that $i_{\bar{G}}(\bar{x})$ is a $p$-number, and so is $i_{G}(x)$. It follows by Wielandt's lemma that $x \in \mathbf{O}_{p}(G)$, so $\bar{x}=1$, a contradiction. Thus $\bar{G}$ does not have any vanishing prime power order element in $\bar{A} \cup \bar{B}$, and by Corollary 3 we get that it is abelian.

For the second assertion, note that $P$ is the unique Sylow $p$-subgroup of $G$ by Theorem 2 (1), so we claim that $H \cong G / P$ is an abelian Hall $p^{\prime}$-subgroup of $G$. Let us denote $\tilde{G}:=G / P$, 
so $\tilde{G}=\tilde{A} \tilde{B}$. Hence, $\tilde{G}$ does not have any vanishing prime power order element in $\tilde{A} \cup \tilde{B}$, since otherwise those elements are central by our assumptions, a contradiction. So it follows by Corollary 3 that $\tilde{G}=G / P \cong H$ is abelian.

\section{Square-free vanishing indices}

In this last section we focus on vanishing indices in factorised groups which are square-free, motivated by previous developments in $[\mathbf{7}],[\overline{\mathbf{8}}]$ and $[\mathbf{1 3}]$. Our first theorem treats the case when the vanishing indices are not divisible by a fixed prime $p$. We should comment that, although some arguments in the proof of the first statement are similar to those in [8. Theorem 3.3], we include them here for the sake of comprehensiveness.

Theorem 3. Let $G=A B$ be a core-factorisation.

(1) Assume that $p$ does not divide $i_{G}(x)$ for every p-regular element of prime power order $x \in A \cup B$ vanishing in $G$. Then $G$ is p-nilpotent.

(2) If $p$ does not divide $i_{G}(x)$ for every prime power order element $x \in A \cup B$ vanishing in $G$, then $G$ is p-nilpotent with abelian Sylow p-subgroups.

Proof. (1) Assume the result is false. We argue with $G$ a minimal counterexample to the theorem. By minimality, we may suppose that $\mathbf{O}_{p^{\prime}}(G)=1$. Let $N$ be a minimal normal subgroup of $G$ such that $N \leqslant A$, for instance. If $N$ is soluble, since $p$ divides its order it follows that $N$ is a $p$-group. We can assume that $N$ is proper in $G$ since otherwise $G$ is a $p$-group, so by minimality we get that $G / N$ is $p$-nilpotent. Hence $G$ is $p$-separable, and $\mathbf{C}_{G}\left(\mathbf{O}_{p}(G)\right) \leqslant \mathbf{O}_{p}(G)$. This last fact and our assumptions produce that there are no $p$-regular elements of prime power order $x \in A \cup B$ vanishing in $G$, and Corollary 1 applies with $\sigma=p^{\prime}$. Thus $N$ is non-soluble, and applying the same arguments as in the second paragraph in the proof of [8, Theorem 3.3], it can be obtained a $p$-regular element of prime power order in $N \leqslant A$ which is vanishing in $G$ and whose conjugacy class size in $G$ is divisible by $p$, the final contradiction.

(2) $G$ is $p$-nilpotent by (1). Let us denote $\tilde{G}:=G / H$ where $H$ is the unique Hall $p^{\prime}$-subgroup of $G$, and then $\tilde{G}=\tilde{A} \tilde{B}$. Then, $\tilde{G}$ does not have any vanishing prime power order element in $\tilde{A} \cup \tilde{B}$, because otherwise the hypotheses imply that those elements are central, a contradiction. Now in virtue of Corollary 3 we get that $\tilde{G}$ is abelian.

Note that Theorem 3 provides a vanishing version of $[\mathbf{3}$. Theorem 1.1] for products of two groups, even relaxing the mutual permutability of the factors. We also remark that $[\mathbf{8}$. Theorem 3.3] is Theorem 3 (1) for the trivial factorisation. Indeed, (2) implies the next corollary, which improves the main result of [13] by considering only vanishing indices of prime power order elements:

Corollary 5. Let $G$ be a group, and $p$ be a prime. If $p$ does not divide any vanishing index of a prime power order element, then $G$ is p-nilpotent with abelian Sylow p-subgroups.

Regarding square-free vanishing indices, we first analyse those which are not divisible by $p^{2}$, for a fixed prime $p$. The next proposition is actually the vanishing version of [16. Theorem A]. We point out that this result is valid for any arbitrary factorisation of a $p$-group.

Proposition 6. Let $p$ be a prime number and let $P=A B$ be a p-group such that $p^{2}$ does not divide $i_{P}(x)$ for all $x \in A \cup B$ vanishing in $P$. Then $P^{\prime} \leqslant \mathbf{\Phi}(P) \leqslant \mathbf{Z}(P)$, $P^{\prime}$ is elementary abelian and $\left|P^{\prime}\right| \leq p^{2}$. 
Proof. Since the non-vanishing elements of a $p$-group lie in its centre because of Proposition 3 . we can apply directly [16. Theorem A] in order to get the thesis.

The following lemma will be essential in the sequel.

Lemma 8. [3, Lemma 2.4] Let $p$ be a prime, and $Q$ be a $p^{\prime}$-group acting faithfully on an elementary abelian $p$-group $N$ with $|[x, N]|=p$, for all $1 \neq x \in Q$. Then $Q$ is cyclic.

In [7], the author posed the following question: a group such that all its vanishing indices are not divisible by $p^{2}$, for a prime satisfying $(p-1,|G|)=1$, must be $p$-nilpotent? The following theorem gives a positive answer to this question, even for some factorised groups (see Corollary 6 for the case $G=A=B$ ).

Theorem 4. Let $G=A B$ be a core-factorisation, and let $p$ be a prime such that $(p-1,|G|)=1$. Suppose that $i_{G}(x)$ is not divisible by $p^{2}$ for every prime power order element $x \in A \cup B$ vanishing in G. It follows that:

(1) $G$ is soluble.

(2) $G$ is p-nilpotent.

(3) If $P \in \operatorname{Syl}_{p}(G)$, then $P^{\prime} \leqslant \boldsymbol{\Phi}(P) \leqslant \mathbf{Z}(P)$, $P^{\prime}$ is elementary abelian and $\left|P^{\prime}\right| \leq p^{2}$.

Proof. (1) Suppose that the result is false and let $G$ be a counterexample of minimal order. Since every group of odd order is soluble, we may assume that $p=2$ because $(p-1,|G|)=1$. The class of soluble groups is a saturated formation, so we can suppose that there exists a unique minimal normal subgroup $N$. Moreover, $N$ is non-soluble. We have for instance $N \leqslant A$, because $G=A B$ is a core-factorisation. Then it is enough to reproduce the arguments in the proof of [8 , Theorem 3.1] to obtain a prime power order element in $N \leqslant A$ which is vanishing in $G$ and whose conjugacy class size is divisible by 4 , a contradiction.

(2) Assume that the result is not true and let $G$ be a counterexample of least possible order. By the minimality of $G$ we may suppose that $\mathbf{O}_{p^{\prime}}(G)=1$. Let $N$ be a minimal normal subgroup of $G$. Thus $p$ divides its order and, since $G$ is soluble by (1), then $N$ is $p$-elementary abelian. Moreover, the class of $p$-nilpotent groups is a saturated formation, so $N$ is the unique minimal normal subgroup of $G$ and by [12, A - 15.6, 15.8] we get $N=\mathbf{O}_{p}(G)=\mathbf{F}(G)=\mathbf{C}_{G}(N)$. We can consider $N \leqslant A$, for instance. We take $K / N$ a minimal normal subgroup of $G / N$ such that it is covered by either $A$ or $B$. We claim that each element in $K \backslash N$ is vanishing in $G$. Since $N=\mathbf{O}_{p}(G)$, then $K / N$ is $q$-elementary abelian for some prime $q \neq p$. Indeed, we get $\mathbf{C}_{K}(N) \leqslant \mathbf{C}_{G}(N)=N$. It follows by Lemma 3 that every element in $K \backslash N$ is vanishing in $G$.

Note that $K=[N] Q$ where $Q \in \operatorname{Syl}_{q}(K)$ is elementary abelian. If we take $1 \neq x N \in$ $K / N$, then we can assume that $x \in K \backslash N$ is a $q$-element in $A \cup B$ by conjugation. Hence $p^{2}$ does not divide $i_{G}(x)=\left|G: \mathbf{C}_{G}(x)\right|$. Note that the $p$-number $1 \neq\left|N: \mathbf{C}_{N}(x)\right|$ divides $i_{G}(x)$. On the other hand, $x$ acts coprimely on $N$, which is abelian, so $N=\mathbf{C}_{N}(x) \times[N, x]$. It follows $|[N, x]|=p$. Observe that $\mathbf{C}_{Q}(N)=Q \cap \mathbf{C}_{G}(N)=1$, so $Q$ acts faithfully and coprimely on $N$. Further, if $1 \neq y \in Q$, then $y \in K \backslash N$ and by the previous argument we get $|[N, y]|=p$. Now Lemma 8 leads to the fact that $Q$ is cyclic, so $|K / N|=q$ and $K=N\langle x\rangle$. Hence $\mathbf{C}_{N}(x)=\mathbf{C}_{N}(K)$ is normal in $G$. Since $\mathbf{C}_{N}(x)<N$, by the minimality of $N$ we obtain $\mathbf{C}_{N}(x)=1$ and so $N=[N, x]$ has order $p$. Now $G / N=\mathbf{N}_{G}(N) / \mathbf{C}_{G}(N)$ is isomorphich to a subgroup of $\operatorname{Aut}(N)$, which is isomorphic to $C_{p-1}$. It follows that $|G / N|$ divides both $p-1$ and $|G|$, the final contradiction.

(3) Notice that $P \in \operatorname{Syl}_{p}(G)$ is isomorphic to $G / \mathbf{O}_{p^{\prime}}(G)$ by the previous assertion. Hence the result follows by Proposition 6 . 
Corollary 6. Let $G$ be a group, and let $p$ be a prime such that $(p-1,|G|)=1$. Assume that $p^{2}$ does not divide $i_{G}(x)$ for each prime power order element $x$ vanishing in $G$. Then $G$ is a soluble p-nilpotent group. Moreover, if $P \in \operatorname{Syl}_{p}(G)$, then $P^{\prime} \leqslant \Phi(P) \leqslant \mathbf{Z}(P), P^{\prime}$ is elementary abelian and $\left|P^{\prime}\right| \leq p^{2}$.

In [16, Theorem B (c)] it is proved the following: "Let $G=A B$ be the product of the mutually permutable subgroups $A$ and $B$. Let $p$ be a fixed prime satisfying $(p-1,|G|)=1$. If all $p$-regular prime power order elements in $A \cup B$ have $i_{G}(x)$ not divisible by $p^{2}$, then $G / \mathbf{O}_{p}(G)$ has elementary abelian Sylow $p$-subroups". We point out that this property does not remain true under the hypotheses of Theorem 4 , as the following example shows:

Example 5. Let $G=[A] B$ be the semidirect product of a cyclic group $B$ of order 4 which acts transitively on a cyclic group $A$ of order 5 . Let the prime $p=2$. Then $G=A B$ is a corefactorisation, and all the vanishing elements of $G$ (not only those lying in $A \cup B$ ) have index not divisible by 4 . However, $\mathbf{O}_{2}(G)=1$ and $G / \mathbf{O}_{2}(G)$ does not have elementary abelian Sylow 2-subgroups.

We highlight that the arguments used in [16. Theorem C] can be generalised in order to obtain the following more general result for core-factorisations.

Theorem 5. Let $G=A B$ be a core-factorisation, and let $p$ be a prime. Suppose that for every prime power order p-regular element $x \in A \cup B$ vanishing in $G, i_{G}(x)$ is not divisible by $p^{2}$. If $G$ is p-soluble, then $G$ is p-supersoluble.

Proof. It is sufficient to follow the proof of [16. Theorem C]. Notice that, in this case, we can use Lemma 4 with a minimal normal subgroup $Z / N$ of $G / N$ such that lies in either $A N / N$ or $B N / N$. Thus, we can affirm that every element in $Z \backslash N$ is vanishing in $G$, in order to apply the assumption that $i_{G}(x)$ is not divisible by $p^{2}$ for every prime power order $p$-regular element $x \in Z \backslash N$.

When we consider square-free indices for all primes, we will derive some information for groups with a core-factorisation (Theorem 7) from the next more general result:

Theorem 6. Let $G=A B$ be the product of the subgroups $A$ and $B$, and assume that $G$ is supersoluble. Suppose that $i_{G}(x)$ is square-free for every prime power order element $x \in A \cup B$ vanishing in $G$. Then:

(1) $G^{\prime}$ is abelian.

(2) $G^{\prime}$ has elementary abelian Sylow subgroups.

(3) $\mathbf{F}(G)^{\prime}$ has Sylow p-subgroups of order at most $p^{2}$, for each prime $p$.

Proof. We adapt the proof of [16. Theorem D] for our hypotheses regarding vanishing elements.

To prove either (1) or (2), arguing by minimal counterexample in each case we can assume that there exists a prime $p$ such that $\mathbf{F}(G)=\mathbf{O}_{p}(G)=P$ is a Sylow $p$-subgroup of $G$. Since $G$ is supersoluble, then Proposition 3 yields that every $q$-element $(q \neq p) x \in A \cup B$ is vanishing in $G$. Thus we can apply for such an element the class size hypothesis.

We claim that $P$ satisfies the hypotheses in Proposition 6 . Note that $P=(P \cap A)(P \cap B)$ in virtue of [16. Lemma 2]. If we take a $p$-element $x \in(P \cap A) \cup(P \cap B)$ vanishing in $P$, then it follows that $x$ is vanishing in $G$; otherwise we get by Proposition 3 that $x \in \mathbf{Z}(\mathbf{F}(G))=\mathbf{Z}(P)$ which is impossible. Therefore $i_{P}(x)$ divides $i_{G}(x)$ which is square-free. Now Proposition 6 applies, so $P^{\prime} \leqslant \boldsymbol{\Phi}(P) \leqslant \mathbf{Z}(P)$ with $P^{\prime}$ elementary abelian of order at most $p^{2}$. By [16. Lemma 2], we may take $H=(H \cap A)(H \cap B)$ a Hall $p^{\prime}$-subgroup of $G$ such that $H \cap A \in \operatorname{Hall}_{p^{\prime}}(A)$ and $H \cap B \in \operatorname{Hall}_{p^{\prime}}(B)$. Note that we can assume either $H \cap A \neq 1$ or $H \cap B \neq 1$. If 
$x \in H \cap A$ (or $x \in H \cap B$ ) is a prime power order element, then $x$ is vanishing in $G$ and so $i_{G}(x)$ is square-free. Arguing as in the second paragraph of the proof of [16. Theorem D (1)], we deduce that $P^{\prime} \leqslant \Phi(P) \leqslant \mathbf{Z}(G)$, and so $\boldsymbol{\Phi}(G) \neq 1 \neq \mathbf{Z}(G)$.

We can also argue as in the third paragraph of the proof of [16. Theorem D (1)] to deduce that $H \cap A$ and $H \cap B$ are cyclic subgroups, since the class size hypotheses are applied to $q$ elements in $A \cup B$ which are vanishing in $G$. Let $H \cap A=\langle\alpha\rangle$. Following the proof of [16. Theorem D (1)], we can assume that for every prime $q(q \neq p)$, if $\alpha_{q}$ is the $q$-part of $\alpha$, then $N:=P\left\langle\alpha_{q}\right\rangle<G$, and $N=(N \cap A)(N \cap B)$ is normal in $G$. Let us see that $N$ inherits the hypotheses of the theorem. First, $N$ is clearly supersoluble. If a $p$-element $x \in(N \cap A) \cup(N \cap B)$ is vanishing in $N$ but not in $G$, then by Proposition 3 we obtain $x \in \mathbf{Z}(\mathbf{F}(G))=\mathbf{Z}(P)$, and so Proposition 5 leads to the fact that $x$ is non-vanishing in $N$, a contradiction. Moreover, all the $q$-elements $(q \neq p)$ in $(N \cap A) \cup(N \cap B)$ are vanishing in $G$ by the first paragraph. Thus, in both cases, $i_{N}(x)$ divides $i_{G}(x)$ which is square-free. Then we get a contradiction with the same arguments as in the proof of [16. Theorem D (1)] to prove the statement (1).

The assertion (2) can be obtained similarly as in the proof of [16. Theorem D (2)] since the hypotheses are applied to $q$-elements in $A \cup B$ which are vanishing in $G$.

The proof of the statement (3) runs as the one of [16. Theorem D (3)], but applying Proposition 6, which is the vanishing version of [16, Theorem A].

Theorem 7. Let $G=A B$ be a core-factorisation. Suppose that $i_{G}(x)$ is square-free for every prime power order element $x \in A \cup B$ vanishing in $G$. Then:

(1) $G$ is supersoluble.

(2) $G^{\prime}$ is abelian.

(3) $G^{\prime}$ has elementary abelian Sylow subgroups.

(4) $\mathbf{F}(G)^{\prime}$ has Sylow p-subgroups of order at most $p^{2}$, for each prime $p$.

Proof. (1) Considering the smallest prime divisor of $|G|$ and Theorem 4 (1), we conclude that $G$ is soluble. Hence, it is $p$-soluble for each prime $p$. Applying Theorem 5, we get that $G$ is $p$-supersoluble for each prime $p$, so it is supersoluble.

(2-4) These assertions follow from the previous theorem.

In [8, Theorem 3.2] the author gives a supersolubility criterion for a group such that every vanishing index of a prime power order element is square-free. We want to highlight that the following consequence of Theorem 7 gives more information on the structure of such a group. Moreover, our techniques differ from those used in [8, Theorem 3.2].

Corollary 7. Let $G$ be a group, and let assume that $i_{G}(x)$ is square-free for each prime power order element $x$ vanishing in $G$. Then $G$ is supersoluble, and $G^{\prime}$ is abelian with elementary abelian Sylow subgroups. Further, $\mathbf{F}(G)^{\prime}$ has Sylow p-subgroups of order at most $p^{2}$, for each prime $p$.

\section{References}

[1] M. Arroyo-Jordá, P. Arroyo-Jordá, A. Martínez-Pastor, And M.D. PÉrez-Ramos. "On conditional permutability and factorized groups". Ann. Mat. Pura Appl. 193 (2014) 11231138.

[2] M. Arroyo-Jordá, P. Arroyo-Jordá, And M.D. PÉrez-RAmos. “On conditional permutability and saturated formations”. Proc. Edinb. Math. Soc. 54 (2011) 309-319.

[3] A. Ballester-Bolinches, J. Cossey, And Y. Li. "Mutually permutable products and conjugacy classes". Monatsh. Math. 170 (2013) 305-310. 
[4] A. Ballester-Bolinches, R. Esteban-Romero, And M. As Aad. Products of finite groups. Vol. 53. W. de Gruyter Expositions In Mathematics, 2010.

[5] M. Bianchi, J. Brough, R.D. Camina, And E. Pacifici. "On vanishing class sizes in finite groups". J. Algebra 489 (2017) 446-459.

[6] J. Brough. "Non-vanishing elements in finite groups". J. Algebra 460 (2016) 387-391.

[7] J. BRough. "On vanishing criteria that control finite group structure". J. Algebra 458 (2016) 207215 .

[8] J. Brough AND Q. Kong. "On vanishing criteria that control finite group structure II". Bull. Aust. Math. Soc. 98 (2018) 251-257.

[9] A. R. CAminA AND R. D. CAminA. "Implications of conjugacy class size”. J. Group Theory 1 (1998) 257-269.

[10] J. COSSEY AND Y. LI. "On the structure of a mutually permutable product of finite groups". Acta Math. Hungar. 154 (2018) 525-529.

[11] J. Cossey And Y. WAng. "Remarks on the length of conjugacy classes of finite groups". Comm. Algebra 27 (1999) 4347-4353.

[12] K. Doerk And T. HaWkes. Finite Soluble Groups. Vol. 4. W. de Gruyter Expositions In Mathematics, 1992.

[13] S. DOLFI, E. PACIFICI, AND L. SANUS. "Groups whose vanishing class sizes are not divisible by a given prime”. Arch. Math. (Basel) 94 (2010) 311-317.

[14] S. Dolfi, E. PACIFICI, L. SANUS, AND P. SPIGA. "On the orders of zeros of irreducible characters". J. Algebra 321 (2009) 345-352.

[15] M. J. Felipe, A. Martínez-Pastor, And V. M. Ortiz-Sotomayor. "Prime power indices in factorised groups". Mediterr. J. Math. 14.6 (2017) article: 225.

[16] M. J. Felipe, A. Martínez-Pastor, And V. M. Ortiz-Sotomayor. "Square-free class sizes in products of groups". J. Algebra 491 (2017) 190-206.

[17] I. M. Is AACS. Character theory of finite groups. Academic Press Inc., 1976.

[18] I. M. ISAACS, G. NAVARro, AND T. R. WOLF. "Finite group elements where no irreducible character vanishes". J. Algebra 222 (1999) 413-423.

[19] G. Malle, G. Navarro, and J.B. Olson. "Zeros of characters of finite groups". J. Group Theory 3 (2000) 353-368. 
In a certain sense the subject of group theory is built up out of three basic concepts: that of a homomorphism, that of a normal subgroup, and that of the factor group of a group by a normal subgroup.

— IsRael Nathan Herstein 



\title{
Products of groups and class sizes of $\pi$-elements
}

\author{
M. J. FELIPE · A. MARTÍNEZ-PASTOR · V. M. ORTIZ-SOTOMAYOR*
}

\begin{abstract}
We provide structural criteria for some finite factorised groups $G=A B$ when the conjugacy class sizes in $G$ of certain $\pi$-elements in $A \cup B$ are either $\pi$-numbers or $\pi^{\prime}$-numbers, for a set of primes $\pi$. In particular, we extend for products of groups some earlier results.
\end{abstract}

Keywords Finite groups $\cdot$ Products of groups $\cdot$ Conjugacy classes $\cdot \pi$-structure

2010 MSC 20D10 $20 \mathrm{D} 40 \cdot 20 \mathrm{E} 45 \cdot 20 \mathrm{D} 20$

\section{Introduction}

Along the last decades, numerous researchers have investigated groups which can be factorised as the product of two subgroups. In this setting, one of the main goals is to study the influence that the structure of the factors has on the structure of the whole group (and vice versa). In some occasions, the imposition of certain permutability conditions on the subgroups in the factorisation has proved to be useful. A detailed account on this topic can be found in the book [3]. Throughout this paper, we deal with the so-called core-factorisations, introduced in [11] (see also Definition 1).

On the other hand, a current activity shows up that, in a factorised group, the sizes of the conjugacy classes in the group of the elements in the factors have a strong impact on the structure of the whole group (see, for instance, [2, 10, 11, 14]). Our main purpose here is to study the $\pi$-structure of groups with a core-factorisation when the class lengths in the group of the $\pi$ elements in the factors are either $\pi$-numbers or $\pi^{\prime}$-numbers, for a set of primes $\pi$. In fact, we present alternative proofs of some earlier results as consequences of our theorems when

\footnotetext{
${ }^{*}$ The first author is supported by Proyecto Prometeo II/2015/011, Generalitat Valenciana (Spain). The second author is supported by Proyecto MTM 2014-54707-C3-1-P, Ministerio de Economía, Industria y Competitividad (Spain), and by Proyecto Prometeo/2017/057, Generalitat Valenciana (Spain). The results in this paper are part of the third author's Ph.D. thesis, and he acknowledges the predoctoral grant ACIF/2016/170, Generalitat Valenciana (Spain). The authors are also supported by Proyecto PGC2018-096872-B-I00, Ministerio de Ciencia, Innovación y Universidades.
}

Instituto Universitario de Matemática Pura y Aplicada (IUMPA-UPV), Universitat Politècnica de València, Camino de Vera s/n, 46022, Valencia, Spain.

凶: mfelipe@mat.upv.es, anamarti@mat.upv.es, vicorso@doctor.upv.es

ORCID iDs: 0000-0002-6699-3135, 0000-0002-0208-4098, 0000-0001-8649-5742 
trivial factorisations are considered. We point out that Dolfi $([\overline{\mathbf{8}}])$ considered class lengths which are either $\pi$-numbers or $\pi^{\prime}$-numbers in a (not necessarily factorised) group, but focusing on all its elements (not only on those with order a $\pi$-number). It is worth also to highlight that, although some results on class sizes could be proved through elementary arguments, the analysis of the considered class size properties in the context of products of groups may need a wider approach, even for core-factorisations. Indeed, in a core-factorisation, there is no relation in general between the class size of an element in one factor and the size of the corresponding conjugacy class in the whole group, in contrast to other developments (see, for example, [14]).

The paper is structured in the following way: in Section 2 we gather the definition and some properties of core-factorisations. Later on, in Section 3 we analyse groups with a corefactorisation such that the class lengths in the whole group of $\pi$-elements of prime power order in the factors are $\pi$-numbers (Theorem A. Then, in Section 4 , we put focus on groups with a corefactorisation whose $\pi$-elements (not necessarily of prime power order) in the factors have class sizes equal to either $\pi$-numbers or $\pi^{\prime}$-numbers (Theorem B, and Corollary C for not necessarily factorised groups). We also analyse in Theorem $\mathrm{D}$ this last condition on the class sizes of every element in the factors. Finally, as a consequence of the previous results, prime power class sizes are studied for the $\pi$-elements in the factors of a group with a core-factorisation (Theorem E). We want to remark that, along the whole paper, we provide numerous examples which show the scope of the results presented.

In the sequel, all groups under consideration are finite. For a group $G$ and an element $x \in G$, the conjugacy class of $x$ in $G$ is $x^{G}$, and its size is $\left|x^{G}\right|=\left|G: \mathbf{C}_{G}(x)\right|$. We denote the set of all prime divisors of a natural number $n$ by $\pi(n)$, and in particular we use $\pi(G)$ for the set of all prime divisors of the order of $G$. The set of all Hall $\pi$-subgroups of $G$ is expressed by $\operatorname{Hall}_{\pi}(G)$, where $\pi$ will always denote a set of primes. A group such that $G=\mathbf{O}_{\pi}(G) \times \mathbf{O}_{\pi^{\prime}}(G)$ is said to be $\pi$-decomposable. By $\operatorname{core}_{X}(H)$ we mean the core in a group $X$ of a subgroup $H$, i.e. the largest normal subgroup of $X$ contained in $H$. Given a group $G=A B$ which is the product of the subgroups $A$ and $B$, a subgroup $S$ is called prefactorised (with respect to this factorisation) if $S=(S \cap A)(S \cap B)$ (see [1] ). We recall that a subgroup $U$ covers a section $V / W$ of a group $G$ if $W(U \cap V)=V$. As usual, CFSG will denote the classification of finite simple groups. The remaining notation and terminology is standard within the theory of groups, and it is taken mainly from [7]. We also refer to this book for details about classes of groups.

\section{On core-factorisations}

As mentioned in the introduction, along the paper we deal with core-factorisations. We start this section by introducing that concept. Besides our initial inspiration in [11] within the framework of products of groups with certain permutability conditions on the factors, this notion can be also motivated by the following observation: If $\pi$ is a set of primes and $G$ is a group that possesses both Hall $\pi$-subgroups and Hall $\pi^{\prime}$-subgroups, say $H$ and $L$ respectively, then $G=H L$ is a $\pi$-separable group if and only if for a chief series of $G$ it holds that all the chief factors are covered by either $H$ or $L$.

Definition 1. Let $1 \neq G=A B$ be the product of the subgroups $A$ and $B$. We say that $G=A B$ is a core-factorisation whenever $G$ possesses a chief series such that each chief factor of $G$ is covered by either $A$ or $B$.

We point out that this definition of a core-factorisation is equivalent to that given in [11] (see Lemma 1 below). Next we collect some of its properties, some of which appear in the cited paper. 
Remark 1. Let us state some facts:

(i) If either $1 \neq G=A$ or $1 \neq G=B$, then $G=A B$ is always a core-factorisation.

(ii) If $G=A B$ is a core-factorisation, then there exists always a minimal normal subgroup of $G$ contained in either $A$ or $B$.

(iii) As noted in [11, Example 1], every (totally) mutually permutable product of two subgroups is a core-factorisation (see [3] for definitions).

(iv) By the initial paragraph, if $G$ is $\pi$-separable, then $G=H L$ is a core-factorisation for any $H \in \operatorname{Hall}_{\pi}(G)$ and $L \in \operatorname{Hall}_{\pi^{\prime}}(G)$.

Notice that, in the above statement (iv), the property that $H$ and $L$ have coprime orders is essential, as the next example shows.

Example 1. Let $G$ be a symmetric group of 4 letters. Then $G=A B$ where $A=\langle(1,3,2,4)$, $(1,2)(3,4)\rangle$ and $B=\langle(3,4),(2,3,4)\rangle$. Note that $G$ is clearly $\pi(A)$-separable (indeed it is soluble), but the unique minimal normal subgroup of $G$ is not covered by either $A$ or $B$, so $G=A B$ is not a core-factorisation.

We also provided a useful characterisation of core-factorisations via quotients (compare with [11, Lemma 2]).

Lemma 1. Let $1 \neq G=A B$ be the product of the subgroups $A$ and $B$. The following statements are pairwise equivalent:

(1) $G=A B$ is a core-factorisation.

(2) There exists a normal series $1=N_{0} \unlhd N_{1} \unlhd \cdots \unlhd N_{n-1} \unlhd N_{n}=G$ such that either $N_{i} / N_{i-1} \leqslant A N_{i-1} / N_{i-1}$ or $N_{i} / N_{i-1} \leqslant B N_{i-1} / N_{i-1}$, for each $1 \leq i \leq n$ (i.e. $N_{i} / N_{i-1}$ is covered by either $A$ or $\left.B\right)$.

(3) For every proper normal subgroup $K$ of $G$ it holds that there exists a normal subgroup $1 \neq M / K$ of $G / K$ such that either $M / K \leqslant A K / K$ or $M / K \leqslant B K / K$ (i.e. either $A$ or $B$ covers $M / K)$.

Further, in (2), each term $N_{i}$ of such (chief) normal series is prefactorised and $N_{i}=\left(N_{i} \cap\right.$ $A)\left(N_{i} \cap B\right)$ is also a core-factorisation.

If we adopt the bar convention in statement (3) for the quotients over $K$, we point out that this condition means $\operatorname{core}_{\bar{G}}(\bar{A}) \operatorname{core}_{\bar{G}}(\bar{B}) \neq 1$. This illustrates the given name for such factorisations.

Moreover, as noted in [11, Example 2], if $N$ is an arbitrary prefactorised normal subgroup of a core-factorisation $G=A B$, then $N=(N \cap A)(N \cap B)$ may not be a core-factorisation. Nevertheless, core-factorisations behave well with respect to quotients.

Lemma 2. [11, Lemma 1] Let $G=A B$ be a core-factorisation, and let $M$ be a proper normal subgroup of $G$. Then $G / M=(A M / M)(B M / M)$ is also a core-factorisation.

The next result on Hall $\pi$-subgroups of $\pi$-separable factorised groups is a key step for our development (indeed the $\pi$-separability condition can be relaxed to the $D_{\pi}$-property, as can be seen in [1, 1.3.2]).

Lemma 3. Let the $\pi$-separable group $G=A B$ be the product of the subgroups $A$ and $B$. Then there exists a Hall $\pi$-subgroup $H$ of $G$ such that $H=(H \cap A)(H \cap B)$, with $H \cap A$ a Hall $\pi$-subgroup of $A$ and $H \cap B$ a Hall $\pi$-subgroup of $B$.

In particular, if $G=A B$ is a core-factorisation, then $H=(H \cap A)(H \cap B)$ is also a core-factorisation. 
Proof. The first assertion is just a reformulation of [1, 1.3.2], so we concentrate on the second claim. We assume that $G=A B$ is a core-factorisation, and let us prove that $H=(H \cap A)(H \cap$ $B)$ so is. There exists a chief series $1=N_{0} \unlhd N_{1} \unlhd \cdots \unlhd N_{n-1} \unlhd N_{n}=G$ such that each $N_{i} / N_{i-1}$ is covered by either $A$ or $B$, for all $1 \leq i \leq n$.

Note that $1=N_{0} \cap H \unlhd N_{1} \cap H \unlhd \cdots \unlhd N_{n-1} \cap H \unlhd N_{n} \cap H=H$ is a normal series of $H$. We claim that each $\left(N_{i} \cap H\right) /\left(N_{i-1} \cap H\right)$ is covered by either $H \cap A$ or $H \cap B$, in order to apply Lemma 1. Since $G$ is $\pi$-separable, then $N_{i} / N_{i-1}$ is either a $\pi$-group or a $\pi^{\prime}$-group. In the latter case, $\left(N_{i} \cap H\right) /\left(N_{i-1} \cap H\right)=1$ is clearly covered by either $H \cap A$ or $H \cap B$. If $N_{i} / N_{i-1}$ is a $\pi$-group, since we may assume for instance that $N_{i} / N_{i-1}$ is covered by $A$, then $N_{i} / N_{i-1} \leqslant(H \cap A) N_{i-1} / N_{i-1}$ because $H \cap A \in \operatorname{Hall}_{\pi}(A)$. Now $N_{i}=N_{i-1}\left(N_{i} \cap H \cap A\right)$ and $H \cap N_{i}=H \cap N_{i-1}\left(N_{i} \cap H \cap A\right)=\left(N_{i} \cap H \cap A\right)\left(H \cap N_{i-1}\right) \leqslant(H \cap A)\left(H \cap N_{i-1}\right)$. Thus $\left(H \cap N_{i}\right) /\left(H \cap N_{i-1}\right)$ is covered by $H \cap A$ and we are done.

\section{On conjugacy class sizes of prime power order $\pi$-elements}

We start by presenting some preliminary results. The next elementary properties are used frequently and without further reference.

Lemma 4. Let $N$ be a normal subgroup of a group $G$, and $A$ be a subgroup of $G$. We have:

(a) $\left|x^{N}\right|$ divides $\left|x^{G}\right|$, for any $x \in N$.

(b) $\left|(x N)^{G / N}\right|$ divides $\left|x^{G}\right|$, for any $x \in G$.

(c) If $x N$ is a $\pi$-element of $A N / N$, then $x N=x_{1} N$ for some $\pi$-element $x_{1} \in A$.

The next observation is crucial in the sequel.

Remark 2. Let $G=A B$ be a $\pi$-separable group. Consider by Lemma 3 a Hall $\pi$-subgroup $H=(H \cap A)(H \cap B)$ of $G$ such that $H \cap A \in \operatorname{Hall}_{\pi}(A)$ and $H \cap B \in \operatorname{Hall}_{\pi}(B)$. Then, imposing arithmetical conditions on the class sizes of the (prime power order) $\pi$-elements in $A \cup B$ is equivalent to impose them on the class sizes of the (prime power order) elements in $(H \cap A) \cup(H \cap B)$, because of the conjugacy of Hall $\pi$-subgroups.

The lemma below is a transcription of a well-known Wielandt's result for a set of primes $\pi$.

Lemma 5. [6. Lemma 1] Let $G$ be a group and $H \in \operatorname{Hall}_{\pi}(G)$. If $\left|x^{G}\right|$ is a $\pi$-number for some $x \in H$, then $x \in \mathbf{O}_{\pi}(G)$.

Indeed, we can provide a useful $\pi$-separability criterion for factorised groups having a Hall $\pi$-subgroup by means of the previous class size condition.

Lemma 6. Assume that $G=A B$ with $\operatorname{Hall}_{\pi}(G) \neq \emptyset$. If $\left|x^{G}\right|$ is a $\pi$-number for every $\pi$-element $x \in A \cup B$ of prime power order, then $\mathrm{O}_{\pi}(G) \in \operatorname{Hall}_{\pi}(G)$. In particular, $G$ is $\pi$-separable.

Proof. Let $H \in \operatorname{Hall}_{\pi}(G)$. We choose $P=(P \cap A)(P \cap B) \in \operatorname{Syl}_{p}(G)$ for some $p \in \pi$. Clearly $P$ is $G$-conjugate to a Sylow $p$-subgroup of $H$, say $P_{1}$. Hence for some $g \in G$ we have $P=P_{1}^{g} \leqslant H^{g} \in \operatorname{Hall}_{\pi}(G)$. It follows by Lemma 5 that if $x \in(P \cap A) \cup(P \cap B)$, then $x \in \mathbf{O}_{\pi}(G)$, so $(P \cap A) \cup(P \cap B) \subseteq \mathbf{O}_{\pi}(G)$. Thus $P^{h} \leqslant \mathbf{O}_{\pi}(G)$ for all $h \in G$. Since this is valid for all $p \in \pi$, we deduce that $H=\mathbf{O}_{\pi}(G)$. The second claim follows directly.

In the next theorem, we get further information when the group has a core-factorisation. Our proof involves the following lemma, which makes use of the knowledge on the automorphism groups of the non-abelian simple groups. 
Lemma 7. [9. Lemma 2.6] Let $S$ be a simple group. Then there exists $r \in \pi(S)$ such that $\operatorname{gcd}\left(r,\left|\mathbf{C}_{S}(\alpha)\right|\right)=1$ for every non-trivial $\alpha \in \operatorname{Aut}(S)$ of order coprime to $|S|$.

Theorem A. Let $G=A B$ be a core-factorisation such that $\operatorname{Hall}_{\pi}(G) \neq \emptyset$. Then:

(1) Each $\left|x^{G}\right|$ is a $\pi$-number for every $\pi$-element $x \in A \cup B$ of prime power order if and only if $G$ is $\pi$-decomposable.

(2) Each $\left|x^{G}\right|$ is a $\pi$-number for every prime power order element $x \in A \cup B$ if and only if $G$ is $\pi$-decomposable and its Hall $\pi^{\prime}$-subgroup is abelian.

Proof. (1) The sufficiency of the condition is clear. Let us prove that $G=A B$ is $\pi$-decomposable whenever every $\left|x^{G}\right|$ is a $\pi$-number for each $\pi$-element $x \in A \cup B$ of prime power order. Take $G$ a minimal counterexample to the assertion. In virtue of Lemma 6 we can affirm that $H:=\mathbf{O}_{\pi}(G) \in \operatorname{Hall}_{\pi}(G)$, so $G$ is $\pi$-separable. Applying Lemma 3, we can choose $F \in$ $\operatorname{Hall}_{\pi^{\prime}}(G)$ prefactorised. Take $y \in F \cap A$. We claim that $G_{y}:=H\langle y\rangle$ satisfies the hypotheses of the theorem. We have

$$
G_{y}=\langle y\rangle(H \cap A)(H \cap B) \subseteq\left(G_{y} \cap A\right)\left(G_{y} \cap B\right) \subseteq G_{y},
$$

so $G_{y}$ is prefactorised and $\operatorname{Hall}_{\pi}\left(G_{y}\right)=\{H\} \neq \emptyset$. Now we take $p \in \pi$ and $P$ a prefactorised Sylow $p$-subgroup of $G_{y}$ as in Lemma 3 . Any $\left|x^{G}\right|$ is a $\pi$-number for the elements $x \in P \cap$ $G_{y} \cap A=P \cap A$. Hence, there exists $g \in G$ such that $F^{g} \leqslant \mathbf{C}_{G}(x)$. We can assume $g \in H$ because $G=H F$. Since $\langle y\rangle \leqslant F$, we get $\langle y\rangle^{g} \leqslant \mathbf{C}_{G_{y}}(x)$, so $\left|x^{G_{y}}\right|$ is a $\pi$-number. This is analogously valid for the elements in $P \cap G_{y} \cap B=P \cap B$, and for all $p \in \pi$. Now Remark 2 provides that all $\pi$-elements in $\left(G_{y} \cap A\right) \cup\left(G_{y} \cap B\right)$ of prime power order have conjugacy class size in $G_{y}$ a $\pi$-number. It remains to show that $G_{y}=\left(G_{y} \cap A\right)\left(G_{y} \cap B\right)$ is a core-factorisation. If we reproduce the techniques in the proof of Lemma 3 , we get that $1=N_{0} \cap H \unlhd N_{1} \cap H \unlhd \cdots \unlhd N_{n-1} \cap H \unlhd N_{n} \cap H=H$ is a normal series of $H$ such that each $\left(N_{i} \cap H\right) /\left(N_{i-1} \cap H\right)$ is covered by either $H \cap A \leqslant G_{y} \cap A$ or $H \cap B \leqslant G_{y} \cap B$. But $H$ and all the $N_{i}$ are normal in $G$, so $1=N_{0} \cap H \unlhd N_{1} \cap H \unlhd \cdots \unlhd N_{n-1} \cap H \unlhd N_{n} \cap H=H \unlhd H\langle y\rangle=G_{y}$ is a normal series of $G_{y}$. Moreover, $G_{y} / H$ is clearly covered by $G_{y} \cap A$ because $y \in G_{y} \cap A$. Thus all the factors are covered by either $G_{y} \cap A$ or $G_{y} \cap B$ and $G_{y}=\left(G_{y} \cap A\right)\left(G_{y} \cap B\right)$ is a core-factorisation by Lemma 1 .

If $G_{y}<G$, then it follows by minimality that $H \leqslant \mathbf{C}_{G}(y)$. Hence, we can suppose for some $y \in(F \cap A) \cup(F \cap B)$ that $G=H\langle y\rangle$; otherwise $H \leqslant \mathbf{C}_{G}(F)$, a contradiction. Indeed, by the decomposition of $y$ as product of prime power order elements, the same arguments apply and we can assume that $\mathrm{o}(y)$ is a $q$-number for some prime number $q \in \pi^{\prime}$.

Since the hypotheses are inherited by quotients of $G$, and the class of $\pi$-decomposable groups is a saturated formation, we may assume $\boldsymbol{\Phi}(G)=1$ and that there exists a unique minimal normal subgroup $N$ of $G$, so $N \leqslant H$. Thus $\mathbf{O}_{\pi^{\prime}}(G)=1$. As $G / N$ is $\pi$-decomposable, then $\langle y\rangle N \unlhd G$, and $[H,\langle y\rangle]=[H, y] \leqslant N$. Moreover, by coprime action we get $H=$ $[H, y] \mathbf{C}_{H}(y) \leqslant N \mathbf{C}_{H}(y)$, so $G=H\langle y\rangle=N \mathbf{C}_{G}(y)$.

We claim that $G=N\langle y\rangle$. Set $T:=N\langle y\rangle$ and assume that $T<G$. Note that $\operatorname{Hall}_{\pi}(T)=$ $\{N\} \neq \emptyset$. Since $G=A B$ is a core-factorisation and $N$ is the unique minimal normal subgroup of $G$, we may suppose that $N \leqslant A$. As $y \in(F \cap A) \cup(F \cap B)$, then clearly $T=(T \cap A)(T \cap B)$. If we consider the normal series $1 \unlhd N \unlhd N\langle y\rangle=T$, then the factors are covered by either $T \cap A$ or $T \cap B$ and $T=(T \cap A)(T \cap B)$ is a core-factorisation by Lemma 1 . Moreover, the class size conditions are inherited by $T$ because it is prefactorised and normal in $G$. By minimality we obtain that $N \leqslant \mathbf{C}_{G}(y)$ and $G=N \mathbf{C}_{G}(y)=\mathbf{C}_{G}(y)$, which leads to a contradiction. Therefore, $G=N\langle y\rangle$.

Next we demonstrate that $N$ is non-abelian. Otherwise $N=\mathbf{C}_{G}(N)$ because of standard group theoretic arguments ([7, Theorem A - 10.6]). By coprime action we get $N=[N, y] \times$ 
$\mathbf{C}_{N}(y)$. But $\mathbf{C}_{N}(y)$ is normal in $G$ and $N=\mathbf{C}_{G}(N)$, so necessarily $\mathbf{C}_{N}(y)=1$. Since $N$ is $t$-elementary abelian for some prime $t \in \pi$, any non-trivial element $n \in N \leqslant A$ satisfies that $\left|n^{G}\right|$ is a $\pi$-number, so a $G$-conjugate of $n$ lies in $\mathbf{C}_{N}(y)=1$, a contradiction.

Thus $N$ is non-abelian and we can write $N=L_{1} \times \cdots \times L_{k}$ where all $L_{i}$ are isomorphic nonabelian simple groups and they form a full $G$-conjugacy class of subgroups. Since $G=N\langle y\rangle$, then $\langle y\rangle$ acts transitively on $\left\{L_{1}, \ldots, L_{k}\right\}$. If $k>1$, then $k=\left|G: \mathbf{N}_{G}\left(L_{1}\right)\right|$ divides the order of the $q$-element $y$, so $k$ is a non-trivial $q$-power. Now for any $1 \neq x_{1} \in L_{1}$ of prime power order we get that $\mathbf{C}_{G}\left(x_{1}\right) \leqslant \mathbf{N}_{G}\left(L_{1}\right)$, so $k$ divides $\left|x_{1}^{G}\right|$. But this class size is a $\pi$-number because $x_{1} \in N \leqslant A$ is a prime power order $\pi$-element. It follows that $k=1$ and $N$ is simple. Now we can apply Lemma 7 in order to affirm that there exists a prime $s \in \pi(N)$ such that $s$ does not divide $\left|\mathbf{C}_{N}(y)\right|$. Let $x$ be a non-trivial $s$-element in $N \leqslant A$. Since by hypotheses there is a conjugate of $x$ which lies in $\mathbf{C}_{N}(y)=1$, we have reached the final contradiction. The proof of (1) is now completed.

(2) It is enough to show the necessity of the condition. Assume that $\left|x^{G}\right|$ is a $\pi$-number for every prime power order element $x \in A \cup B$. Clearly, $G$ is $\pi$-decomposable by (1). Moreover, its unique Hall $\pi^{\prime}$-subgroup $\mathbf{O}_{\pi^{\prime}}(G)$ is prefactorised by Lemma 3 . Since $\mathbf{O}_{\pi^{\prime}}(G) \cap A$ and $\mathbf{O}_{\pi^{\prime}}(G) \cap$ $B$ are generated by prime power order elements, all of which lying in $\mathbf{Z}\left(\mathbf{O}_{\pi^{\prime}}(G)\right)$ due to the class size assumptions, then $\mathbf{O}_{\pi^{\prime}}(G)$ is abelian.

A question which remains open is whether the hypothesis of being a core-factorisation in Theorem A can be eliminated. Moreover, when we consider the trivial factorisation $G=A=B$ in the above theorem, we retrieve the next result in [14]. In fact, our arguments provide an alternative proof. We remark that the proof given in that paper uses deeply the CFSG via a result due to Fein, Kantor and Schacher (see [14, Lemma 2]).

Corollary 1. 14, Theorem 3.1] Let $G$ be a group with $\operatorname{Hall}_{\pi}(G) \neq \emptyset$. Then each $\left|x^{G}\right|$ is a $\pi$-number for every $\pi$-element $x \in G$ of prime power order if and only if $G$ is $\pi$-decomposable.

Remark 3. Actually, when all the $\pi$-elements are considered in the above result (not only those of prime power order), then the CFSG can be avoided (see either [6. Supplement to Theorem 1] or Lemma 9 below).

Zhao et al. also provided in [14, Theorem 3.2] a similar characterisation to the one in Theorem A but considering a factorised group $G=A B$ with one factor which is subnormal. It is worth to remark that, if $A$ is subnormal, then for every element $x \in A$ it holds that $\left|x^{A}\right|$ divides $\left|x^{G}\right|$, although in general this is not the case. Besides, there exists a normal subgroup of $G$ which contains $A$, so this normal subgroup is prefactorised.

Example 2. Notice that, a priori, groups with a core-factorisation and factorised groups with one subnormal factor are different types of groups. For instance, let $G$ be the natural wreath product of a symmetric group of degree 3 and a cyclic group $\langle z\rangle$ of order 2 . If $A=\left\langle(2,3),(1,2,3)^{z}\right.$, $\left.(2,3)^{z}\right\rangle$ and $B=\left\langle(1,3,2)(4,5,6)^{z},(1,3,2)(4,5,6)^{z} z\right\rangle$, then $G=A B$ is not a core-factorisation and $B$ is subnormal in $G$. On the other hand, it is not difficult to find core-factorisations where the factors are neither subnormal in the whole group nor mutually permutable ([11 Example 2]).

Next, we deal with the dual condition on the class sizes of prime power order $\pi$-elements, i.e. when they are not divisible by any prime in $\pi$. We characterise arbitrary factorisations of $\pi$-separable groups which have abelian Hall $\pi$-subgroups through elementary reasonaments.

Proposition 1. Let $G=A B$ be a $\pi$-separable group. Then $\left|x^{G}\right|$ is a $\pi^{\prime}$-number for each $\pi$ element $x \in A \cup B$ of prime power order if and only if the Hall $\pi$-subgroups of $G$ are abelian. Moreover, if this occurs, then the $\pi$-length of $G$ is at most 1. 
Proof. We can work with $H=(H \cap A)(H \cap B) \in \operatorname{Hall}_{\pi}(G)$ such that $H \cap A \in \operatorname{Hall}_{\pi}(A)$ and $H \cap B \in \operatorname{Hall}_{\pi}(B)$ in virtue of Lemma 3 . The converse of the first claim is clear by Remark 2. So let us prove that $H=(H \cap A)(H \cap B)$ is abelian when $\left|x^{G}\right|$ is a $\pi^{\prime}$-number for each $\pi$-element $x \in A \cup B$ of prime power order. Suppose that the assertion is false and let us take $G$ a minimal counterexample. Then $\mathbf{O}_{\pi^{\prime}}(G)=1$ by minimality, and so $\mathbf{C}_{G}\left(\mathbf{O}_{\pi}(G)\right) \leqslant$ $\mathrm{O}_{\pi}(G)$. Take a Sylow $q$-subgroup $Q$ of $H \cap A$. Then each $y \in Q$ satisfies by assumption that $y \in \mathbf{C}_{G}\left(\mathbf{O}_{\pi}(G)\right) \leqslant \mathbf{O}_{\pi}(G)$. Since $H \cap A$ is generated by its Sylow subgroups, it follows that $H \cap A \leqslant \mathbf{C}_{G}\left(\mathbf{O}_{\pi}(G)\right) \leqslant \mathbf{O}_{\pi}(G)$ and analogously for $H \cap B$. Hence $H \leqslant \mathbf{C}_{G}\left(\mathbf{O}_{\pi}(G)\right) \leqslant$ $\mathbf{O}_{\pi}(G)$, so $H$ is abelian. The last claim follows easily from the fact that $\mathbf{O}_{\pi}\left(G / \mathbf{O}_{\pi^{\prime}}(G)\right)$ is self-centralising in $G / \mathbf{O}_{\pi^{\prime}}(G)$.

Example 3. Without the $\pi$-separability hypothesis, the previous result is not true, even for a not necessarily factorised group: Let $G=J_{4}$ be a Janko group, and let $\pi=\{3\}$. Then all the 3 -elements of $G$ have conjugacy class size not divisible by 3, although a Sylow 3-subgroup is non-abelian. This example appears in [13].

Now we prove a result related to the above theorems.

Proposition 2. Let $G=A B$ be a $\pi$-separable group. Assume that a given prime $p$ does not divide $\left|x^{G}\right|$ for each $\pi$-element $x \in A \cup B$ of prime power order. Then there exists a Sylow p-subgroup of $G$ which normalises some Hall $\pi$-subgroup of $G$.

Proof. We may assume clearly that $p \in \pi^{\prime}$. Besides, by conjugation and Lemma 3 , we may work with $H=(H \cap A)(H \cap B) \in \operatorname{Hall}_{\pi}(G)$. Let $G$ be a counterexample of least possible order. If $\mathbf{O}_{\pi}(G) \neq 1$, then by minimality we get the thesis. Hence we necessarily have that $N:=\mathbf{O}_{\pi^{\prime}}(G) \neq 1$.

We claim that $p$ does not divide $\left|N: \mathbf{N}_{N}(H)\right|$. Certainly, we may suppose that $p \in \pi(N)$. Let $P_{0} \in \operatorname{Syl}_{p}(N)$, and $G=\mathbf{N}_{G}\left(P_{0}\right) N$ in virtue of Frattini's argument. For $q \in \pi$, let $Q=(Q \cap H \cap A)(Q \cap H \cap B)=(Q \cap A)(Q \cap B)$ be a prefactorised Sylow $q$-subgroup of $H$. If $a \in Q \cap A$, then by hypotheses we get $a \in \mathbf{C}_{Q \cap A}\left(P_{0}^{n}\right)$ for some $n \in N$. It follows

$$
(Q \cap A) N \subseteq \mathbf{C}_{(Q \cap A) N}\left(P_{0}\right) N \subseteq(Q \cap A) N,
$$

so $(Q \cap A) N=\mathbf{C}_{(Q \cap A) N}\left(P_{0}\right) N \leqslant \mathbf{C}_{Q N}\left(P_{0}\right) N$. We can argue analogously with $Q \cap B$ and thus $Q N=(Q \cap A)(Q \cap B) N=\mathbf{C}_{Q N}\left(P_{0}\right) N \leqslant \mathbf{C}_{H N}\left(P_{0}\right) N \leqslant H N$. Now for any $h \in H$, we also have $Q^{h} N=(Q N)^{h} \leqslant\left(\mathbf{C}_{H N}\left(P_{0}\right) N\right)^{h}=\mathbf{C}_{H N}\left(P_{0}^{h}\right) N$. But $h \in G=\mathbf{N}_{G}\left(P_{0}\right) N$, so we may assume $h \in N$ and so $Q^{h} N \leqslant \mathbf{C}_{H N}\left(P_{0}^{h}\right) N=\left(\mathbf{C}_{H N}\left(P_{0}\right) N\right)^{h}=\mathbf{C}_{H N}\left(P_{0}\right) N$. Since this is valid for each $q \in \pi$ we deduce $H N=\mathbf{C}_{H N}\left(P_{0}\right) N$. But $N$ is a $\pi^{\prime}$-group, so there exists $n \in N$ such that $H \leqslant \mathbf{C}_{H N}\left(P_{0}^{n}\right)$. Hence $P_{0}^{n} \leqslant \mathbf{C}_{N}(H) \leqslant \mathbf{N}_{N}(H) \leqslant N$. As $P_{0} \in \operatorname{Syl}_{p}(N)$, it follows that $p$ does not divide $\left|N: \mathbf{N}_{N}(H)\right|$.

On the other hand, by minimality there exists a Sylow $p$-subgroup $P$ of $\mathrm{G}$ such that $P \leqslant$ $\mathbf{N}_{G}(H N)$. Again Frattini's argument for Hall $\pi$-subgroups produces $N H P=N H \mathbf{N}_{N H P}(H)$ $=N \mathbf{N}_{N H P}(H)$. Therefore $p$ does not divides $\left|N H P: \mathbf{N}_{N H P}(H)\right|=\left|N: \mathbf{N}_{N}(H)\right|$. Thus there is a Sylow $p$-subgroup of $H N P$ (which is a Sylow $p$-subgroup of $G$ ) that normalises $H$.

In particular, when $G=A=B$ and $\pi=\{q\}$ we partially get [5, Theorem 4.1]. It is worth to remark again that both Propositions 1 and 2 hold for any arbitrary factorisation of a $\pi$-separable group $G=A B$.

\section{On conjugacy class sizes of $\pi$-elements}

The assumptions in Corollary 1 imply that the elements in the centre of a Hall $\pi$-subgroup $H$ of a group $G$ have to be central in $G$. Thus, a more general approach is to consider only 
the elements in $H \backslash \mathbf{Z}(H)$, as Berkovich and Kazarin did through elementary arguments in [6. Supplement to Theorem 1] for $\pi$-separable groups. For the sake of completeness, we present a proof of that result for groups which have a Hall $\pi$-subgroup (see Lemma 9 below).

Lemma 8. Let $H$ be a proper subgroup of a group $G$. Then $G=\langle G \backslash H\rangle$.

Proof. This follows from the fact that $G=H \cup\langle G \backslash H\rangle$.

Lemma 9. Let $G$ be a group with a non-abelian Hall $\pi$-subgroup $H$. Then $G$ is $\pi$-decomposable whenever every class size of elements in $H \backslash \mathbf{Z}(H)$ is a $\pi$-number.

Proof. In virtue of Lemma 5 it follows that every element $x \in H \backslash \mathbf{Z}(H)$ lies in $\mathbf{O}_{\pi}(G)$, and Lemma 8 leads to $H=\langle H \backslash \mathbf{Z}(H)\rangle \leqslant \mathbf{O}_{\pi}(G)$. So $G$ has a normal Hall $\pi$-subgroup and it is $\pi$-separable. Let $F$ be a Hall $\pi^{\prime}$-subgroup of $G$. If $g \in H \backslash \mathbf{Z}(H)$, then by hypotheses $g \in \mathbf{C}_{H}\left(F^{x}\right)$ for some $x \in H$ since $G=H F$. Thus $H \subseteq \cup_{x \in H}\left(\mathbf{Z}(H) \mathbf{C}_{H}(F)\right)^{x} \subseteq H$, so $H=\mathbf{Z}(H) \mathbf{C}_{H}(F)$ and $\mathbf{C}_{H}(F)$ is normal in $H$. Thus, every element $g \in H \backslash \mathbf{Z}(H)$ lies in $\mathbf{C}_{H}(F)$. Since $H=\langle H \backslash \mathbf{Z}(H)\rangle \leqslant \mathbf{C}_{H}(F)$, it follows $G=H F=H \times F$, as desired.

Example 4. In view of the previous section, one might wonder whether the hypotheses in Lemma 9 can be restricted to only prime power order $\pi$-elements. However, this is simply not possible:

Let $G$ be the direct product of a symmetric group of degree 3 and a non-abelian group of order 55, and let $\pi=\{2,3,11\}$. Then $H \in \operatorname{Hall}_{\pi}(G)$ is clearly non-abelian, $G$ is not $\pi$ decomposable, and $\left|x^{G}\right|$ is a $\pi$-number for every element $x \in H \backslash \mathbf{Z}(H)$ of prime power order.

Our next objective is to generalise Lemma 9 to $\pi$-separable groups which possess a corefactorisation.

Theorem 1. Let $G=A B$ be a core-factorisation, and suppose that $G$ is $\pi$-separable. Let $H=(H \cap A)(H \cap B)$ be a Hall $\pi$-subgroup of $G$ such that $H \cap X \in \operatorname{Hall}_{\pi}(X)$ for $X \in\{A, B\}$, and assume that $H$ is non-abelian. Then the following statements are equivalent:

(1) Every element in $((H \cap A) \cup(H \cap B)) \backslash \mathbf{Z}(H)$ has $G$-class size a $\pi$-number.

(2) For each $X \in\{A, B\}$, either $H \cap X \leqslant \mathbf{Z}(H)$ or $H \cap X \leqslant \mathbf{C}_{H}(F)$ for every $F \in$ $\operatorname{Hall}_{\pi^{\prime}}(G)$.

In case (2), if $H \cap X \leqslant \mathbf{Z}(H)$, then $X$ has $\pi$-length at most 1 ; and if $H \cap X \leqslant \mathbf{C}_{H}(F)$ for every $F \in \operatorname{Hall}_{\pi^{\prime}}(G)$, then $X$ is $\pi$-decomposable.

Proof. Let $F=(F \cap A)(F \cap B)$ be a prefactorised Hall $\pi^{\prime}$-subgroup of $G$ as in Lemma 3 . Since $H \cap X \in \operatorname{Hall}_{\pi}(X)$, the last claim of the result follows from the fact that either $H \cap X$ is abelian, or $H \cap X \leqslant \mathbf{C}_{H \cap X}(F) \leqslant \mathbf{C}_{X}(F \cap X)$ being $F \cap X \in$ Hall $_{\pi^{\prime}}(X)$. Moreover, the implication (2) $\Rightarrow(1)$ is clear. Therefore, it is enough to show that $(1) \Rightarrow(2)$. Notice that $H$ is non-abelian by assumption, so there exists some $X \in\{A, B\}$ such that $H \cap X \nless \mathbf{Z}(H)$. Now, let us fix some arbitrary $F \in \operatorname{Hall}_{\pi^{\prime}}(G)$, and note that $G=H F$. We split the proof in a number of steps.

STEP 1: If $H \cap X \nless \mathbf{Z}(H)$ and $H \cap X$ is normal in $H$, then $H \cap X \leqslant \mathbf{C}_{H}(F)$.

Let $\{X, Y\}=\{A, B\}$. We claim that $H=(H \cap X) \mathbf{C}_{H}(F) \mathbf{Z}(H)$, and we distinguish two cases. If $H \cap Y \leqslant \mathbf{Z}(H)$, then clearly $H=(H \cap X) \mathbf{Z}(H)=(H \cap X) \mathbf{C}_{H}(F) \mathbf{Z}(H)$. If $H \cap Y \nless \mathbf{Z}(H)$, then we can pick $y \in(H \cap Y) \backslash \mathbf{Z}(H)$. By our hypotheses, it follows that $y \in \mathbf{C}_{G}(F)^{h}$ for some $h \in H$, and hence $H \cap Y \subseteq \cup_{h \in H} \mathbf{C}_{H}(F)^{h} \mathbf{Z}(H)$. Since $H \cap X \unlhd H$, then

$$
H \subseteq(H \cap X) \bigcup_{h \in H} \mathbf{C}_{H}(F)^{h} \mathbf{Z}(H) \subseteq \bigcup_{h \in H}\left[(H \cap X) \mathbf{C}_{H}(F) \mathbf{Z}(H)\right]^{h} \subseteq H .
$$


This fact yields $H=(H \cap X) \mathbf{C}_{H}(F) \mathbf{Z}(H)$.

Now we choose $x \in(H \cap X) \backslash \mathbf{Z}(H)$. Thus, we get $x \in \mathbf{C}_{H \cap X}\left(F^{h}\right)$ with $h \in(H \cap X) \mathbf{Z}(H)$. Indeed, $h=g z$ with $g \in H \cap X$ and $z \in \mathbf{Z}(H)$, so $x^{g^{-1}}=x^{h^{-1}} \in \mathbf{C}_{H \cap X}(F)$. We deduce

$$
H \cap X=\bigcup_{g \in H \cap X} \mathbf{C}_{H \cap X}(F)^{g}(\mathbf{Z}(H) \cap X)=\bigcup_{g \in H \cap X}\left[\mathbf{C}_{H \cap X}(F)(\mathbf{Z}(H) \cap X)\right]^{g},
$$

so $H \cap X=\mathbf{C}_{H \cap X}(F)(\mathbf{Z}(H) \cap X)$ and $\mathbf{C}_{H \cap X}(F)$ is normal in $H \cap X$. Now each element in $(H \cap X) \backslash \mathbf{Z}(H)$ lies in $\mathbf{C}_{H \cap X}(F)$. Since $(H \cap X) \backslash \mathbf{Z}(H)=(H \cap X) \backslash(\mathbf{Z}(H) \cap X)$, in virtue of Lemma 8 we obtain $H \cap X=\langle(H \cap X) \backslash \mathbf{Z}(H)\rangle \leqslant \mathbf{C}_{H}(F)$, as wanted.

Now we assume without loss of generality that $H \cap A \nless \mathbf{Z}(H)$. So the remainder of the proof aims to show that $H \cap A \leqslant \mathbf{C}_{H}(F)$.

STEP 2: We may suppose that neither $H \cap A$ nor $H \cap B$ are normal in $H$.

By Step 1, we may assume that $H \cap A$ is not normal in $H$. If $H \cap B \leqslant \mathbf{Z}(H)$, then $H=(H \cap A) \mathbf{Z}(H)$ and $H \cap A \unlhd H$, a contradiction. Therefore $H \cap B \nless \mathbf{Z}(H)$. If $H \cap B$ is normal in $H$, then by Step 1 it centralises $F$. So $H=(H \cap A) \mathbf{C}_{H}(F)$ and arguing similarly as in the last paragraph of Step 1, we can deduce that $H \cap A \leqslant \mathbf{C}_{H}(F)$.

STEP 3: If $N$ is a minimal normal subgroup of $G$, then $N$ is a $\pi$-group.

Otherwise, we may assume that $N$ is a $\pi^{\prime}$-group because $G$ is $\pi$-separable. We argue by induction on the order of $G$. We claim that the quotient $\bar{G}:=G / N$ inherits the hypotheses. Clearly we can assume $1 \neq \bar{G}$, since $N=G$ implies the result trivially. Note that for $X \in$ $\{A, B\}$, it holds $\overline{H \cap X} \leqslant \bar{H} \cap \bar{X}$ and, as $H \cap X \in \operatorname{Hall}_{\pi}(X)$, then $\overline{H \cap X}=\bar{H} \cap \bar{X}$. Thus $\bar{H}$ is prefactorised as in Lemma 3 . Also $\bar{H}$ is non-abelian, $\bar{G}$ is a core-factorisation, and the class size condition is clearly inherited by quotients of $G$, so $\bar{G}$ satisfies the assumptions.

By induction either $\bar{H} \cap \bar{X} \leqslant \mathbf{Z}(\bar{H})$ or $\bar{H} \cap \bar{X} \leqslant \mathbf{C}_{\bar{G}}(\bar{F})$ for all $X \in\{A, B\}$. If $\bar{H} \cap \bar{X} \leqslant$ $\mathbf{Z}(\bar{H})$, then $[H \cap X, H] \leqslant N \cap H=1$ and $H \cap X \leqslant \mathbf{Z}(H)$, a contradiction with Step 2 . Therefore we necessarily have $[H \cap X, F] \leqslant N \leqslant F$, so $H \cap X$ normalises $F$. Since this is valid for all $X \in\{A, B\}$, we get that $F$ is normal in $G$. If $x \in(H \cap A) \backslash \mathbf{Z}(H)$, then the fact that $\left|x^{G}\right|$ is a $\pi$-number implies that $x \in \mathbf{C}_{H}(F)$. As $H \cap A$ is generated by the elements in $(H \cap A) \backslash \mathbf{Z}(H)$, then $H \cap A$ centralises $F$, as wanted.

\section{STEP 4: Conclusion.}

Since $G=A B$ is a core-factorisation, we can choose a minimal normal subgroup $N$ of $G$ which is covered by some $X \in\{A, B\}$. Moreover, $N$ is a $\pi$-group by the previous step. We consider $\bar{G}:=G / N$. If $\bar{H}$ is abelian, then $1 \neq H^{\prime} \leqslant N \leqslant H \cap X \leqslant H$, so $H \cap X$ is normal in $H$, which cannot happen because of Step 2 . Thus, $\bar{G}$ inherits the hypotheses, and so $\bar{G}$ satisfies the thesis by induction on $|G|$.

Now if $\overline{H \cap X} \leqslant \mathbf{Z}(\bar{H})$, then $[H \cap X, H \cap Y] \leqslant N \leqslant H \cap X$, so $H \cap X$ is normal in $H$, a contradiction again with Step 2. Therefore, both $\overline{H \cap A}$ and $\overline{H \cap B}$ centralise $\bar{F}$, and it follows that $\bar{H}$ centralises $\bar{F}$. Hence $F N$ normal in $G$, and for all $g \in G$ there is some $n \in N$ such that $F^{g}=F^{n}$.

Next we claim that $N=(\mathbf{Z}(H) \cap N) \mathbf{C}_{N}(F)$. If $N \leqslant \mathbf{Z}(H)$ then the claim is clear. If $N \nless \mathbf{Z}(H)$, then we can take $m \in N \backslash \mathbf{Z}(H)$ and by assumptions $m \in \mathbf{C}_{N}(F)^{n}$ for some $n \in N$. Hence $N=\cup_{n \in N}\left[(\mathbf{Z}(H) \cap N) \mathbf{C}_{N}(F)\right]^{n}$ and so $N=(\mathbf{Z}(H) \cap N) \mathbf{C}_{N}(F)$.

Consequently, since each element $x \in(H \cap A) \backslash \mathbf{Z}(H)$ lies in $\mathbf{C}_{H}(F)^{n}$ for some $n \in N$ and $N=(\mathbf{Z}(H) \cap N) \mathbf{C}_{N}(F)$, it follows $x \in \mathbf{C}_{H}(F)$. Thus $H \cap A=\langle(H \cap A) \backslash \mathbf{Z}(H)\rangle \leqslant \mathbf{C}_{H}(F)$.

Finally, we can argue analogously with $H \cap B$ in case that $H \cap B \nless \mathbf{Z}(H)$. The result is now proved. 
Example 5. In contrast to Lemma 9 , the following example shows that in Theorem 1 we cannot affirm that $G$ is $\pi$-decomposable: Let $A$ be a dihedral group of order 8 and let $B$ be a dihedral group of order 10, and consider $\pi=\{2\}$. Then $G=A \times B$ satisfies the hypotheses in Theorem 1 but clearly it is not 2-decomposable.

As a consequence, we obtain the next result.

Theorem B. Let $G=A B$ be a core-factorisation, and suppose that $G$ is $\pi$-separable. Let $H=(H \cap A)(H \cap B)$ be a Hall $\pi$-subgroup of $G$ such that $H \cap X \in \operatorname{Hall}_{\pi}(X)$ for all $X \in\{A, B\}$. Then the next assertions are pairwise equivalent:

(1) Every element in $(H \cap A) \cup(H \cap B)$ has $G$-class size either a $\pi$-number or a $\pi^{\prime}$-number.

(2) For each $X \in\{A, B\}$, either $H \cap X \leqslant \mathbf{C}_{H}(F)$ for every $F \in \operatorname{Hall}_{\pi^{\prime}}(G)$ or $H \cap X \leqslant$ $\mathbf{Z}(H)$.

In addition, for $X \in\{A, B\}$ :

(a) $H \cap X \leqslant \mathbf{Z}(H)$ if and only if all $\left|x^{G}\right|$ are $\pi^{\prime}$-numbers for $x \in H \cap X$. In this case the $\pi$-length of $X$ is at most 1.

(b) $H \cap X \leqslant \mathbf{C}_{H}(F)$ for every $F \in \operatorname{Hall}_{\pi^{\prime}}(G)$ if and only if all $\left|x^{G}\right|$ are $\pi$-numbers for $x \in H \cap X$. In this case $X$ is $\pi$-decomposable.

Proof. The implication (2) $\Rightarrow(1)$ is clear. Let us prove (1) $\Rightarrow(2)$. We may suppose that $H$ is non-abelian. We work by induction on the order of $G$, and we first claim that we can assume $\mathbf{O}_{\pi^{\prime}}(G) \neq 1$. Otherwise $\mathbf{O}_{\pi}(G)$ is self-centralising in $G$. If $x \in(H \cap A) \cup(H \cap B)$, then $\left|x^{G}\right|$ is either a $\pi$-number or a $\pi^{\prime}$-number. In the first case $x \in \mathbf{O}_{\pi}(G)$ because of Lemma 5, and in the second case $x \in \mathbf{C}_{G}\left(\mathbf{O}_{\pi}(G)\right) \leqslant \mathbf{O}_{\pi}(G)$. Since this is valid for every element $x \in(H \cap A) \cup(H \cap B)$, it follows that $\mathbf{O}_{\pi}(G)=H$ is prefactorised. Thus, for each $X \in\{A, B\}$ the elements $x \in \mathbf{O}_{\pi}(G) \cap X$ with $\left|x^{G}\right|$ a $\pi^{\prime}$-number lie in $\mathbf{Z}\left(\mathbf{O}_{\pi}(G)\right)$. So any $\left|x^{G}\right|$ is a $\pi$-number for the elements $x \in((H \cap A) \cup(H \cap B)) \backslash \mathbf{Z}(H)$. Applying Theorem 1 we obtain for each $X \in\{A, B\}$ that either $H \cap X \leqslant \mathbf{Z}(H)$ or $H \cap X=\mathbf{O}_{\pi}(G) \cap X \leqslant \mathbf{C}_{G}(F)$ for every $\pi^{\prime}$-Hall subgroup $F$ of $G$, as desired. It follows then $\mathbf{O}_{\pi^{\prime}}(G) \neq 1$.

Now, by induction, we get that $\bar{G}:=G / \mathrm{O}_{\pi^{\prime}}(G)$ satisfies the thesis. Let $X \in\{A, B\}$, so we have either $\overline{H \cap X}=\bar{H} \cap \bar{X} \leqslant \mathbf{Z}(\bar{H})$ or $\overline{H \cap X}=\bar{H} \cap \bar{X} \leqslant \mathbf{C}_{\bar{G}}(\bar{F})$ for any $F \in \operatorname{Hall}_{\pi^{\prime}}(G)$. The first case leads to $[H \cap X, H] \leqslant H \cap \mathbf{O}_{\pi^{\prime}}(G)=1$, so $H \cap X \leqslant \mathbf{Z}(H)$ and we are done. Hence, let us suppose $\overline{H \cap X} \leqslant \mathbf{C}_{\bar{G}}(\bar{F})$ and $H \cap X \nless \mathbf{Z}(H)$. Now if $\left|x^{G}\right|$ is a $\pi^{\prime}$-number for some $x \in(H \cap X) \backslash \mathbf{Z}(H)$, then $\left|\bar{x}^{\bar{G}}\right|$ so is too. But $\bar{x} \in \overline{H \cap X} \leqslant \mathbf{C}_{\bar{G}}(\bar{F})$, and we get that $\bar{x}$ is central in $\bar{G}$. In particular, $[H,\langle x\rangle] \leqslant \mathbf{O}_{\pi^{\prime}}(G) \cap H=1$, so $x \in \mathbf{Z}(H)$, a contradiction. Thus, any $\left|x^{G}\right|$ is a $\pi$-number for the elements $x \in((H \cap A) \cup(H \cap B)) \backslash \mathbf{Z}(H)$, so the thesis follows as an application again of Theorem 1. This completes the proof of $(1) \Rightarrow(2)$.

Next we prove (a). For the first claim, certainly only the sufficiency of the condition is in doubt. So let us suppose that all $\left|x^{G}\right|$ are $\pi^{\prime}$-numbers for $x \in H \cap X$. By (2), either $H \cap X \leqslant \mathbf{Z}(H)$ or $H \cap X \leqslant \mathbf{C}_{H}(F)$ for every $F \in \operatorname{Hall}_{\pi^{\prime}}(G)$. In the first case we are done, and in the second case it follows that any element in $H \cap X$ is central in $G$, so $H \cap X \leqslant \mathbf{Z}(H)$ also. Moreover, the last assertion follows from the fact that $X$ has abelian Hall $\pi$-subgroups.

Finally we prove (b). Again, it is enough to show in the first claim the sufficiency of the condition. Let us suppose that all $\left|x^{G}\right|$ are $\pi$-numbers for $x \in H \cap X$. If the case $H \cap X \leqslant \mathbf{Z}(H)$ in (2) holds, then $H \cap X$ is central in $G$ and we are done. So $H \cap X \leqslant \mathbf{C}_{H}(F)$ for every $F \in \operatorname{Hall}_{\pi^{\prime}}(G)$. Further, the last assertion can be deduced from the fact that $H \cap X$ centralises a prefactorised Hall $\pi^{\prime}$-subgroup $F$ of $G$ as in Lemma 3, so $H \cap X \leqslant \mathbf{C}_{X}(F \cap X)$ where $F \cap X \in \operatorname{Hall}_{\pi^{\prime}}(X)$. 
Example 6. The $\pi$-separability assumption in the previous result is necessary: Let $G=A \times B$ be the direct product of $A=J_{4}$ a Janko group and $B=C_{3}$ a cyclic group of order 3, and let $\pi=\{3\}$. Note that this is clearly a core-factorisation, and $G$ is not 3-separable. Moreover, if we take $P \in \operatorname{Syl}_{3}(G)$ such that $P=(P \cap A)(P \cap B)$ with $P \cap A \in \operatorname{Syl}_{3}(A)$ and $B=$ $P \cap B \in \operatorname{Syl}_{3}(B)$, then $P$ is non-abelian and all $\left|x^{G}\right|$ are not divisible by 3 for the elements $x \in((P \cap A) \cup(P \cap B)) \backslash \mathbf{Z}(P)=(P \cap A) \backslash \mathbf{Z}(P)$. However, neither $P \cap A$ is central in $P$ nor $P \cap A$ centralises every Hall $3^{\prime}$-subgroup of $G$.

When $G=A=B$ in Theorem $\mathrm{B}$, the corollary below follows.

Corollary C. Let $G$ be a $\pi$-separable group. Then the following statements are pairwise equivalent:

(1) Each $\left|x^{G}\right|$ is either a $\pi$-number or a $\pi^{\prime}$-number for every $\pi$-element $x \in G$.

(2) Either $G$ is $\pi$-decomposable or it has abelian Hall $\pi$-subgroups and its $\pi$-length is at most 1 .

(3) For every $\pi$-element $x \in G$, either all $\left|x^{G}\right|$ are $\pi$-numbers or they are all $\pi^{\prime}$-numbers.

In [8, Theorem 4] (see the next theorem, which is a little reformulation) Dolfi characterised the so-called class- $\pi$-separable groups, i.e. groups all of whose class sizes are either $\pi$-numbers or $\pi^{\prime}$-numbers.

Theorem 2. A group $G$ is class- $\pi$-separable if and only if, up to abelian direct factors, one of the following two cases happens:

(1) $G$ is either a $\pi$-group or a $\pi^{\prime}$-group.

(2) Up to interchanging $\pi$ and $\pi^{\prime}, G=H L$ with $H \in \operatorname{Hall}_{\pi}(G), L \in \operatorname{Hall}_{\pi^{\prime}}(G), L \unlhd G$, both $H$ and $L$ are abelian, and $G / \mathbf{O}_{\pi}(G)$ is a Frobenius group. Indeed, $\mathbf{O}_{\pi}(G)=\mathbf{Z}(G)$, the set of the class sizes of $G$ is $\left\{1,\left|H / \mathbf{O}_{\pi}(G)\right|,|L|\right\}$, and $G$ is soluble.

Motivated by Dolfi's result, we introduce the following factorised-group version of the concept of class- $\pi$-separability.

Definition 2. Let $G=A B$ be the product of two subgroups $A$ and $B$. We say that $G=A B$ is a class- $\pi$-separable factorisation whenever $\left|x^{G}\right|$ is either a $\pi$-number or a $\pi^{\prime}$-number for every element $x \in A \cup B$.

Certainly, $G=A B$ is a class- $\pi$-separable factorisation if and only if it is a class- $\pi^{\prime}$-separable factorisation. Besides, any central product of two class- $\pi$-separable groups provides a class- $\pi$ separable factorisation.

We cannot assert in a class- $\pi$-separable factorisation $G=A B$, a priori, that both $A$ and $B$ are class- $\pi$-separable groups. This is because, for $x \in A$, there is no relation in general between the sets $\pi\left(\left|x^{A}\right|\right)$ and $\pi\left(\left|x^{G}\right|\right)$. Nevertheless, under the additional assumption of being a corefactorisation, we determine in Theorem $\mathrm{D}$ that this phenomenon actually occurs. To prove that fact we need firstly some preparation. The next result generalises Lemma5

Lemma 10. [4. Theorem C] Let $G$ be a $\pi$-separable group. If $\left|x^{G}\right|$ is a $\pi$-number for some $x \in G$, then $\left(\left\langle x^{G}\right\rangle\right)^{\prime}$ is a $\pi$-group. In particular, $x \in \mathbf{O}_{\pi, \pi^{\prime}}(G)$.

There are easy examples which illustrates that the above lemma is simply not true when the $\pi$-separability hypothesis is removed (cf. [4]).

The following well-known result is due to Itô. 
Lemma 11. [12, Proposition 5.1] Let $G$ be a group. Suppose that $p$ and $q$ are distinct primes that divide two different conjugacy class sizes of $G$, but there is no $g \in G$ with pq dividing $\left|g^{G}\right|$. Then $G$ is either p-nilpotent or q-nilpotent.

In relation to Theorem $B$, when we consider all the elements in the factors (not just those of order a $\pi$-number), we obtain the proposition below. Actually, this generalises [8. Lemma 6].

Proposition 3. Let $G=A B$ be the product of the subgroups $A$ and $B$, and assume that $G=$ $A B$ is both a core-factorisation and a class- $\pi$-separable factorisation. Then $G$ is $\pi$-separable.

Proof. Since $G=A B$ is a core-factorisation, there exists a chief series $1=N_{0} \unlhd N_{1} \unlhd \cdots \unlhd$ $N_{n-1} \unlhd N_{n}=G$ with each chief factor covered by either $A$ or $B$. In fact, we can refine that series in order to get a composition series whose factors are covered by either $A$ or $B$. Thus, for each $1 \leq i \leq n$, there exist subgroups $T_{j}$ such that $N_{i-1}=T_{0} \unlhd T_{1} \unlhd T_{2} \unlhd \cdots \unlhd T_{m}=N_{i}$ and $T_{j} / T_{j-1}$ is simple for every $1 \leq j \leq m$. We claim that each of these $T_{j} / T_{j-1}$ is either a $\pi$-group or a $\pi^{\prime}$-group, and so $G$ will be $\pi$-separable. Note that $T_{j} / T_{j-1}$ is isomorphic to $\left(T_{j} / N_{i-1}\right) /\left(T_{j-1} / N_{i-1}\right)$. Moreover $T_{j} / N_{i-1}$ is subnormal in $N_{i} / N_{i-1}$, which is normal in $G / N_{i-1}$ and it is covered by either $A$ or $B$, as $G / N_{i-1}=\left(A N_{i-1} / N_{i-1}\right)\left(B N_{i-1} / N_{i-1}\right)$ is a core-factorisation. Then all the class sizes of $N_{i} / N_{i-1}$, and so all the class sizes of $T_{j} / T_{j-1}$, are either $\pi$-numbers or $\pi^{\prime}$-numbers. If there are two primes $p \in \pi$ and $q \in \pi^{\prime}$ that divide two different class sizes of $T_{j} / T_{j-1}$, as $p q$ does not divide any class size of $T_{j} / T_{j-1}$, applying Lemma 11 we get that the simple group $T_{j} / T_{j-1}$ has either a normal $p$-complement or a normal $q$-complement. We deduce that either $p$ or $q$ does not divide the order of $T_{j} / T_{j-1}$, a contradiction. Thus, we may assume that each prime $q \in \pi^{\prime}$ does not divide any class size of $T_{j} / T_{j-1}$, so it has a central Sylow $q$-subgroup. It follows that $q \notin \pi\left(T_{j} / T_{j-1}\right)$ for every $q \in \pi^{\prime}$, so $T_{j} / T_{j-1}$ is a $\pi$-group and $G$ is $\pi$-separable.

We are now ready to prove Theorem $D$. We will use mainly Theorem $B$ and some of Dolfi's techniques in $[\overline{8}]$.

Theorem D. Let $G=A B$ be the product of the subgroups $A$ and $B$, and assume that $G=A B$ is both a core-factorisation and a class- $\pi$-separable factorisation. Then, up to abelian direct factors, one of the following two possibilities holds for any $X \in\{A, B\}$ :

(1) $X$ is either a $\pi$-group or a $\pi^{\prime}$-group.

(2) Up to interchanging $\pi$ and $\pi^{\prime}$, it holds $X=X_{\pi} X_{\pi^{\prime}}$ where $X_{\pi} \in \operatorname{Hall}_{\pi}(X)$ and $X_{\pi^{\prime}} \in$ $\operatorname{Hall}_{\pi^{\prime}}(X), X_{\pi^{\prime}} \unlhd X$, both $X_{\pi}$ and $X_{\pi^{\prime}}$ are abelian, and $X / \mathbf{O}_{\pi}(X)$ is a Frobenius group. Indeed, $\mathbf{O}_{\pi}(X)=\mathbf{Z}(X)$, the class sizes of $X$ are $\left\{1,\left|X_{\pi} / \mathbf{O}_{\pi}(X)\right|,\left|X_{\pi^{\prime}}\right|\right\}$, and $X$ is soluble.

In particular, both $A$ and $B$ are class- $\pi$-separable groups.

Proof. Observe that $G$ is $\pi$-separable by Proposition 3 . Take $H=(H \cap A)(H \cap B) \in \operatorname{Hall}_{\pi}(G)$ and $F=(F \cap A)(F \cap B) \in \operatorname{Hall}_{\pi^{\prime}}(G)$ with $H \cap X \in \operatorname{Hall}_{\pi}(X)$ and $F \cap X \in \operatorname{Hall}_{\pi^{\prime}}(X)$ for all $X \in\{A, B\}$ as in Lemma 3. Set $X_{\pi}:=H \cap X$ and $X_{\pi^{\prime}}:=F \cap X$. Certainly, $X=X_{\pi} X_{\pi^{\prime}}$. Let us analyse the structure of any $X \in\{A, B\}$. We may assume that $X$ has no abelian direct factors. We proceed in five steps.

STEP 1: Let $\sigma \in\left\{\pi, \pi^{\prime}\right\}$. If every $G$-class size of elements in $X$ is a $\sigma$-number, then $X$ is a $\sigma$-group.

Applying Theorem $\mathrm{B}$ for the elements in $X_{\sigma}$ and in $X_{\sigma^{\prime}}$, we deduce $X=X_{\sigma} \times X_{\sigma^{\prime}}$ with $X_{\sigma^{\prime}}$ abelian. Then $X_{\sigma^{\prime}}$ is an abelian direct factor of $X$, so $X_{\sigma^{\prime}}=1$ and $X$ is a $\sigma$-group, as wanted. 
In particular, we may assume in the sequel that $X$ is nor a $\pi$-group nor a $\pi^{\prime}$-group, and that there exist $x, y \in X$ such that $\pi\left(\left|x^{G}\right|\right)$ contains a prime in $\pi$ and $\pi\left(\left|y^{G}\right|\right)$ contains a prime in $\pi^{\prime}$, respectively.

STEP 2: $X$ has both abelian Hall $\pi$-subgroups and Hall $\pi^{\prime}$-subgroups.

In virtue of Theorem $\mathrm{B}$, we get that either every element in $X_{\pi}$ has $G$-class size a $\pi$-number or every element in $X_{\pi}$ has $G$-class size a $\pi^{\prime}$-number. In the first case we get $X=X_{\pi} \times X_{\pi^{\prime}}$ and, as we are assuming that $X_{\pi^{\prime}} \neq 1$, it cannot be an abelian direct factor, so necessarily there is a non-trivial element $y \in X_{\pi^{\prime}}$ with $\left|y^{G}\right|$ a $\pi^{\prime}$-number. Hence, for any $x \in X_{\pi} \backslash \mathbf{Z}\left(X_{\pi}\right)$ we get that $\left|(x y)^{G}\right|$ is neither a $\pi$-number nor a $\pi^{\prime}$-number, a contradiction. Hence all $\left|x^{G}\right|$ are $\pi^{\prime}$-numbers for the elements $x \in X_{\pi}$ and analogously all $\left|y^{G}\right|$ are $\pi$-numbers for the elements $y \in X_{\pi^{\prime}}$. Now Theorem $\mathrm{B}$ (a) yields that $X$ has abelian Hall $\pi$-subgroups and Hall $\pi^{\prime}$-subgroups, as wanted.

Note that our class size assumptions imply $X=\mathbf{C}_{X}\left(\mathbf{O}_{\pi}(G)\right) \cup \mathbf{C}_{X}\left(\mathbf{O}_{\pi^{\prime}}(G)\right)$, so we may assume $\left[X, \mathbf{O}_{\pi}(G)\right]=1$ in the remainder of the proof.

STEP 3: $X_{\pi^{\prime}}$ is normal in $X$. In particular, $X$ is soluble.

Denoting $\bar{G}:=G / \mathbf{O}_{\pi}(G)$, in virtue of Lemma 5 and Lemma 10 it follows $\overline{X_{\pi^{\prime}}} \leqslant \mathbf{O}_{\pi^{\prime}}(\bar{G}) \cap$ $\bar{X} \leqslant \mathbf{O}_{\pi^{\prime}}(\bar{X})$. Since $X_{\pi^{\prime}} \in \operatorname{Hall}_{\pi^{\prime}}(X)$, we get $\overline{X_{\pi^{\prime}}}=\mathbf{O}_{\pi^{\prime}}(\bar{X})$, and then $X_{\pi^{\prime}} \mathbf{O}_{\pi}(G)$ is normalised by $X$. Now for any $x \in X$, we deduce that $X_{\pi^{\prime}}^{x} \leqslant\left(X_{\pi^{\prime}} \mathbf{O}_{\pi}(G)\right)^{x}=X_{\pi^{\prime}} \mathbf{O}_{\pi}(G)$, so there exists some $n \in \mathbf{O}_{\pi}(G)$ such that $X_{\pi^{\prime}}^{x}=X_{\pi^{\prime}}^{n}$. As $\left[X, \mathbf{O}_{\pi}(G)\right]=1$, then $X_{\pi^{\prime}} \unlhd X$, and $X$ is soluble.

STEP 4: $\mathbf{O}_{\pi}(X)=\mathbf{Z}(X)$.

Since $X_{\pi^{\prime}}$ is abelian and normal in $X$, by coprime action, we deduce $X_{\pi^{\prime}}=\left[X_{\pi^{\prime}}, X_{\pi}\right] \times$ $\mathbf{C}_{X_{\pi^{\prime}}}\left(X_{\pi}\right)$. Note that $\mathbf{C}_{X_{\pi^{\prime}}}\left(X_{\pi}\right) \leqslant \mathbf{Z}(X)$, so $\mathbf{C}_{X_{\pi^{\prime}}}\left(X_{\pi}\right)$ is an abelian direct factor of $X$ and we may assume $\mathbf{C}_{X_{\pi^{\prime}}}\left(X_{\pi}\right)=1$. Hence $X_{\pi^{\prime}} \cap \mathbf{Z}(X)=1$ and $\mathbf{Z}(X) \leqslant \mathbf{O}_{\pi}(X)$. The other inclusion is clear because $X_{\pi^{\prime}}$ is normal in $X$ and $X_{\pi}$ is abelian.

STEP 5: $X / \mathrm{O}_{\pi}(X)$ is a Frobenius group. In particular, the set of class sizes of $X$ is $\left\{1,\left|X_{\pi} / \mathbf{O}_{\pi}(X)\right|,\left|X_{\pi^{\prime}}\right|\right\}$.

Set $\tilde{X}:=X / \mathbf{O}_{\pi}(X)$. We claim that $\tilde{X}_{\pi}$ acts fixed-point-freely on $\tilde{X_{\pi^{\prime}}}$. Take $1 \neq \tilde{y} \in \tilde{X_{\pi^{\prime}}}$ and $\tilde{x} \in \tilde{X}_{\pi}$ such that $[\tilde{y}, \tilde{x}]=1$. Then $[y, x] \in \mathbf{O}_{\pi}(X)$ and it is a $\pi^{\prime}$-element since $X_{\pi^{\prime}} \unlhd X$. Now $x y=y x$ and both $\left|x^{G}\right|$ and $1 \neq\left|y^{G}\right|$ divides $\left|(x y)^{G}\right|$. It follows necessarily that $x \in$ $\mathbf{Z}(G) \cap X \leqslant \mathbf{Z}(X)=\mathbf{O}_{\pi}(X)$ so $\tilde{x}=1$ and we are done.

Finally, note that $\mathbf{O}_{\pi}(X)=\mathbf{Z}(X)$ implies $\mathbf{Z}(X) \cap X^{\prime} \leqslant \mathbf{Z}(X) \cap X_{\pi^{\prime}}=1$. This fact leads to $\mathbf{C}_{X}(g) / \mathbf{Z}(X)=\mathbf{C}_{X / \mathbf{Z}(X)}(g \mathbf{Z}(X))$ for all $g \in X$, so the class sizes of $X$ and $X / \mathbf{Z}(X)$ coincides. Since $X / \mathbf{Z}(X)$ is a Frobenius group, then clearly the set of class sizes of $X$ is $\left\{1,\left|X_{\pi} / \mathbf{O}_{\pi}(X)\right|,\left|X_{\pi^{\prime}}\right|\right\}$.

To conclude, from the described structure of $X$, we get that $X$ is a class- $\pi$-separable group.

Observe that [8, Theorem 4] (Theorem 2 above) is now a direct consequence of the previous theorem when $G=A=B$.

Example 7. A core-factorisation whose factors are two class- $\pi$-separable groups might not be a class- $\pi$-separable factorisation: Let $G$ be the semidirect product of a cyclic group of order 35 and a cyclic group of order 6. If we take $\pi:=\{3,7\}, A \in \operatorname{Hall}_{\pi}(G)$ and $B \in \operatorname{Hall}_{\pi^{\prime}}(G)$, then $G=A B$ is a core-factorisation since $G$ is $\pi$-separable (indeed it is soluble). Certainly, $G=A B$ is not a class- $\pi$-separable factorisation, although $A$ and $B$ are class- $\pi$-separable groups.

The next example illustrates that, in a class- $\pi$-separable factorisation, $G$ might be nonsoluble, and both the Hall $\pi$-subgroups and the Hall $\pi^{\prime}$-subgroups of $G$ might be non-abelian. 
Example 8. Let $A$ be an alternating group of degree 5 and $B$ a Frobenius group of order $29 \cdot 7$. Consider $G=A \times B$, and $\pi=\{2,3,5\}$. Clearly, every class size of an element in $A \cup B$ is either a $\pi$-number or a $\pi^{\prime}$-number, but $G$ is non-soluble. Moreover, neither the Hall $\pi$-subgroup nor the Hall $\pi^{\prime}$-subgroup of $G$ is abelian.

To conclude, inspired by [4], we concentrate on factorised groups whose $\pi$-elements in the factors have conjugacy class lengths equal to prime powers. Indeed, in [10] we analysed products of groups where, for a given prime $p$, the $p$-elements in the factors have prime power class sizes.

Proposition 4. [10. Theorems A and B] Let $G=A B$ be the product of the subgroups $A$ and $B$, and let $P \in \operatorname{Syl}_{p}(G)$. Assume that $\left|g^{G}\right|$ is equal to a prime power for each p-element $g \in A \cup B$. Then we have:

(1) $P \mathbf{F}(G)$ is normal in $G$.

(2) There exist unique primes $q$ and $r$ such that $\left|x^{G}\right|$ is a q-number for every $p$-element $x \in A$, and $\left|y^{G}\right|$ is an $r$-number for every $p$-element $y \in B$, respectively. (Possibly, $p \in\{q, r\}$ or $q=r$.)

Lemma 12. Let $G$ be a group, and let $x, y \in G \backslash \mathbf{Z}(G)$ be $\pi$-elements such that $\left|x^{G}\right|$ and $\left|y^{G}\right|$ are two distinct prime powers, and assume that $\left|(x y)^{G}\right|$ is also a prime power. Then $\langle x, y\rangle^{G} \leqslant \mathbf{O}_{\pi}(G)$, and $\left|(x y)^{G}\right|=\max \left\{\left|x^{G}\right|,\left|y^{G}\right|\right\}$ is a power of a prime $q \in \pi$. In particular, if $\operatorname{Hall}_{\pi}(G) \neq \emptyset$, then a Hall $\pi$-subgroup of $G$ is non-abelian.

Proof. It is enough to mimic the proof of [6. Lemma 4] with $\pi$ instead of $p$.

Theorem E. Let $G=A B$ be a core-factorisation. Suppose that $\left|x^{G}\right|$ is a prime power for every $\pi$-element $x \in A \cup B$. Then $G$ is $\pi$-separable of $\pi$-length at most 1 . Moreover, for each $X \in\{A, B\}$, one of the following two possibilities holds:

(1) All $\left|x^{G}\right|$ are powers of a fixed prime $q$ for every $\pi$-element $x \in X$. In addition:

(a) If $q \notin \pi$, then $X$ has an abelian Hall $\pi$-subgroup $X_{\pi}$. In this case $X_{\pi} \mathbf{O}_{q}(G)$ is normalised by $X$.

(b) If $q \in \pi$, then $X$ is $\pi$-decomposable with nilpotent Hall $\pi$-subgroup $X_{\pi}$ and the Sylow subgroups of $X_{\pi}$ are all abelian except possibly for the prime $q$.

(2) All $\left|x^{G}\right|$ are powers of two distinct fixed primes $q$ and $r$, both in $\pi$, for every $\pi$-element $x \in X$. In this case, $X$ is $\pi$-decomposable, and the Hall $\pi$-subgroup $X_{\pi}$ of $X$ satisfies that $X_{\pi} / \mathbf{Z}\left(X_{\pi}\right)$ is a Frobenius group with abelian kernel and complement of orders a $q$-power and an r-power, respectively.

Proof. First of all, we prove the assertion on the $\pi$-separability of $G$. Applying Proposition 4 (1) for each prime $p \in \pi$, we can affirm that $G / \mathbf{F}(G)$ has a normal Sylow $p$-subgroup. Therefore, $G$ is $p$-separable with $p$-length at most 1 for each prime $p \in \pi$ and, in particular, it is $\pi$-separable with $\pi$-length at most 1 . Henceforth, we can take $H=(H \cap A)(H \cap B) \in \operatorname{Hall}_{\pi}(G)$ with $H \cap A \in \operatorname{Hall}_{\pi}(A)$ and $H \cap B \in \operatorname{Hall}_{\pi}(B)$.

Next we assume that every $\left|x^{G}\right|$ is a power of a fixed prime $q$ for the elements in $H \cap X$, as in case (1). If $q \notin \pi$, then we obtain that $X$ has an abelian Hall $\pi$-subgroup $X_{\pi}:=H \cap X$ in virtue of Theorem $\mathrm{B}$ (a). Let us prove that $X_{\pi} \mathbf{O}_{q}(G)$ is normalised by $X$. Since $G / \mathbf{F}(G)$ has a normal Sylow $p$-subgroup for each $p \in \pi$, then $H \mathbf{F}(G)=H \mathbf{O}_{\pi^{\prime}}(\mathbf{F}(G)) \unlhd G$. We denote by bars the quotients over $\mathbf{O}_{\pi^{\prime}}(\mathbf{F}(G))$, so $\bar{H}=\mathbf{O}_{\pi}(\bar{G})$. In particular $\overline{X_{\pi}} \leqslant \mathbf{O}_{\pi}(\bar{G}) \cap \bar{X} \leqslant \mathbf{O}_{\pi}(\bar{X})$, and then $X_{\pi} \mathbf{O}_{\pi^{\prime}}(\mathbf{F}(G))$ is normal in $X \mathbf{O}_{\pi^{\prime}}(\mathbf{F}(G))$. Since $\left|x^{G}\right|$ is a $q$-power for all the elements in $X_{\pi}$, then $\mathbf{O}_{q^{\prime}}(\mathbf{F}(G))$ centralises $X_{\pi}$. Thus $X_{\pi} \mathbf{O}_{q}(G)$ is a normal Hall $\pi \cup\{q\}$-subgroup of $X_{\pi} \mathbf{O}_{\pi^{\prime}}(\mathbf{F}(G))$, so $X_{\pi} \mathbf{O}_{q}(G)$ is normal in $X \mathbf{O}_{\pi^{\prime}}(\mathbf{F}(G))$. Hence $X_{\pi} \mathbf{O}_{q}(G)$ is normalised by $X$, as wanted in (a). 
If $q \in \pi$, then Theorem $\mathrm{B}$ (b) provides that $X=X_{\pi} \times \mathbf{O}_{\pi^{\prime}}(X)$, so it remains to show that $X_{\pi}$ is nilpotent with abelian Sylow subgroups, except possibly for the prime $q$. Recall that $G$ is $p$-separable for every prime $p \in \pi$. Hence, Theorem $\mathrm{B}$ (b) applied for the prime $q$ gives $X=X_{q} \times \mathbf{O}_{q^{\prime}}(X)$, so $X_{\pi}=X_{q} \times \mathbf{O}_{\sigma}(X)$ where $\sigma:=\pi \backslash\{q\}$. Finally, $\mathbf{O}_{\sigma}(X)$ is abelian in virtue again of Theorem $\mathrm{B}$ (a) applied for $\sigma$, and (b) is proved.

From now on, we assume that, for either $X=A$ or $X=B$, there exist $x_{1}, x_{2} \pi$-elements in $X$ such that $\left|x_{1}^{G}\right|$ and $\left|x_{2}^{G}\right|$ are powers of distinct primes.

STEP 1: At most two different primes appear as divisors of the class sizes of the $\pi$-elements in $X$.

Assuming the contrary, there exists three non-central $\pi$-elements in $X$, say $x_{1}, x_{2}$ and $x_{3}$, such that their $G$-class sizes are equal to powers of three different primes, say $p_{1}, p_{2}$ and $p_{3}$, respectively. All the $x_{i}$ decompose as product of commuting prime power order $(\pi$-)elements, so Proposition 4 (2) joint with this last fact allow us to suppose that the orders of the $x_{i}$ are coprime prime powers. Hence, from now on we assume that $x_{i}$ is a $q_{i}$-element with $\left|x_{i}^{G}\right|$ equal to a $p_{i}$-power, for each $i \in\{1,2,3\}$. Since either $p_{1} \neq q_{2}$ or $p_{1} \neq q_{3}$, we may assume the first case and so there exists $g \in G$ such that $Q^{g} \leqslant \mathbf{C}_{G}\left(x_{1}\right)$, where $Q=(Q \cap A)(Q \cap B) \in \operatorname{Syl}_{q_{2}}(G)$, $Q \cap A \in \operatorname{Syl}_{q_{2}}(A)$ and $Q \cap B \in \operatorname{Syl}_{q_{2}}(B)$. We may suppose $x_{2} \in Q \cap X$ by Remark 2 , and so we get $x_{2}^{g} \in \mathbf{C}_{G}\left(x_{1}\right)$. But $\left(\left|x_{1}^{G}\right|,\left|x_{2}^{G}\right|\right)=1$, so $G=\mathbf{C}_{G}\left(x_{1}\right) \mathbf{C}_{G}\left(x_{2}\right)$ and we obtain $x_{2} \in \mathbf{C}_{G}\left(x_{1}\right)$. Now $x_{1} x_{2}=x_{2} x_{1} \in X$ is a $\pi$-element, and it follows that $\left|\left(x_{1} x_{2}\right)^{G}\right|$ is divisible by both $p_{1}$ and $p_{2}$, a contradiction.

STEP 2: Assuming that all $\left|x^{G}\right|$ are powers of two distinct fixed primes $q$ and $r$ for every $\pi$-element $x \in X$, we claim that $\{q, r\} \subseteq \pi$.

Let $x$ and $y$ be $\pi$-elements in $X$ such that $\left|x^{G}\right|$ is a non-trivial $q$-power and $\left|y^{G}\right|$ is a nontrivial $r$-power. Again by Remark 2 , we can assume without loss of generality that $x, y \in H \cap X$. Hence $x y \in H \cap X$ and $\left|(x y)^{G}\right|$ is a prime power also. Thus, in virtue of Lemma 12 we have that the prime which corresponds to the largest class size lies in $\pi$. So let us suppose that the largest one is $\left|x^{G}\right|$, that is, $q \in \pi$. If $r \notin \pi$, then there exists $g \in G$ such that $x^{g} \in H^{g} \leqslant \mathbf{C}_{G}(y)$. Also $G=\mathbf{C}_{G}(x) \mathbf{C}_{G}(y)$, so $x y=y x \in H \cap X$ and we conclude that $\left|(x y)^{G}\right|$ is divisible by both $q$ and $r$, a contradiction.

STEP 3: $X$ is $\pi$-decomposable, and the Hall $\pi$-subgroup $X_{\pi}$ of $X$ satisfies that $X_{\pi} / \mathbf{Z}\left(X_{\pi}\right)$ is a Frobenius group with abelian kernel and complement of orders a $q$-power and an $r$-power, respectively.

Since we are assuming that all $\left|x^{G}\right|$ are powers of two distinct fixed primes $q$ and $r$, both in $\pi$, for every $\pi$-element $x \in X$, then by Theorem $\mathrm{B}$ we get that $X_{\pi}$ centralises every Hall $\pi^{\prime}$-subgroup of $G$. Indeed it is $\pi$-decomposable. For proving the remaining assertion, we distinguish two cases for the class sizes of the $\pi$-elements in $Y$, where $\{X, Y\}=\{A, B\}$ : either they are powers of a prime in $\pi$ or in $\pi^{\prime}$. In the second case, by Theorem $B$ we obtain $Y_{\pi}:=H \cap Y \leqslant \mathbf{Z}(H) \leqslant \mathbf{C}_{H}\left(X_{\pi}\right)$. As $X_{\pi}$ centralises every Hall $\pi^{\prime}$-subgroup of $G$, it follows that $X_{\pi}$ is normal in $G$. Then all the $X_{\pi}$-class sizes of elements in $X_{\pi}$ are either $q$-powers or $r$-powers, and Theorem 2 yields the desired structure of $X_{\pi} / \mathbf{Z}\left(X_{\pi}\right)$. In the other case, $Y_{\pi}$ also centralises every Hall $\pi^{\prime}$-subgroup of $G$, so $H=X_{\pi} Y_{\pi}$ is normal in $G$. We deduce that the class sizes in $H$ of all elements in $X_{\pi} \cup Y_{\pi}$ are either $q$-powers or $r$-powers. But we may affirm that $H=X_{\pi} X_{\pi^{\prime}}$ is a core-factorisation in virtue of Lemma 3, so Theorem Dapplied to $H$ completes the proof of (2).

The main result of [4] now can be retrieved from the above theorem (see the corollary below). It is significant to notice that our proof, however, uses different tools. 
Corollary 2. Let $G$ be a group for which every $\left|x^{G}\right|$ is a prime power for the $\pi$-element $x \in G$. Then one of the following possibilities occurs.

(1) All $\left|x^{G}\right|$ are powers of a fixed prime $q$. Moreover,

(a) $q \notin \pi$ if and only if $G$ has an abelian Hall $\pi$-subgroup $H$. In this case, $H \mathbf{O}_{q}(G)$ is normal in $G$.

(b) $q \in \pi$ if and only if $G$ is $\pi$-decomposable with nilpotent Hall $\pi$-subgroup $H$, and the Sylow subgroups of $H$ are all abelian except possibly for the prime $q$.

(2) All $\left|x^{G}\right|$ are powers of two distinct primes, say $q$ and $r$. This happens if and only if $\{q, r\} \subseteq \pi, G$ is $\pi$-decomposable, and the Hall $\pi$-subgroup $H$ of $G$ satisfies that $H / \mathbf{Z}(H)$ is a Frobenius group with abelian kernel and complement of orders a q-power and an $r$ power, respectively.

Furthermore, in all cases, $G$ has $\pi$-length at most 1.

The following example gives insight into the possible global $\pi$-structure of a group satisfying the hypotheses of Theorem E

Example 9. Let $A$ be a symmetric group of degree 3 and $B$ a dihedral group of order 10 . Consider $G=A \times B$, and $\pi=\{2,3\}$. Clearly, the hypotheses in Theorem E are satisfied, but neither the Hall $\pi$-subgroup of $G$ is abelian (as in case (1)(a) above) nor $G$ is $\pi$-decomposable (case (2) above).

\section{References}

[1] B. Amberg, S. Franciosi, And F. de Giovanni. Products of groups. Oxford University Press Inc., 1992.

[2] A. Ballester-Bolinches, J. Cossey, And Y. Li. "Mutually permutable products and conjugacy classes". Monatsh. Math. 170 (2013) 305-310.

[3] A. Ballester-Bolinches, R. Esteban-Romero, And M. As AAD. Products of finite groups. Vol. 53. W. de Gruyter Expositions In Mathematics, 2010.

[4] A. Beltrán AND M. J. FeliPe. "Prime powers as conjugacy class lengths of $\pi$-elements". Bull. Austral. Math. Soc. 69 (2004) 317-325.

[5] A. Beltrán, M. J. Felipe, G. Malle, A. Moretó, G. Navarro, L. Sanus, R. Solomon, AND P. H. TIEP. "Nilpotent and abelian Hall subgroups in finite groups". Trans. Amer. Math. Soc. 368 (2016) 2497-2513.

[6] Y. BERKOVICH AND L. S. KAZARIN. "Indices of elements and normal structure of finite groups". J. Algebra 283 (2005) 564-583.

[7] K. Doerk And T. Hawkes. Finite Soluble Groups. Vol. 4. W. de Gruyter Expositions In Mathematics, 1992.

[8] S. DoLfI. "Arithmetical conditions on the length of the conjugacy classes of a finite group". $J$. Algebra 174 (1995) 753-771.

[9] S. Dolfi, E. Pacifici, L. SAnus, AND P. Spiga. "On the orders of zeros of irreducible characters". J. Algebra 321 (2009) 345-352.

[10] M. J. Felipe, A. Martínez-Pastor, And V. M. Ortiz-Sotomayor. "Prime power indices in factorised groups". Mediterr. J. Math. 14.6 (2017) article: 225.

[11] M. J. Felipe, A. Martínez-Pastor, And V. M. Ortiz-Sotomayor. "Zeros of irreducible characters in factorised groups". Ann. Mat. Pura Appl. 198 (2019) 129-142.

[12] N. ITÔ. "On finite groups with given conjugate types, I.” Nagoya Math. J. 6 (1953) 17-28.

[13] G. NAVARro AND P.H. TIEP. “Abelian Sylow subgroups in a finite group”. J. Algebra 398 (2014) 519-526. 
[14] X. H. Zhao, X. Y. Guo, AND J. Y. ShI. "On the conjugacy class sizes of prime power order $\pi$-elements”. Southeast Asian Bull. Math. 35 (2011) 735-740. 
